Fine ergodic properties of partially hyperbolic dynamical systems 

SERVIÇO DE PÓS-GRADUAÇÃO DO ICMC-USP

Data de Depósito:

Assinatura:

\title{
Fine ergodic properties of partially hyperbolic dynamical systems
}

\author{
Gabriel Ponce
}

Advisor: Prof. Dr. Ali Tahzibi

Doctoral dissertation submitted to the Instituto de Ciências Matemáticas e de Computação - ICMC-USP, in partial fulfillment of the requirements for the degree of the Doctorate Program in Mathematics. FINAL VERSION. 
Ficha catalográfica elaborada pela Biblioteca Prof. Achille Bassi e Seção Técnica de Informática, ICMC/USP, com os dados fornecidos pelo(a) autor(a)

\begin{tabular}{|c|c|}
\hline \multirow[t]{3}{*}{ P792f } & $\begin{array}{l}\text { Ponce, Gabriel } \\
\text { Fine ergodic properties of partially hyperbolic } \\
\text { dynamical systems / Gabriel Ponce; orientador Ali } \\
\text { Tahzibi. -- São Carlos, } 2014 \text {. } \\
\quad 92 \text { p. }\end{array}$ \\
\hline & $\begin{array}{l}\text { Tese (Doutorado - Programa de Pós-Graduação em } \\
\text { Matemática) -- Instituto de Ciências Matemáticas e } \\
\text { de Computação, Universidade de São Paulo, } 2014 .\end{array}$ \\
\hline & $\begin{array}{l}\text { 1. Dynamical Systems. 2. Partially hyperbolic } \\
\text { diffeomorphisms. 3. Disintegration of measures. } 4 \text {. } \\
\text { Foliations. 5. Ergodic theory. I. Tahzibi, Ali, } \\
\text { orient. II. Título. }\end{array}$ \\
\hline
\end{tabular}


SERVIÇO DE PÓS-GRADUAÇ̃̃O DO ICMC-USP

Data de Depósito:

Assinatura:

\title{
Propriedades ergódicas finas de sistemas dinâmicos parcialmente hiperbólicos
}

\author{
Gabriel Ponce
}

Orientador: Prof. Dr. Ali Tahzibi

\begin{abstract}
Dissertação apresentada ao Instituto de Ciências Matemáticas e de Computação - ICMC-USP, como parte dos requisitos para obtenção do título de Doutor em Ciências - Matemática. VERSÃO FINAL.
\end{abstract}



A Deus,

aos meus pais Ana e Helio

e aos meus irmãos Pedro, Davi e Tiago. 



\section{Agradecimentos}

Esta tese significa a conclusão de uma longa jornada que se iniciou ainda no meu ensino médio. Ao longo de todos esses anos foram muitas as pessoas que me incentivaram e que contribuíram para que essa conquista fosse possível.

Agradeço primeiramente a Deus, que durante todo o trajeto foi minha fortaleza e suporte, guiando meus passos e decisões e sendo mais doce a cada dia.

O vetor inicial da minha motivação na matemáfica foi dado pelos meus professores de ensino médio, especialmente Eloy Machado e Paulo Vita, e pelo Collegium Sapiens onde cursei o ensino médio. Agradeço ao Collegium Sapiens e ao seu corpo docente, pois esse incentivo influenciou toda a minha carreira e me fez escolher a profissão que exerço com tanta satisfação.

Ainda no ensino médio conheci o professor Ali Tahzibi, o qual tem sido para mim como um tutor em matemática, desde a graduação. Sempre com entusiasmo ímpar e uma motivação infinita pela pesquisa, o professor Ali foi o responsável por grande parte da minha formação e pela decisão de pesquisar em Sistemas Dinâmicos. Agradeço ao professor Ali Tahzibi pelos anos de dedicação, pela paciência e pela amizade.

Ao longo da graduação, muitos professores foram de suma importância na minha formação. É impossível citar todos os nomes, mas aqui destaco as professoras Sandra Godoy e Maria Ruas, às quais nos meus primeiros anos de graduação, motivaram-me e ajudaram-me de diversas formas a pensar objetivos elevados e a obter maturidade na carreira. 
Agradeço a todo corpo docente do ICMC pela formação proporcionada e por todo o apoio que me deram durante minha graduação e pós-graduação.

Agradeco ao meu supervisor no exterior Federico Hertz, ao corpo docente e aos alunos de doutorado da Penn State University pela hospitalidade e atenção durante meu estágio de doutorado no exterior.

Agradeço também a todos os meus amigos, de graduação e pós-graduação, no ICMC pelos anos de aprendizado, companhia e apoio. Agradeço em particular ao Régis Varão pelos trabalhos em colaboração e pela ajuda na correção dessa tese.

Agradeço à FAPESP pelo suporte financeiro concedido, o qual tornou possível a realização deste projeto de doutorado, bem como o estágio realizado no exterior.

Agradeço aos meus irmãos Lindomar e Adriana e a todos os meus irmãos na fé pelo incentivo, força e palavras de encorajamento durante o meu doutorado, especialmente durante meu estágio no exterior.

Agradeco à minha namorada Júlia, que esteve ao meu lado nos momentos de alegria e frustrações, sempre transmitindo ânimo e confiança em cada decisão.

Agradeço aos meus parentes, especialmente às minhas tias Ivani e Rose, que sempre me apoiaram na escolha deste curso e me motivaram a seguir a carreira acadêmica.

Finalmente, agradeço a minha família, meus pais Ana Ponce e Helio Ponce, e meus irmãos, Pedro, Davi e Tiago por sempre acreditarem em mim. Eles são aqueles que estão comigo desde antes de eu aprender as operações mais básicas e compartilharam comigo de todas as circunstâncias dessa jornada. Eles estiveram comigo em todos os vales e montanhas e ajudaram a moldar meu caráter e ética de trabalho. Apesar de não serem matemáticos, foi com eles que aprendi as lições mais importantes da minha vida. 


\section{Resumo}

Seja $f: \mathbb{T}^{3} \rightarrow \mathbb{T}^{3}$ um difeomorfismo $C^{2}$ parcialmente hiperbólico, homotópico a um automorfismo de Anosov linear e preservando a medida de volume $m$. Provamos que se $f$ é Kolmogorov então $f$ é Bernoulli.

Estudamos as características da desintegração atômica da medida de volume quando esta ocorre. Provamos que se a medida de volume $m$ tem desintegração atômica nas folhas centrais então a desintegração tem um átomo por folha central. Apresentamos uma condição, a qual depende apenas do expoente de Lyapunov central do difeomorfismo, que garante desintegração atômica da medida de volume. Construímos uma família aberta de difeomorfismos satisfazendo esta condição, o que gerou os primeiros exemplos de folheações que são mensuráveis e ao mesmo tempo minimais. Nesta mesma construção damos os primeiros exemplos de difeomorfismos parcialmente hiperbólicos com expoente de Lyapunov central nulo e homotópico a um Anosov linear. 


\section{Abstract}

Let $f: \mathbb{T}^{3} \rightarrow \mathbb{T}^{3}$ be a $C^{2}$ volume preserving partially hyperbolic diffeomorphism homotopic to a linear Anosov automorphism $A: \mathbb{T}^{3} \rightarrow \mathbb{T}^{3}$. We prove that if $f$ is Kolmogorov, then $f$ is Bernoulli.

We study the characteristics of atomic disintegration of the volume measure whenever it occurs. We prove that if the volume measure $m$ has atomic disintegration on the center leaves then the disintegration has one atom per center leaf. We give a condition, depending only on the center Lyapunov exponent of the diffeomorphism, that guarantees atomic disintegration of the volume measure on center leaves. We construct an open family of diffeomorphisms satisfying this condition which generates the first examples of foliations which are both measurable and minimal. In this same construction we give the first examples of partially hyperbolic diffeomorphisms with zero center Lyapunov exponent and homotopic to a linear Anosov. 


\section{Contents}

1 Introduction $\quad 1$

1.1 Equivalence of Kolmogorov and Bernoulli property . . . . . . . . . . 4

1.2 Disintegration of volume measure and pathological examples . . . . . 6

1.3 Structure of the thesis . . . . . . . . . . . . . . . . . 8

2 Preliminaries $\quad 9$

2.1 Partially Hyperbolic Dynamics . . . . . . . . . . . . . . . . . . . . . 9

2.1.1 Franks-Manning semi-conjugacy . . . . . . . . . . . . 13

2.2 Measurable partitions and disintegration of measures . . . . . . . . 14

2.3 Ergodic Hierarchy . . . . . . . . . . . . . . . . . . . . . . . . . 18

2.3.1 Kolmogorov property and partial hyperbolicity . . . . . . . 21

3 Pathological Dynamics $\quad 27$

3.1 Local Perturbation . . . . . . . . . . . . . . . . . . . . . . 29

3.2 Family of Linear Anosov Automorphisms . . . . . . . . . . . . . . . . 31

3.3 Proof of Theorems C and E . . . . . . . . . . . . . . . 32

3.3.1 Size of perturbation among absolutely partially hyperbolic diffeomorphisms . . . . . . . . . . . . . 36

3.4 Ergodicity of the pathological example . . . . . . . . . . . . 42

4 Atomic disintegration for DA diffeomorphisms 45

4.1 Disintegration of volume along invariant foliations . . . . . . . . . . . 47

4.1.1 Disintegration of volume along center foliation . . . . . . . . . 48

4.1.2 Comments on the results of this chapter . . . . . . . . . . 49 
4.2 Proof of Theorem B . . . . . . . . . . . . . . . . 50 50

4.3 A Glimpse of Pesin Theory . . . . . . . . . . . . . . . . . . . 55

4.4 Proof of Theorems D and $\mathrm{F} \ldots \ldots \ldots \ldots 6$

5 Bernoulli property for DA diffeomorphisms on $\mathbb{T}^{3} \quad 61$

5.1 The Very Weak Benoulli property . . . . . . . . . . . . . . . 64

5.2 Kolmogorov property . . . . . . . . . . . . . 66

5.3 Proof of Theorem A . . . . . . . . . . . . . . . . 67

5.3.1 Partition by rectangles . . . . . . . . . . . . . 70

5.3.2 Construction of the function $\theta \ldots \ldots$. . . . . . 76

5.3.3 Conclusion of the proof of Theorem A . . . . . . . . . . . 83

$\begin{array}{lr}\text { Bibliography } & 87\end{array}$ 


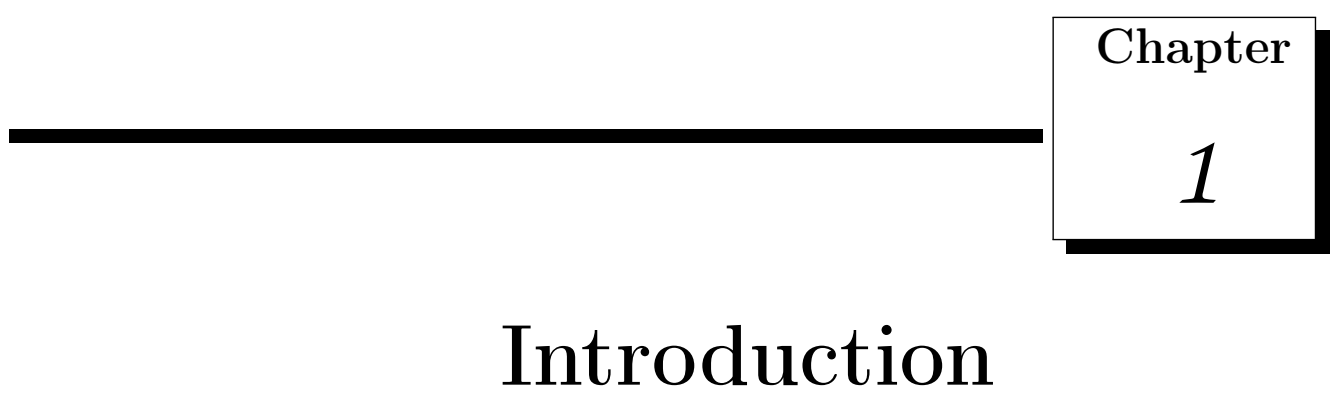

Given an ambient manifold $M$ and a function $f: M \rightarrow M$, the concept of the dynamics of $f$ may be seem as the behavior of the orbit of a point $x$ through $f$. This "behavior" can be analyzed and classified from several different aspects and angles. We can classify and distinguish dynamics of a system by algebraic properties, topological properties, metric properties, etc. One example of distinguishing dynamics by topological properties of its orbits is the concept of minimality of an orbit. Given a point $x$ what happens with the distribution of $\left\{f^{n}(x)\right\}_{n}$ ? Is it minimal, i.e, dense on the manifold $M$ ?

Another way of classifying dynamics is through statistical and geometric properties of invariant measures of systems in measurable spaces. The study of such properties constitutes the ergodic theory of dynamical systems. Consider an ambient manifold $M$ endowed with a probability measure $\mu$, and a function $f: M \rightarrow M$ invariant by the measure $\mu$, that is, $\mu\left(f^{-1}(A)\right)=\mu(A)$ for all measurable set $A$. We can analyze how unpredictable is this system from the point of view of the measure by saying that the only sets from which the orbits never scapes, have measure zero or one. This property is called ergodic property of the system.

Although ergodicity is a form of saying that the system is unpredictable from the point of view of the measure, we may find several "degrees of unpredictability". These different degrees of unpredictability constitute what we call ergodic hierarchy. Intuitively speaking the ergodic hierarchy distinguishes systems by how fast they mix sets along the time. Between those fine ergodic properties, we cite for example: 
Bernoulli property, Kolmogorov property, mixing, ergodicity.

The Bernoulli property is the strongest type of unpredictability in terms of measure, meaning that we can find a symbolic representation of the system equivalent to a shift, that is, we can find a finite partition of the system where the symbolic representation generated by this partition is measurably equivalent to a standard Bernoulli shift. If we look at this definition in terms of information of orbits, it tells us that with respect to this finite partition, even if you are given all the future and all the past information of an orbit you still cannot say to which element of the partition the initial point belongs! This is the extreme degree of unpredictability, or "chaos in terms of measure". Natural examples of Bernoulli automorphisms are the linear toral automorphisms without eigenvalues of norm one [61]. Mixing and ergodicity are also very well known properties. The Kolmogorov property is not as intuitive as the other properties but it is an extremely important property and can be described by the notion of entropy. Roughly speaking, an automorphism $f$, preserving a measure $\mu$, is Kolmogorov if for every non-trivial finite partition of the space the $\mu$-entropy of $f$ with respect to the given partition is positive, that is, every finite partition gives some information of the dynamics.

Once we have defined a hierarchy of ergodic properties and a concept of extreme unpredictability (the Bernoulli property), several natural and important questions arise. For example:

- How can we classify all the Bernoulli automorphisms?

- For which class of automorphisms the hierarchy is strict?

- What are the natural examples of transformations which are Bernoulli?

- What kind of structures gives rise to a Bernoullian dynamics?

Ergodic theory has two main strands: abstract ergodic theory and smooth ergodic theory.

Abstract ergodic theory studies intrinsic ergodic properties of automorphisms in a given measurable space taking into consideration measurable characteristics of the automorphism such as metric entropy and other invariants (here invariance is with respect to measurable isomorphism). On the other hand, smooth ergodic theory also studies the ergodic properties of systems for which we have some type of smooth structure. Thus, the basic setting of smooth ergodic theory is usually a 
diffeomorphism $f: M \rightarrow M$, where $M$ is a manifold, preserving a given measure $\mu$ on $M$.

While the first question (the classification of Bernoulli automorphisms) is a question inside the scope of abstract ergodic theory, the other three questions were developed using the intersection of the two strands of ergodic theory.

In the seventies D. Ornstein [38], [40] made a major contribution to abstract ergodic theory proving that Bernoulli automorphisms are completely classified by their entropy, answering the question of the first item. As cited before, Y. Katznelson [61] showed that linear ergodic automorphisms on tori are Bernoulli and in [14] Ornstein-Weiss proved that the geodesic flow on a negatively curved Riemannian surface is Bernoulli, and to do so they used the fact that the geodesic flow in this kind of surfaces are Kolmogorov. The smooth structure exhibited by these examples were fundamental to obtain Bernoulli property, what shows the importance of smooth ergodic theory inside abstract ergodic theory. This shows natural examples of Bernoulli automorphisms. In light of this result and the technique used by Ornstein-Weiss, it was clear that the presence of hyperbolic structures together with some degree of uncertainty (in this case this degree is given by the Kolmogorov property) implies extreme unpredictability, i.e, the Bernoulli property.

Both the ergodic linear automorphisms on tori and the time-1 map of the geodesic flow on negatively curved surfaces are examples of partially hyperbolic diffeomorphisms which is a concept more general than hyperbolic diffeomorphisms. A system is called absolutely partially hyperbolic if it has expanding and contracting directions, and a center direction where we may have some degree of expansion and contraction but dominated by the other directions. We denote by $\mathcal{P} \mathcal{H}_{\mu}^{r}(M)$ the set of $C^{r}$ absolutely partially hyperbolic diffeomorphisms of $M$ preserving the probability measure $\mu$. If an absolutely partially hyperbolic diffeomorphism $f: \mathbb{T}^{n} \rightarrow \mathbb{T}^{n}$ is homotopic to a linear Anosov automorphism $A: \mathbb{T}^{n} \rightarrow \mathbb{T}^{n}$ we say that $f$ is a Derived from Anosov (DA) diffeomorphism. In this thesis we will always work with the class of absolutely partially hyperbolic diffeomorphisms, and for simplicity we will omit the term "absolutely".

Pesin showed in [62] that for a compact Riemannian manifold $M$, given a Kolmogorov diffeomorphism $f: M \rightarrow M$, if $f$ preserves a non uniformly hyperbolic smooth measure $\mu$ then $(f, \mu)$ is Bernoulli. That is, the presence of some type of hyperbolic behavior indeed provides the equivalence of Kolmogorov and Bernoulli 
property. Pesin's result was obtained by making a non-trivial and far reaching generalization of Ornstein-Weiss's ideas. The results of Ornstein-Weiss and the results of D. Rudolph on extension of Bernoulli shifts ( [54], [53]) have been used by several authors (for example [13], [35], [49], [50], [17]) to obtain Bernoulli property for different types of dynamical systems using the Kolmogorov property. However, the methods of Ornstein-Weiss do not apply when we have a center direction with non-trivial behavior as for diffeomorphisms in $\mathcal{P} \mathcal{H}_{m}^{r}\left(\mathbb{T}^{3}\right), r>1$.

In a recent paper A. Avila and M. Viana [2] studied the occurrence of Bernoulli property for $C^{1}$ perturbations of ergodic linear automorphisms of $\mathbb{T}^{4}$ with two complex eigenvalues of norm 1. They prove that ergodic linear automorphisms of $\mathbb{T}^{4}$ are stably Bernoulli, that is, every $C^{\infty}$ diffeomorphism $C^{1}$ close to the linear one is also Bernoulli. The technics developed by Avila-Viana to prove Bernoulli property in this case is the so called invariance principle. In this thesis, we study the Bernoulli property for partially hyperbolic diffeomorphisms (not necessarily close to a linear automorphism) on $\mathbb{T}^{3}$.

\subsection{Equivalence of Kolmogorov and Bernoulli prop- erty}

For a long time, mathematicians studied the difference or equivalence between Kolmogorov and Bernoulli properties in general settings. It was also D. Ornstein [41] who constructed the first example of a Kolmogorov but not Bernoulli automorphism and it is not a natural automorphism (that is, it is constructed specifically for this purpose). Later, a much more natural example was given by S. Kalikow [28]. Although Kalikow's example is natural, it is not a smooth function, and it was A. Katok [30] who constructed the first $C^{\infty}$ example in a smooth manifold that is Kolmogorov but not Bernoulli.

Knowing that Kolmogorov and Bernoulli properties are not equivalent has implications on a very important and deep problem in dynamics, the smooth realization problem. The smooth realization problem deals with the problem of obtaining measure conjugacy of measure preserving systems with a smooth diffeomorphism in a smooth compact manifold. By a result of Y. Pesin [62], every smooth diffeomorphism of a surface with positive entropy is Bernoulli, showing that positive entropy is not 
a sufficient condition for smooth realization, since Kalikow's example has positive entropy but is not Bernoulli.

After these results, most of the Kolmogorov diffeomorphisms appearing in the literature that admit some kind of hyperbolic behavior was shown to be Bernoulli. As cited before, many natural and important dynamical systems admit some type of hyperbolic behavior but are not non-uniformly hyperbolic, for example the time-1 map of a geodesic flow in a negatively curved surface.

Since the results of Ornstein [14] and Pesin [62] used in a strong way the non uniformly hyperbolic structure of the given dynamics, those results do not extend to partially hyperbolic dynamics and thus we are left with the following question:

Question 1.1.1. Are Kolmogorov and Bernoulli properties equivalent for the class of absolutely partially hyperbolic diffeomorphisms of a compact Riemannian manifold M? Is the time-1 map of the geodesic flow on negatively curved surfaces stably Bernoulli?

Let $m$ be the Lebesgue measure (volume measure) on $\mathbb{T}^{3}$. The Main Theorem of this thesis is a theorem proved jointly with A. Tahzibi and R. Varão [46] where we used geometric measure theory, ergodic theory and foliation theory to prove that for a large class of partially hyperbolic diffeomorphisms on $\mathbb{T}^{3}$ the Bernoulli property occurs.

Theorem A. ([46]) Let $f \in \mathcal{P} \mathcal{H}_{m}^{2}\left(\mathbb{T}^{3}\right)$ be homotopic to a linear Anosov. If $f$ is Kolmogorov, then $f$ is Bernoulli.

For $C^{2}$ partially hyperbolic diffeomorphisms on $\mathbb{T}^{3}$, the Kolmogorov property is equivalent to a very well known property, the essential accessibility property (see section 2.3.1), so that Theorem A could be also stated substituting "Kolmogorov" by "essentially accessible". This answers a specific case of a question raised by K. Burns during the congress Recent Progress in Dynamics - 2006, held in the Clay Mathematics Institute (see [23, section 11]).

In view of Theorem $\mathrm{A}$ and the leaf-conjugacy result proved by A. Hammerlindl [19], the only remaining case to complete the equivalence of Kolmogorov and Bernoulli property for partially hyperbolic diffeomorphisms on $\mathbb{T}^{3}$ is the case of compact center leaves. 
Conjecture 1.1.2. Every $C^{\infty}$ volume preserving Kolmogorov diffeomorphism of $\mathbb{T}^{3}$ is Bernoulli.

A very special case in the literature is the case of the time-1 map of the geodesic flow on negatively curved surfaces. It was shown by D. Ornstein [41] that this system was Bernoulli and K. Burns, C. Pugh and A. Wilkinson [11] showed that it is stably Kolmogorov. The natural final question to complete the ergodic classification of this map is:

Question 1.1.3. Is the time-1 map of the geodesic flow on a negatively curved surface stably Bernoulli?

As pointed out in [11] this question is still open. A result that may help to obtain conclusions in this direction is the dichotomy proved by A. Avila, M. Viana and A. Wilkinson [3], where they prove that a perturbation of the time-1 map of the geodesic flow is itself a time-1 map of an Anosov flow (thus Bernoulli), or the center foliation is atomic, that is we can find $k \in \mathbb{N}$ and a full measure set intersecting each center leaf in exactly $k$ points.

Conjecture 1.1.4. The time one map of a geodesic flow on a surface with negative curvature is stably Bernoulli.

\subsection{Disintegration of volume measure and patholog- ical examples}

The results of Ornstein and Pesin use in an essential way that the unstable and stable manifold of $f$ are absolutely continuous. In the partial hyperbolic setting we still have absolute continuity of unstable and stable directions but we have no information on the center direction, that is, the disintegration of the volume measure along the center direction is not known. This fact makes necessary a deeper analysis of this disintegration before trying to show the equivalence between those properties. More specifically, we show that the proof of the equivalence can be divided in two cases: pieces of collapsed center manifolds have full or zero volume. In the first case we prove that the disintegration is atomic and we show that actually the disintegration is mono-atomic. 
Also joint with A. Tahzibi and R. Varão [45] we proved a result concerning atomic disintegration that is more general than the one used in the proof of Theorem B. We prove that the atomic disintegration in the case of any ergodic DA diffeomorphism is actually mono-atomic.

Theorem B. ( [45]) Let $f \in \mathcal{P} \mathcal{H}_{m}^{2}\left(\mathbb{T}^{3}\right)$ be an ergodic DA diffeomorphism on $\mathbb{T}^{3}$. If the volume measure $m$ has atomic disintegration on the center leaves, then the disintegration is mono-atomic.

This result is interesting in its own since it classifies the atomic disintegration in the DA case. Together with A. Tahzibi [44] we constructed an open class of partially hyperbolic diffeomorphisms satisfying the conditions of Theorem B, thus yielding an open set of diffeomorphisms with minimal and measurable foliation, giving the first example of a minimal foliation of $\mathbb{T}^{3}$ that is measurable. Also, this class of diffeomorphisms shows a stronger type of Fubbini's nightmare phenomenon, since all occurrences of this phenomenon in the literature had bounded leaves.

Theorem C. ([44]) There exists a $C^{1}$-open set $U \subset \mathcal{P} \mathcal{H}_{m}^{\infty}\left(\mathbb{T}^{3}\right)$ such that for any $f \in U, f$ is ergodic, $f$ is homotopic to a linear Anosov and for almost every point $x \in \mathbb{T}^{3}$ we have

$$
\lambda_{f}^{c}(x) \lambda_{A}^{c}<0
$$

where $A: \mathbb{T}^{3} \rightarrow \mathbb{T}^{3}$ is the linearization of $f$.

Theorem D. ([45]) Let $f \in \mathcal{P H}_{m}^{2}\left(\mathbb{T}^{3}\right)$ be homotopic to an Anosov linear automorphism $A: \mathbb{T}^{3} \rightarrow \mathbb{T}^{3}$. If

$$
\lambda_{f}^{c}(x) \lambda_{A}^{c}<0
$$

for Lebesgue almost every point $x \in \mathbb{T}^{3}$, then volume has atomic disintegration on $\mathcal{F}_{f}^{c}$, in fact the disintegration is mono-atomic.

In the construction of Theorem $\mathrm{C}$ we also construct diffeomorphisms with zero center Lyapunov exponents and non-compact center leaves (see chapter 3). This is the first example of this type. 
Theorem E. ([44]) There exist $C^{\infty}$ volume preserving partially hyperbolic diffeomorphism $f: \mathbb{T}^{3} \rightarrow \mathbb{T}^{3}$, homotopic to linear Anosov automorphism, with zero central Lyapunov exponent for Lebesgue almost every point of $\mathbb{T}^{3}$.

\subsection{Structure of the thesis}

In Chapter 2 we state some fundamental background concepts and results in partially hyperbolic dynamics, ergodic theory and measure disintegration theory. In Chapter 3 we prove Theorem E showing how to construct examples of partially hyperbolic diffeomorphisms with zero center Lyapunov exponent Lebesgue almost everywhere, but homotopic to a linear Anosov. In the same construction we prove Theorem $\mathrm{C}$, giving examples of diffeomorphisms where the local asymptotic behavior on the center direction is opposite to the global asymptotic behavior.

In Chapter 4 we study the disintegration of volume along center foliation of ergodic DA diffeomorphisms and we prove Theorems B and D. The results of this chapter together with the construction of the pathological example yields an open class of diffeomorphisms having minimal foliation with only one atom per leaf, being the first examples of this type. These examples exhibit a surprising contrast between metric (measurable) properties and topological properties of foliations, showing how the theory of partially hyperbolic dynamics can help us to understand abstract problems and relations between the metric and topological theory of foliations. In Chapter 5 we prove Theorem A, showing that Kolmogorov DA's on $\mathbb{T}^{3}$ are actually Bernoulli. 


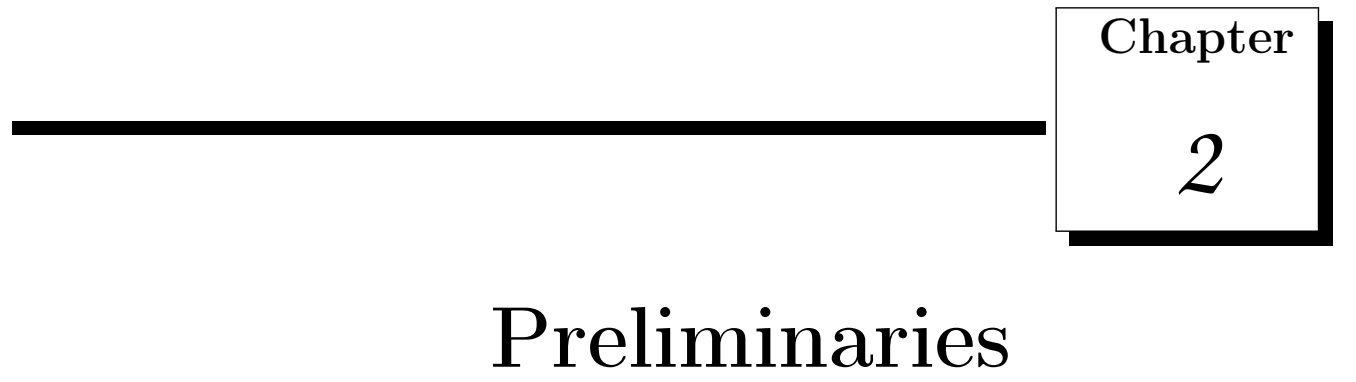

\subsection{Partially Hyperbolic Dynamics}

The ergodic theory of "beyond uniformly hyperbolic dynamics" is an extensive research area and has connections to many other topics. Partial hyperbolicity is a form of relaxing the uniform hyperbolicity condition with natural interesting examples (see [10], [24]). One of the amazing issues which comes up in the study of ergodic properties of partially hyperbolic dynamics is the existence of invariant foliations and their topological and metric properties. A complete comprehension of invariant foliations in partially hyperbolic dynamics is also an important tool for the classification of these dynamics and the manifolds which support them.

Let $M$ be a compact smooth manifold. A diffeomorphism $f: M \rightarrow M$ is partially hyperbolic if there exists a $D f$-invariant splitting of the tangent bundle

$$
T M=E^{s} \oplus E^{c} \oplus E^{u}
$$

such that $D f$ uniformly expands all vectors in $E^{u}$ and uniformly contracts all vectors in $E^{s}$, while vectors in $E^{c}$ are neither contracted as strongly as any nonzero vector in $E^{s}$ nor expanded as strongly as any nonzero vector in $E^{u} . f$ is called absolutely partially hyperbolic if the domination property between the three mentioned sub bundles is uniform on the whole manifold. More formally:

Definition 2.1.1. Given a smooth compact Riemannian manifold $M$. A diffeomor- 
phism $f: M \rightarrow M$ is called partially hyperbolic if the tangent bundle of the ambient manifold admits an invariant decomposition $T M=E^{s} \oplus E^{c} \oplus E^{u}$, such that all unit vectors $v^{\sigma} \in E_{x}^{\sigma}, \sigma \in\{s, c, u\}$ for all $x \in M$ satisfy:

$$
\left\|D_{x} f v^{s}\right\|<\left\|D_{x} f v^{c}\right\|<\left\|D_{x} f v^{u}\right\|
$$

and moreover $\left\|D f \mid E^{s}\right\|<1$ and $\left\|D f^{-1} \mid E^{u}\right\|<1$. We call $f$ absolutely partially hyperbolic, if it is partially hyperbolic and for any $x, y, z \in M$

$$
\left\|D_{x} f v^{s}\right\|<\left\|D_{y} f v^{c}\right\|<\left\|D_{z} f v^{u}\right\|
$$

where $v^{s}, v^{c}$ and $v^{u}$ belong respectively to $E_{x}^{s}, E_{y}^{c}$ and $E_{z}^{u}$.

Notation: Given $1 \leq r \leq \infty$ and a probability measure $\mu$ on $M$, we denote by $\mathcal{P} \mathcal{H}_{\mu}^{r}(M)$ the set of $C^{r}$ absolutely partially hyperbolic diffeomorphisms of $M$ preserving the probability measure $\mu$.

Along all the thesis, we will denote by $m$ the Lebesgue measure on $\mathbb{T}^{3}$. Thus $\mathcal{P H}_{m}^{r}(M)$ is the set of $C^{r}$ absolutely partially hyperbolic diffeomorphisms of $M$ which are volume preserving.

The set of absolutely partially hyperbolic diffeomorphism is $C^{1}$ open inside the set of all diffeomorphisms of $M$.

Definition 2.1.1. Given an orthogonal splitting of the tangent bundle of $M$

$$
E \oplus F=T M
$$

and a real constant $\beta>0$, for each $x \in M$ we define the cone centered in $E(x)$ with angle $\beta$ as

$C(x, E, \beta)=\left\{v \in T_{x} M:\left\|v_{F}\right\| \leq \beta\left\|v_{E}\right\|\right.$, where $\left.v=v_{E}+v_{F}, v_{E} \in E(x), v_{F} \in F(x).\right\}$

Absolutely partial hyperbolicity conditions can be expressed equivalently in terms of invariant cone families (see [64], pg.15).

Let $f: \mathbb{T}^{3} \rightarrow \mathbb{T}^{3}$ be a diffeomorphism. Then, $f$ is absolutely partially hyperbolic 
if, and only if, we can find a continuous orthogonal splitting of the tangent space

$$
T_{x} \mathbb{T}^{3}=E^{s}(x) \oplus E^{c}(x) \oplus E^{u}(x)
$$

a real number $0<\beta<1$ and constants

$$
0<\mu_{1}<\lambda_{2} \leq \mu_{2}<\lambda_{3}, \quad \mu_{1}<1, \quad \lambda_{3}>1
$$

for which the families of cones

$$
\begin{gathered}
C^{s}(x, \beta):=C\left(x, E^{s}(x), \beta\right), C^{u}(x, \beta):=C\left(x, E^{u}(x), \beta\right) \\
C^{c s}(x, \beta):=C\left(x, E^{c s}(x), \beta\right), C^{c u}(x, \beta):=C\left(x, E^{c u}(x), \beta\right)
\end{gathered}
$$

where $E^{c s}(x):=E^{c}(x) \oplus E^{s}(x), E^{c u}(x):=E^{c}(x) \oplus E^{u}(x)$, satisfy

$$
\begin{gathered}
D f^{-1}(x)\left(\mathcal{C}^{\tau}(x, \beta)\right) \subset \mathcal{C}^{\tau}\left(f^{-1}(x), \beta\right), \tau=s, c s \\
D f(x)\left(\mathcal{C}^{\Psi}(x, \beta)\right) \subset \mathcal{C}^{\Psi}(f(x), \beta), \Psi=u, c u
\end{gathered}
$$

and

$$
\begin{gathered}
\left\|D f^{-1}(x) v\right\|>\mu_{1}^{-1}\|v\|, v \in C^{s}(x, \beta) \\
\left\|D f^{-1}(x) v\right\|>\mu_{2}^{-1}\|v\|, v \in C^{c s}(x, \beta) \\
\|D f(x) v\|>\lambda_{3}\|v\|, v \in C^{u}(x, \beta) \\
\|D f(x) v\|>\lambda_{2}\|v\|, v \in C^{c u}(x, \beta) .
\end{gathered}
$$

We will use the characterization of absolutely partially hyperbolic diffeomorphisms by cone families in Chapter 3 .

Remark 2.1.2. In this thesis we always deal with absolutely partially hyperbolic diffeomorphisms, so we will omit the word "absolutely" and will refer to them only as partially hyperbolic diffeomorphisms.

It is well known that for partially hyperbolic diffeomorphisms, there are foliations $\mathcal{F}^{\tau}, \tau=s, u$, tangent to the sub-bundles $E^{\tau}, \tau=s, u$, called stable and unstable foliation respectively (for more details see for example [64]). On the other hand, the integrability of the central sub-bundle $E^{c}$ is a subtle issue and is not the case in the 
general partially hyperbolic setting (see [26]). However, by a result of M. Brin, D. Burago, S. Ivanov [7], all (absolutely) partially hyperbolic diffeomorphisms on $\mathbb{T}^{3}$ admit a central foliation tangent to $E^{c}$.

Let $f: \mathbb{T}^{3} \rightarrow \mathbb{T}^{3}$ be a partially hyperbolic diffeomorphism. Consider $f_{*}: \mathbb{Z}^{3} \rightarrow \mathbb{Z}^{3}$ the action of $f$ on the fundamental group of $\mathbb{T}^{3} \cdot f_{*}$ can be extended to $\mathbb{R}^{3}$ and the extension is the lift of a unique linear automorphism $A: \mathbb{T}^{3} \rightarrow \mathbb{T}^{3}$.

Definition 2.1.3. Given $f: \mathbb{T}^{3} \rightarrow \mathbb{T}^{3}$ a partially hyperbolic diffeomorphism. The unique linear automorphism $A: \mathbb{T}^{3} \rightarrow \mathbb{T}^{3}$ with lift $f_{*}: \mathbb{R}^{3} \rightarrow \mathbb{R}^{3}$ as constructed in the previous paragraph, is called the linearization of $f$.

It can be proved that the linearization $A$ of a partially hyperbolic diffeomorphism $f$, is a partially hyperbolic automorphism of torus ( [7]). It is not difficult to see that $f$ and $A$ behave similarly in large scale (see [20], corollary 2.2). More precisely, for each $k \in \mathbb{Z}$ and $C>1$ there is an $M>0$ such that for all $x, y \in \mathbb{R}^{3}$,

$$
\|x-y\|>M \Rightarrow \frac{1}{C}<\frac{\left\|\tilde{f}^{k}(x)-\tilde{f}^{k}(y)\right\|}{\left\|A^{k}(x)-A^{k}(y)\right\|}<C .
$$

where $\tilde{f}: \mathbb{R}^{3} \rightarrow \mathbb{R}^{3}$ is the lift of $f$ to $\mathbb{R}^{3}$. The examples in the open set $U$ of Theorem $\mathrm{C}$ show that in infinitesimal scales opposite behaviors can occur.

A. Hammerlindl proves that any absolutely partially hyperbolic diffeomorphism $f$ on $\mathbb{T}^{3}$ is leaf conjugated to its linearization (for higher dimensions see [19]). This means that there exist an homeomorphism $H: \mathbb{T}^{3} \rightarrow \mathbb{T}^{3}$ such that $H$ sends the central leaves of $f$ to central leaves of $f_{*}$ and conjugates the dynamics of the leaf spaces. In particular the central leaves of $f$ are all homeomorphic.

It is easy to see that a linear partially hyperbolic diffeomorphism of $\mathbb{T}^{3}$ is either Anosov or all of the leaves of $\mathcal{F}^{c}$ are compact, i.e, homeomorphic to $\mathbb{S}^{1}$. In the latter case the center eigenvalue is equal to one. In Theorem $\mathrm{C}$ we give an example of partially hyperbolic diffeomorphisms with zero central Lyapunov exponent almost everywhere and non compact center leaves.

Definition 2.1.2. We say that $f: \mathbb{T}^{3} \rightarrow \mathbb{T}^{3}$ is derived from Anosov or just a DA diffeomorphism if it is partially hyperbolic and its linearization is a hyperbolic automorphism (no eigenvalue of norm one). 


\subsubsection{Franks-Manning semi-conjugacy}

If $f: \mathbb{T}^{3} \rightarrow \mathbb{T}^{3}$ is a DA diffeomorphism, then by results of J. Franks [16] and A. Manning [34] there is a semi-conjugacy $h: \mathbb{T}^{3} \rightarrow \mathbb{T}^{3}$, which we will call throughout the thesis the Franks-Manning semi-conjugacy, between $f$ and its linearization $A$, that is,

$$
h \circ f=A \circ h .
$$

Moreover, this semi-conjugacy has the property that there exists a constant $K \in \mathbb{R}$ such that if $\tilde{h}: \mathbb{R}^{3} \rightarrow \mathbb{R}^{3}$ denotes the lift of $h$ to $\mathbb{R}^{3}$ we have $\|\tilde{h}(x)-x\| \leq K$ for all $x \in \mathbb{R}^{3}$, and given two points $a, b \in \mathbb{R}^{3}$, there exists a constant $\Omega>0$ with

$$
\tilde{h}(a)=\tilde{h}(b) \Leftrightarrow\left\|\tilde{f}^{n}(a)-\tilde{f}^{n}(b)\right\|<\Omega, \forall n \in \mathbb{Z} .
$$

Using this characterization of the semi-conjugacy and the quasi-isometry property (see definition 4.2.1) on the center foliation proved by A. Hammerlindl [19], R. Ures [59] proved that $h$ takes center leaves of $f$ onto center leaves of $A$, that is,

$$
\mathcal{F}_{A}^{c}(h(x))=h\left(\mathcal{F}_{f}^{c}(x)\right)
$$

Also by Franks [16] and Manning [34], if $f$ is an Anosov diffeomorphism then $h$ is actually a conjugacy between $f$ and its linearization $A$.

Definition 2.1.4. An f-invariant measure $\mu$ is called a measure of maximum entropy for $f$, or an entropy maximizing measure, if the metric entropy of $f$ with respect to $\mu$ is equal to the topological entropy of $f$, that is,

$$
h_{\mu}(f)=h_{\text {top }}(f) \text {. }
$$

Remark 2.1.5. We remark that the name "entropy maximizing measure" is motivated by the variational principle, which states that the topological entropy is the supremum of metric entropies (see Chapter 4 of [31] for more details on entropy and on the variational principle).

Also in [59], Ures concluded that $f$ is intrinsically ergodic, that is, there is a unique entropy maximizing measure $\mu$ for $f$ and this $\mu$ is natural in the sense that 
it satisfies

$$
h_{*} \mu=m,
$$

where $h$ is the Franks-Manning conjugacy between $f$ and its linearization $A$. Furthermore, $(f, \mu)$ is measurably conjugated to $(A, m)$ so that $(f, \mu)$ is Bernoulli.

In this context, although $f$ is intrinsically ergodic it is not known yet if $f \in$ $\mathcal{P H} \mathcal{H}_{m}^{2}\left(\mathbb{T}^{3}\right)$, homotopic to linear Anosov, must be ergodic for the volume measure. In this direction, A. Hammerlindl and R. Ures proved in a recent paper that nonergodicity implies topological conjugacy with the linear Anosov automorphism.

Theorem 2.1.6 (Propositions 4.2, 4.4 and Theorems 1.1, 6.1 and 7.2 of [22]). Let $f: \mathbb{T}^{3} \rightarrow \mathbb{T}^{3}$ be a $C^{2}$ volume preserving partially hyperbolic diffeomorphism, homotopic to a hyperbolic automorphism A. Assume $f$ is not ergodic. Then,

- $E^{s} \oplus E^{u}$ integrates to a minimal foliation;

- $f$ is topologically conjugate to $A$ and the conjugacy carries strong leaves of $f$ to the correspondent strong leaves of $A$;

- the central Lyapunov exponent of $f$ is zero for almost every point.

\subsection{Measurable partitions and disintegration of mea-}

\section{sures}

Let $(M, \mu, \mathcal{B})$ be a probability space, where $M$ is a compact metric space, $\mu$ a probability measure and $\mathcal{B}$ the borelian $\sigma$-algebra. Given a partition $\mathcal{P}$ of $M$ by measurable sets, we associate the probability space $(\mathcal{P}, \widetilde{\mu}, \widetilde{\mathcal{B}})$ by the following way. Let $\pi: M \rightarrow \mathcal{P}$ be the canonical projection, that is, $\pi$ associates to a point $x$ of $M$ the partition element of $\mathcal{P}$ that contains it. Then we define $\widetilde{\mu}:=\pi_{*} \mu$ and $\widetilde{\mathcal{B}}:=\pi_{*} \mathcal{B}$.

Definition 2.2.1. Given a partition $\mathcal{P}$. A family $\left\{\mu_{P}\right\}_{P \in \mathcal{P}}$ is a system of conditional measures for $\mu$ (with respect to $\mathcal{P}$ ) if

i) given $\phi \in C^{0}(M)$, then $P \mapsto \int \phi \mu_{P}$ is measurable;

ii) $\mu_{P}(P)=1 \widetilde{\mu}$-a.e.; 


$$
\text { iii) if } \phi \in C^{0}(M) \text {, then } \int_{M} \phi d \mu=\int_{\mathcal{P}}\left(\int_{P} \phi d \mu_{P}\right) d \widetilde{\mu} \text {. }
$$

When it is clear which partition we are referring to, we say that the family $\left\{\mu_{P}\right\}$ disintegrates the measure $\mu$.

Proposition 2.2.2. [15,51] Given a partition $\mathcal{P}$, if $\left\{\mu_{P}\right\}$ and $\left\{\nu_{P}\right\}$ are conditional measures that disintegrate $\mu$ on $\mathcal{P}$, then $\mu_{P}=\nu_{P} \widetilde{\mu}$-a.e.

Corollary 2.2.3. If $T: M \rightarrow M$ preserves a probability $\mu$ and the partition $\mathcal{P}$, then $T_{*} \mu_{P}=\mu_{T(P)}, \widetilde{\mu}-$ a.e.

Proof. It follows from the fact that $\left\{T_{*} \mu_{P}\right\}_{P \in \mathcal{P}}$ is also a disintegration of $\mu$.

Definition 2.2.4. We say that a partition $\mathcal{P}$ is measurable (or countably generated) with respect to $\mu$ if there exist a measurable family $\left\{A_{i}\right\}_{i \in \mathbb{N}}$ and a measurable set $F$ of full measure such that if $B \in \mathcal{P}$, then there exists a sequence $\left\{B_{i}\right\}$, where $B_{i} \in\left\{A_{i}, A_{i}^{c}\right\}$ such that $B \cap F=\bigcap_{i} B_{i} \cap F$.

Proposition 2.2.5. Let $(M, \mathcal{B}, \mu)$ a probability space where $M$ is a compact metric space and $\mathcal{B}$ is the Borel sigma-algebra. If $\mathcal{P}$ is a continuous foliation of $M$ by compact measurable sets, then $\mathcal{P}$ is a measurable partition.

Proof. This proposition has been proved in a preprint by Avila, Viana and Wilkinson. We put the argument here for the sake of completeness. Let $\left\{x_{i}\right\}$ be a countable dense subset of $M$. For each $x_{i}$ and $n \geq 1$, define $V\left(x_{i}, n\right)$ as the points $z \in M$ such that $\mathcal{P}_{z}$ intersects the closed ball of radius $\frac{1}{n}$ around $x_{i}$. It is easy to see that $V\left(x_{i}, n\right)$ is closed and hence measurable. Here we use the continuity of foliation. By definition $V\left(x_{i}, n\right)$ is a saturated subset, i.e it contains whole leaves of its points. For any two different leaves $\mathcal{P}_{x}$ and $\mathcal{P}_{y}$, take a large $n$ and $x_{i}$ such that $\mathcal{P}_{x}$ intersects the closed ball $B\left(x_{i}, \frac{1}{n}\right)$. By compactness of leaves, if $n$ is large enough $\left(\frac{2}{n}\right.$ is smaller than the distance between the leaves) then $B\left(x_{i}, \frac{1}{n}\right) \cap \mathcal{P}_{y}=\emptyset$.

Theorem 2.2.6 (Rokhlin's disintegration [51]). Let $\mathcal{P}$ be a measurable partition of a compact metric space $M$ and $\mu$ a borelian probability. Then there exists a disintegration by conditional measures for $\mu$. 
In general the partition by the leaves of a foliation may be non-measurable. It is for instance the case for the stable and unstable foliations of a linear Anosov diffeomorphism. Therefore, by disintegration of a measure along the leaves of a foliation we mean the disintegration on compact foliated boxes. In principle, the conditional measures depend on the foliated boxes, however, two different foliated boxes induce proportional conditional measures. See [3] for a discussion. We define absolute continuity of foliations as follows:

Definition 2.2.7. We say that a foliation $\mathcal{F}$ is absolutely continuous if for any foliated box, the disintegration of volume on the segment leaves have conditional measures equivalent to the Lebesgue measure on the leaf.

For the stable and unstable foliations of a partially hyperbolic diffeomorphis, it is well known (see [5], [64], [9], [47], [48]) that they are absolutely continuous and furthermore, the holonomy map between two transversals is absolutely continuous with Jacobian going to one as the transverses approach each other. We will make this statement more clear below.

Definition 2.2.8. Let $T: X \rightarrow Y$ be an invertible measurable transformation between two measure spaces $(X, \nu)$ and $(Y, \mu)$. We say that $G$ is absolutely continuous if $T_{*} \nu$ is absolutely continuous with respect to $\mu$. In this case, we define the Jacobian of $T$ at a point $x$ to be the Radon-Nikodym derivative

$$
\operatorname{Jac}(T)(x)=\frac{d \mu}{d T_{*} \nu}
$$

If $X$ is a metric space we have that for $\mu$-almost every $x \in X$

$$
\operatorname{Jac}(T)(x)=\lim _{r \rightarrow 0} \frac{\mu(T(B(x, r)))}{\nu(B(x, r))}
$$

Let $M$ be a compact Riemannian manifold and $f: M \rightarrow M$ be a $C^{1+\alpha}$ partially hyperbolic diffeomorphism. For fixed $x \in M, r>0$, consider the family of local manifolds (see section 4.3, and [5], or [64], for more details)

$$
\mathcal{L}(x)=\left\{\mathcal{W}_{\text {loc }}^{s}(w): w \in B(x, r)\right\}
$$


Choose two local disks, $D^{1}, D^{2}$ transverse to the family $\mathcal{L}(x)$.

Definition 2.2.9. The holonomy map generated by the local stable manifolds is the map $\pi^{s}=\pi^{s}(x): D^{1} \rightarrow D^{2}$ defined by setting

$$
\pi^{s}(y)=D^{2} \cap \mathcal{W}_{\mathrm{loc}}^{s}(w) \text { if } y=D^{1} \cap \mathcal{W}_{\mathrm{loc}}^{s}(w), w \in B(x, r)
$$

Given any submanifold $D$ of $M$, we denote by $m_{D}$ the Riemannian volume on $D$ induced by the restriction of the Riemannian metric to $D$.

Theorem 2.2.10 (see Theorem 8.6.1 of [5], Theorem 7.1 of [64]). Let $f$ be a $C^{1+\alpha}$ partially hyperbolic diffeomorphism of a compact smooth manifold $M$. Given $x \in M$ and two transverse disks $D^{1}$ and $D^{2}$ to the family $\mathcal{L}(x)$ of local stable manifolds $\mathcal{W}_{\text {loc }}^{s}(y), y \in B(x, r)$, the holonomy map $\pi^{s}: D^{1} \rightarrow D^{2}$ is absolutely continuous with respect to the Riemannian measures $m_{D^{1}}$ and $m_{D^{2}}$ of $D^{1}$ and $D^{2}$, and we can find a constant $K$ such that

$$
\left|\operatorname{Jac}\left(\pi^{s}\right)(y)-1\right| \leq K \Delta\left(D^{1}, D^{2}\right)
$$

where $\Delta\left(D^{1}, D^{2}\right)$ is the $C^{1}$ distance of the two transversals. In particular, $\mathcal{F}^{s}$ is absolutely continuous in the sense of definition 2.2.7. The same statement is true for the unstable foliation.

Definition 2.2.11. We say that a foliation $\mathcal{F}$ has atomic disintegration with respect to a measure $\mu$ if the conditional measures on any foliated box are a sum of Dirac measures.

Although the disintegration of a measure along a general foliation is defined in compact foliated boxes, it makes sense to say that the foliation $\mathcal{F}$ has a quantity $k_{0} \in \mathbb{N}$ of atoms per leaf. The meaning of "per leaf" should always be understood as a generic leaf, i.e., almost every leaf. That means that there is a set $A$ of $\mu$-full measure which intersects a generic leaf on exactly $k_{0}$ points. Let's see that this implies atomic disintegration. Definition 2.2.1 shows that it only make sense to talk about conditional measures from the generic point of view, hence when restricted to a foliated box $\mathfrak{B}$, the set $A \cap \mathfrak{B}$ has $\mu$-full measure on $\mathfrak{B}$, therefore the support of 
the conditional measure disintegrated on $\mathfrak{B}$ must be contained on the set $A$. This implies atomic disintegration.

It is worth remarking that the weight of an atom for a conditional measure naturally depends on the foliated box, but the fact that a point $x$ is an atom is independent of the foliated box where we disintegrate a measure and by Corollary 2.2.3 the set of atoms is invariant under the dynamics. For a more detailed discussion about dependence of disintegration on the foliated box see Lemma 3.2 of [3].

\subsection{Ergodic Hierarchy}

In this section we will briefly state some basic facts of ergodic theory and we refer to Chapter 7 of the book [29] for a more detailed discussion. Though most of the concepts involved here are fairly well understood and can be found in any standard ergodic theory book, we opted to put them here to make more clear the context we are working with and the problems involved in this context. Since in smooth ergodic theory the Kolmogorov property is not so explored in its essence, we will focus more on this property providing some standard results of ergodic theory relating Kolmogorov property with entropy and also the results relating the Kolmogorov property with the presence of hyperbolic structures.

Ergodic hierarchy is a way of classifying the different degrees of mixing that a system can have. Usually five hierarchy classes are studied, which we define below.

Definition 2.3.1. - Given $\left\{\mathcal{P}_{\omega}\right\}_{\omega \in \Omega}$ a family of $\sigma$-algebras of the same space $X$, the join of the $\sigma$-algebras $\mathcal{P}_{\omega}$, denoted by

$$
\bigvee_{\omega \in \Omega} \mathcal{P}_{\omega}
$$

is the $\sigma$-algebra generated by the family of sets

$$
\left\{\bigcap_{i=1}^{n} P_{\omega_{i}}: P_{\omega_{i}} \in \mathcal{P}_{\omega_{i}}, w_{i} \in \Omega\right\}
$$

- Given two finite partitions $\alpha, \beta$ of the same space $X$, the join of $\alpha$ and $\beta$, denoted by $\alpha \vee \beta$, is the partition into sets $A \cap B, A \in \alpha, B \in \beta$. 
- Given an infinite family of finite partitions $\left\{\alpha_{\omega}\right\}_{\omega \in \Omega}$ of partitions of the same space $X$, the join of the partitions $\alpha_{\omega}$, denoted by

$$
\bigvee_{\omega \in \Omega} \alpha_{\omega}
$$

is the smallest $\sigma$-algebra containing all the elements of the partitions $\alpha_{\omega}, \omega \in$ $\Omega$.

Definition 2.3.2. Let $(X, \mu)$ be a probability measurable space with $\sigma$-algebra $\mathcal{A}$ and $T: X \rightarrow X$ a $\mu$-preserving measurable transformation.

1. $T$ is ergodic with respect to $\mu$ if: given any $A \in \mathcal{A}$ with $T(A)=A$ then $\mu(A) \in\{0,1\}$.

2. $T$ is weakly mixing with respect to $\mu$ if given $A, B \in \mathcal{A}$

$$
\lim _{n \rightarrow+\infty} \frac{1}{n} \sum_{i=0}^{n-1}\left|\mu\left(A \cap T^{-i}(B)\right)-\mu(A) \mu(B)\right|=0
$$

3. $T$ is mixing with respect to $\mu$ if for any $A, B \in \mathcal{A}$ one has

$$
\lim _{n \rightarrow \infty} \mu\left(T^{-n}(A) \cap B\right)=\mu(A) \mu(B) .
$$

4. $T$ is Kolmogorov with respect to $\mu$, or simply a K-automorphism, if there is a sub- $\sigma$-algebra $\mathcal{K} \subset \mathcal{A}$ such that

- $T^{-1} \mathcal{K} \subset \mathcal{K}$

- $\bigvee_{i=0}^{\infty} T^{i} \mathcal{K}=\mathcal{A}$ and

- $A \in \bigcap_{i=0}^{\infty} T^{-i} \mathcal{K} \Rightarrow \mu(A) \in\{0,1\}$.

5. $T$ is Bernoulli with respect to $\mu$, or simply a Bernoulli automorphism, if it is isomorphic to a Bernoulli shift. 
Theorem 2.3.3 (see for example, Chapter 7 of [29]).

Bernoulli $\subsetneq$ Kolmogorov $\subsetneq$ Mixing $\subsetneq$ Weak Mixing $\subsetneq$ Ergodic.

The diagram in Theorem 2.3.3 is intended to indicate that every Bernoulli automorphism is a Kolmogorov automorphism, every Kolmogorov automorphism is Mixing, and so on. As one can note, the relations on Theorem 2.3.3 are strict. In Chapter 7 of [29] the reader may see a detailed construction of examples that belong to a hierarchy class but not to the inferior one. We describe the basic examples here following [29] and omitting the proofs.

Example 2.3.4 (Ergodic but not weakly mixing). Given any irrational number $\alpha>0$ the rotation $R_{\alpha}: S^{1} \rightarrow S^{1}$ on the circle is ergodic but not weak mixing.

Example 2.3.5 (Weakly mixing but not Mixing). This example is constructed by a simple cutting and stacking process (for details on cutting and stacking see Chapter 2 of [29]). Let $T_{1}$ be a tower having one rung; the interval [0,1). Having constructed $T_{n}$, construct $T_{n+1}$ as follows. Break $T_{n}$ into $n+1$ equal width columns $C_{1}, \ldots, C_{n+1}$. Stack the columns vertically, starting with $C_{1}, \ldots, C_{n+1}$. Put a single spacer between $C_{n}$ and $C_{n+1}$. Normalize to a probability measure and denote the system this cutting and stacking construction converges to by $(X, T)$. This $(X, T)$ is weakly mixing but not mixing.

Example 2.3.6 (Mixing but not Kolmogorov). The example we show here is due to T. Adams [1]. Define a system by cutting and stacking as follows. Let $T_{1}=[0,1)$. We view this as a tower having a single rung. To get $T_{2}$ from $T_{1}$, cut $T_{1}$ into two sub columns and stack them, putting a spacer in between. To get $T_{3}$ from $T_{2}$, cut $T_{2}$ into three sub columns and stack them, putting one spacer in between the first two sub columns and two spacers between the last two sub columns. Having constructed $T_{n-1}$, cut it into $n$ sub columns $C_{1}, \ldots, C_{n}$ and stack them, putting $i$ spacers between $C_{i}$ and $C_{i+1}$. This gives $T_{n}$; denote its height by $h_{n}$. Finally, normalize the measure; the resulting system $(X, \mathcal{A}, \mu, T)$ is called the staircase. The staircase is a mixing system with zero entropy, thus it is not Kolmogorov. 
The class of Kolmogorov systems was introduced by Kolmogorov in 1958 and until 1969 it was not known if every Kolmogorov system was a Bernoulli system. The first example of a Kolmogorov but not Bernoulli automorphism was presented by D. Ornstein [41]. Ornstein's example is not easy to exhibit and not natural. The example we show below is known as $\left(T, T^{-1}\right)$ process. This natural example arose in 1971, and mathematicians at that time knew that it was a Kolmogorov automorphism, but the question of whether it was Bernoulli or not remained open until 1982 when S. Kalikow proved in [28] that this automorphism is not a Bernoulli automorphism. Though the definition of this process is very simple the proof that it is a Kolmogorov non-Bernoullian system is far from being easy.

Example 2.3.7 (Kolmogorov but not Bernoulli). The system we construct here is called the $\left(T, T^{-1}\right)$-process. The construction is simple and is described in several standard books of ergodic theory.

Let $\sigma: \Sigma \rightarrow \Sigma$ the shift map in $\Sigma=\{0,1\}^{\mathbb{Z}}$. Let $\mu$ be the standard Bernoulli measure given by the probability vector $(1 / 2 ; 1 / 2)$. Define $f: \Sigma \times \Sigma \rightarrow \Sigma \times \Sigma$ by

$$
\begin{gathered}
f\left(\left\{x_{n}\right\}_{n},\left\{y_{n}\right\}_{n}\right)=\left(\sigma\left(\left\{x_{n}\right\}_{n}\right), \sigma\left(\left\{y_{n}\right\}_{n}\right)\right), \text { if } x_{0}=0 \\
f\left(\left\{x_{n}\right\}_{n},\left\{y_{n}\right\}_{n}\right)=\left(\sigma\left(\left\{x_{n}\right\}_{n}\right), \sigma^{-1}\left(\left\{y_{n}\right\}_{n}\right)\right), \text { if } x_{0}=1 .
\end{gathered}
$$

This process $(f, \mu \times \mu)$ is often called a random walk on a random scenery.

\subsubsection{Kolmogorov property and partial hyperbolicity}

Though Kolmogorov property is not as intuitive as the other ergodic properties, one can see the Kolmogorov property using entropy. The following Theorem of $\mathrm{V}$. Rokhlin and Y. Sinai shows that Kolmogorov property is a sufficient condition to have uncertainty of a system in the sense that every finite partition generates a positive information.

Theorem 2.3.8 (Rokhlin-Sinai, [52]). Let $(X, \mu)$ be a Lebesgue space (i.e, isomorphic to $([0,1]$, Leb) ). An automorphism $f:(X, \mu) \rightarrow(X, \mu)$ is Kolmogorov if, and only if, it has completely positive entropy, that is, given any non-trivial finite 
partition $\mathfrak{A}$ of $X$ we have

$$
h_{\mu}(f, \mathfrak{A})>0
$$

Definition 2.3.9. Let $(X, \mu)$ be a Lebesgue space and $T:(X, \mu) \rightarrow(X, \mu)$ a measure preserving automorphism. The family $\pi$ defined by

$$
\pi=\left\{A \subset X: h_{\mu}(T, \alpha)=0, \alpha:=\{A, X-A\}\right\}
$$

is a $\sigma$-algebra called the Pinsker $\sigma$-algebra of $T$.

An immediate consequence of Theorem 2.3.8 is:

Theorem 2.3.10. $T:(X, \mu) \rightarrow(X, \mu)$ is a Kolmogorov automorphism if, and only if, the Pinsker partition $\pi$ of $T$ is trivial, i.e, every $A \in \pi$ has $\mu(A) \in\{0,1\}$.

The Kolmogorov property got a new status in smooth ergodic theory after the works of Pesin [63] and Ledrappier-Young [33] where they related the Pinsker partition of a partially hyperbolic diffeomorphism with the invariant foliations of this diffeomorphism.

Definition 2.3.11. Given two $\sigma$-algebras $\mathcal{C}, \mathcal{D}$ in a measurable space $(X, \mu)$, we

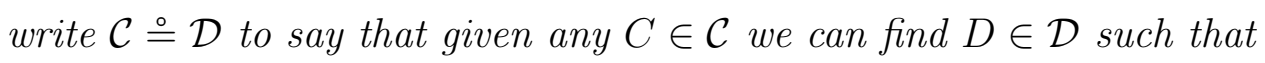

$$
\mu(C \Delta D)=0
$$

In [9], M. Brin and Y. Pesin showed that for a $C^{1+\alpha}$ partially hyperbolic diffeomorphism $f: M \rightarrow M$ ( $M$ a compact Riemannian manifold), every element of the pinsker $\sigma$-algebra is essentially bi-saturated. In [33] F. Ledrappier and L. Young proved the following:

Theorem 2.3.12 (Ledrappier-Young [33]). Let $M$ be a compact Riemannian manifold and $f: M \rightarrow M$ a $C^{2}$ diffeomorphism preserving a Borel probability measure $\mu$. Then

$$
\text { Pinsker } \sigma-\text { algebra of } f \stackrel{\circ}{=} \mathcal{B}^{u} \stackrel{\circ}{=} \mathcal{B}^{s} \text {, }
$$

where $\mathcal{B}^{\tau}$ denotes the $\sigma$-algebra of $\mathcal{F}^{\tau}$ saturated sets, $\tau=s, u$. 
We remark that Theorem 2.3.12 was proved by Y. Pesin in [62] for $C^{2}$ partially hyperbolic diffeomorphisms preserving a smooth probability measure $\mu$.

The characterization of the pinsker partition obtained by Brin-Pesin and LedrappierYoung is very useful to find ergodicity using Hopf-like arguments, as done for example in [12] and [25]. This characterization is used together with the definition of accessibility or essential accessibility which we define below.

Definition 2.3.13. Given a partially hyperbolic diffeomorphism $f: M \rightarrow M$ and $a$ point $x \in M$. The accessibility class of $x, A C(x)$ is the set of all points $y \in M$ such that we can find a finite number of points

$$
x_{0}=x, x_{1}, x_{2}, \ldots, x_{k-1}, x_{k}=y
$$

such that for $i=0, \ldots, k-1$,

$$
x_{i+1} \in \mathcal{F}^{u}\left(x_{i}\right) \cup \mathcal{F}^{s}\left(x_{i}\right) .
$$

Definition 2.3.14. We say that a partially hyperbolic diffeomorphism $f \in \mathcal{P F}^{r}(M)_{\mu}$ is accessible if for any $x \in M$ we have

$$
A C(x)=M
$$

We say that $f$ is essentially accessible if any measurable union of accessibility classes have either measure zero or one.

Definition 2.3.15. Let $f: M \rightarrow M$ be a partially hyperbolic diffeomorphism. We say that $f$ is center bunched if there exists a certain Riemannian metric and real constants $\nu, \gamma, \hat{\gamma}, \hat{\nu}$ with

$$
\begin{gathered}
\nu, \hat{\nu}<1,, \nu<\gamma<\hat{\gamma}^{-1}<\hat{\nu}^{-1} \\
\max \{\nu, \hat{\nu}\}<\gamma \hat{\gamma}
\end{gathered}
$$


and such that for any $x \in M$ and any unit vector $v \in T_{x} M$

$$
\begin{array}{rr}
\|D f(x) v\|<\nu, & \text { if } v \in E^{s}(x) \\
\gamma<\|D f(x) v\|<\hat{\gamma}^{-1}, & \text { if } v \in E^{c}(x) \\
\hat{\nu}^{-1}<\|D f(x) v\|, & \text { if } v \in E^{u}(x) .
\end{array}
$$

Remark 2.3.16. Here we are always working with absolute partial hyperbolicity. In the non-absolute case the definition of center bunched is similar, but involving functions instead of constants (see [12]).

The following Theorem by K. Burns and A. Wilkinson shows that under the hypothesis of being center bunched, essential accessibility of a $C^{2}$ partially hyperbolic diffeomorphism guarantees that the system is Kolmogorov.

Theorem 2.3.17 (Burns-Wilkinson [12]). Let $f$ be $C^{2}$, volume-preserving, partially hyperbolic and center bunched. If $f$ is essentially accessible, then $f$ is ergodic, and in fact has the Kolmogorov property.

For the one-dimensional center case, a stronger type of center bunching hypothesis is trivially satisfied and the $C^{2}$ condition can be weakened to $C^{1+\alpha}$ (see [12] for details).

Corollary 2.3.18 (Corollary 0.4 of [12]). Let $f$ be a $C^{1+\alpha}$, volume preserving and partially hyperbolic with $\operatorname{dim}\left(E^{c}\right)=1$. If $f$ is essentially accessible, then $f$ is ergodic, and in fact has the Kolmogorov property.

Corollary 2.3.19. Let $f: \mathbb{T}^{3} \rightarrow \mathbb{T}^{3}$ be a volume preserving $C^{1+\alpha}$ partially hyperbolic diffeomorphism. Thus $f$ is Kolmogorov if, and only if, it is essentially accessible.

Proof. By [62] we know that Kolmogorov property implies that every $u$-saturated set has measure zero or one. Since union of accessibility classes are in particular $u$-saturated they must have zero or full measure, thus it is essentially accessible. The other way is a consequence of Theorem 2.3.17 since $f$ is trivially center bunched. 
The following Theorem by F. Hertz, M. A. Hertz and R. Ures shows that for the one dimensional center case, the accessibility hypothesis (consequently the Kolmogorov hypothesis) is $C^{r}$ open and dense in the set of volume preserving diffeomorphisms, for any $1 \leq r \leq \infty$.

Theorem 2.3.20 (Hertz-Hertz-Ures [25]). For all $1 \leq r \leq \infty$, accessibility is open and dense in the set of $C^{r}$ partially hyperbolic diffeomorphisms on a compact Riemannian manifold $M$, preserving a smooth probability measure $m$, with one dimensional center distribution.

As we have seen above, the Kolmogorov and Bernoulli property are not equivalent in general. However, D. Ornstein and B. Weiss [14] proved that the geodesic flow in a negatively curved surface is Bernoulli and the technique they used was generalized in [43], and by Y. Pesin in [62].

Theorem 2.3.21 ([14]). Let $M$ be a Riemannian surface with negative curvature and $X$ its unit tangent bundle. Then, the geodesic flow $\phi: X \rightarrow X$ is Bernoulli, that is, the time one map $\phi_{1}$ is Bernoulli.

Theorem 2.3.22 ( [62], Theorem 9.1 of [5]). Let $f$ be a $C^{1+\alpha}$ diffeomorphism of a smooth compact Riemannian manifold $M$ preserving an absolutely continuous hyperbolic measure $\nu$ (that is, for $\nu$-almost every point $x$, the Lyapunov exponents at $x$ are non zero). Assume that $f$ is a Kolmogorov automorphism. Then $f$ is a Bernoulli automorphism.

The argument used by Ornstein-Weiss, is essentially the only method (that is, it is the basis) to obtain equivalence of Kolmogorov and Bernoulli properties in the smooth ergodic theory context. The results of Ornstein-Weiss and the results of D. Rudolph on extension of Bernoulli shifts ( [54], [53]) has been used by several authors (for example [13], [35], [49], [50], [17]) to obtain Bernoulli property for different types of dynamical systems using the Kolmogorov property. However, the method of Ornstein and Weiss does not apply when we have a center direction with nontrivial behavior as for diffeomorphisms in $\mathcal{P} \mathcal{H}_{m}^{r}\left(\mathbb{T}^{3}\right), r>1$. The advantage of working with DA diffeomorphisms is that we can compare the behavior of the linear diffeomorphism with the behavior of the diffeomorphism itself by using FranksManning semi-conjugacy. We use this semi-conjugacy to obtain informations on the 
center behavior of the diffeomorphism and then use an adaptation of Pesin's proof to conclude Bernoulli property. 


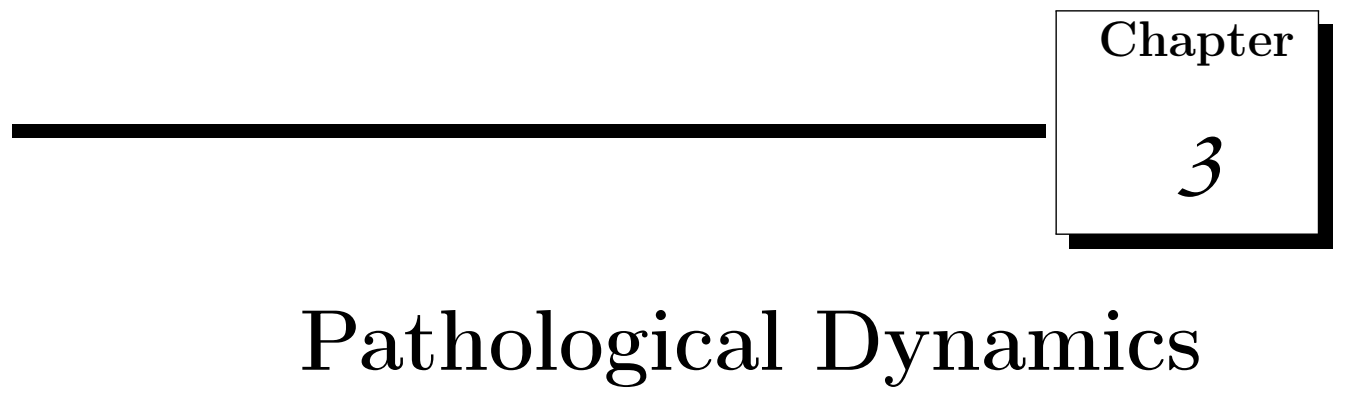

In this chapter we introduce some new "pathological" examples of partially hyperbolic diffeomorphisms. We study the relationship between central Lyapunov exponents and topology of leaves of central foliation of a partially hyperbolic diffeomorphism and its linearization. More precisely, we find an open set of partially hyperbolic diffeomorphisms $f: \mathbb{T}^{3} \rightarrow \mathbb{T}^{3}$ homotopic to linear Anosov diffeomorphisms $A$ such that the central Lyapunov exponent of $f$ is positive almost everywhere while the central bundle of $A$ is contracting. This opposite behavior in the asymptotic growth (manifested by the sign of Lyapunov exponent) which is a local issue contrasts with the compatible behavior in the large scale between $f$ and $A$ (see Section 2.1).

We also obtain examples of partially hyperbolic diffeomorphisms homotopic to Anosov with non compact central leaves and zero central Lyapunov exponent almost everywhere. This is also a contrast between the global topology of central leaves of non linear and linearization of a partially hyperbolic diffeomorphism. We recall the statements of the theorems proved in this chapter.

Theorem C. ([44]) There exists a $C^{1}$-open set $U \subset \mathcal{P} \mathcal{H}_{m}^{\infty}\left(\mathbb{T}^{3}\right)$ such that for any $f \in U, f$ is ergodic, $f$ is homotopic to a linear Anosov and for almost every point $x \in \mathbb{T}^{3}$ we have

$$
\lambda_{f}^{c}(x) \lambda_{A}^{c}<0
$$

where $A: \mathbb{T}^{3} \rightarrow \mathbb{T}^{3}$ is the linearization of $f$. 
Theorem E. ([44]) There exist $C^{\infty}$ volume preserving partially hyperbolic diffeomorphism $f: \mathbb{T}^{3} \rightarrow \mathbb{T}^{3}$, homotopic to linear Anosov automorphism, with zero central Lyapunov exponent for Lebesgue almost every point of $\mathbb{T}^{3}$.

Another interesting aspect of the diffeomorphisms inside $U$ is related to absolute continuity of central foliation. We do not know whether the central foliation of diffeomorphisms with zero central exponent in Theorem $\mathrm{E}$ is absolutely continuous or not. We believe that it is not the case and formulate the following question.

Question 3.0.23. Let $f: M \rightarrow M$ be a partially hyperbolic diffeomorphism of $M=$ $\mathbb{T}^{3}$ with absolutely continuous central foliation $\mathcal{F}^{c}$ and central Lyapunov exponent equal to zero at Lebesgue almost every point. Is it true that the leaves of $\mathcal{F}^{c}$ are compact?

We remark that A. Tahzibi and F. Micena [36] gave an affirmative answer to question 3.0.23 assuming $\mathcal{F}^{c}$ satisfies a uniformly bounded density condition which is a regularity condition stronger than leafwise absolute continuity.

The idea of the proof of Theorems $\mathrm{C}$ and $\mathrm{E}$ is to take a family of hyperbolic linear automorphisms $f_{k}: \mathbb{T}^{3} \rightarrow \mathbb{T}^{3}$ with eigenvalues $\lambda_{k}^{s}<\lambda_{k}^{c}<1<\lambda_{k}^{u}$ in such a way that

$$
\lambda_{k}^{s} \rightarrow 0, \lambda_{k}^{c} \rightarrow 1, \lambda_{k}^{u} \rightarrow \infty
$$

as $k \rightarrow \infty$ and moreover the corresponding unitary eigenvectors converge to a fixed orthonormal basis. Then we apply Baraviera-Bonatti [4] method of local perturbations and by the choice of the Anosov automorphisms, we are able to show that this local perturbation yields a new partially hyperbolic diffeomorphism with positive central Lyapunov exponent on average. By a continuity argument we find some homotopic to Anosov and partially hyperbolic diffeomorphism with vanishing integral of central Lyapunov exponent.

Finally, by Theorem 2.1.6 the diffeomorphisms obtained in Theorem C are ergodic and so almost every point have the same center Lyapunov exponent. For diffeomorphisms obtained in Theorem E, with vanishing average of central exponent, even without proving the ergodicity we obtain vanishing Lyapunov exponent almost everywhere as a consequence of Theorem 2.1.6. Later we prove that they are indeed ergodic. 


\subsection{Local Perturbation}

In this section we describe briefly a local perturbation process introduced by A. Baraviera, C. Bonatti [4]. For any partially hyperbolic diffeomorphism $f_{0}$ they construct a $C^{1}$-arc of diffeomorphisms $\left\{f_{r}\right\}$ for which the integral of central Lyapunov exponent of $f_{r}$ is strictly bigger than integral of the central exponent of $f_{0}$.

In [4], this perturbation procedure is made in a general case. Here we will use the perturbation argument just for the linear case.

Let $f: \mathbb{T}^{3} \rightarrow \mathbb{T}^{3}$ be a volume preserving, linear partially hyperbolic diffeomorphism. Denote by $\lambda^{s}<\lambda^{c}<\lambda^{u}$ the eigenvalues of $f$ and its unitary eigenvectors by $e_{s}, e_{c}, e_{u}$ respectively. Thus, the directions of $e_{s}, e_{c}, e_{u}$ are the directions of the subbundles $E^{s}, E^{c}, E^{u}$. Let $p$ be a non-fixed point. We take a $C^{1}$-local coordinate system on a neighborhood $V$ centered at $p$ such that $\left\{e_{s}, e_{c}, e_{u}\right\}$ are directed by $\frac{\partial}{\partial x}, \frac{\partial}{\partial y}, \frac{\partial}{\partial z}$. Moreover, the expression of volume form on $\mathbb{T}^{3}$ coincides with the Lebesgue measure on $\mathbb{R}^{3}$.

Let $B_{1}(0)$ be the unit ball of $\mathbb{R}^{3}$. Given any ball $B_{r}(p)$ inside $V$ we denote by $\varphi_{r}: B_{r}(p) \rightarrow B_{1}(0)$ the diffeomorphism which in local coordinates is a homothety of ratio $\frac{1}{r}$. More precisely, if $\pi: V \rightarrow \mathbb{R}^{3}$ is the mentioned coordinate system $\varphi_{r}(x):=$ $\frac{\pi(x)}{r}$.

Let $h: B_{1}(0) \rightarrow B_{1}(0), h \neq \mathrm{Id}$, a volume preserving diffeomorphism which preserves the $x$-direction, and equal to the identity in a neighborhood of the boundary of $B_{1}(0)$.

We define the diffeomorphism $h_{r}: \mathbb{T}^{3} \rightarrow \mathbb{T}^{3}$ by:

$$
h_{r}(w)= \begin{cases}w & , \text { if } w \notin B_{r}(p) \\ \varphi_{r}^{-1} \circ h \circ \varphi_{r}(w) & , \text { if } w \in B_{r}(p) .\end{cases}
$$

Finally, we define the arc of diffeomorphisms $\left\{f_{r}\right\}_{r \in[0,1]}$ by:

$$
f_{r}:=f \circ h_{r}
$$

Also, we take $h$ to satisfy

$$
\|h-\mathrm{Id}\|_{C^{1}}<1
$$


Since $h$ preserves the direction of $e_{s}$ we can write

$$
D h(p) e_{u}=h^{u}(p) e_{u}+h^{c}(p) e_{c}
$$

Lemma 3.1.1 ( [4]). Let $h$ be as above, then

$$
I(h):=\int_{B_{1}(0)} \log h^{u}(p) d m(p)<0 .
$$

Consider $n_{r}$ the least positive integer such that

$$
f^{n_{r}}\left(B_{r}\right) \cap B_{r} \neq \emptyset
$$

Denote by $\lambda_{r}^{u}(p)$ the unstable Lyapunov exponent of $f_{r}$ at $p$ and define:

$$
\sigma_{f_{r}}^{u}=\int \log J_{f_{r}}^{u}(p) d m(p), \quad \sigma_{f_{r}}^{c}=\int \log J_{f_{r}}^{c}(p) d m(p)
$$

where $J_{f_{r}}^{\tau}(p)$ denotes the Jacobian of $f_{r}$ on $E_{f_{r}}^{\tau}(p)$, that is, the modulus of the determinant of the restriction of $D f_{r}(p)$ to $E_{f_{r}}^{\tau}(p), \tau=s, c, u$.

Lemma 3.1.2 ( [4]). Let $\sigma_{f_{r}}^{u}$ and $\sigma_{f_{r}}^{c}$ be as above, then

$$
\log \lambda^{u}-\sigma_{f_{r}}^{u} \geq m\left(B_{r}\right)\left(-I(h)-C \alpha^{n_{r}}\right)
$$

where $\alpha=\lambda^{c} / \lambda^{u}$, and $C=\max _{x \in B_{r}} \frac{h_{r}^{c}}{h_{r}^{u}} \cdot \max _{x \in B_{r}}\left\|\operatorname{Proj}_{u}\left(e_{c}\right)\right\|$ with $\operatorname{Proj}_{u}\left(e_{c}\right)$ denoting the projection of $e_{c}$ over $E^{u}$ parallel to the new center bundle.

Observe that by (3.3) the perturbation $h_{r}$ preserves the center unstable bundle and the center-unstable jacobian of $h_{r}$ is equal to one. So the integral of the logarithm of the jacobian of $f_{r}$ in the center-unstable direction is the same of the one for $f$ (see [4], pg.1664). Thus, we have the following corollary.

Corollary 3.1.3. With the previous notations, the difference between $\sigma_{f_{r}}^{c}$ and $\log \lambda^{c}$ is bounded from below as follows:

$$
\sigma_{f_{r}}^{c}-\log \lambda^{c}=\log \lambda^{u}-\sigma_{f_{r}}^{u} \geq m\left(B_{r}(p)\right) \cdot\left(-I(h)-C \cdot\left(\frac{\lambda^{c}}{\lambda^{u}}\right)^{n_{r}}\right) .
$$




\subsection{Family of Linear Anosov Automorphisms}

In order to realize a perturbation that changes the sign of the central Lyapunov exponent, it is reasonable to take a diffeomorphim with central exponent close to 0 and big unstable exponent (so that we can borrow some hyperbolicity from the unstable direction). For each $k \in \mathbb{Z}$ define the linear automorphism $f_{k}: \mathbb{T}^{3} \rightarrow \mathbb{T}^{3}$ induced by the integer matrix:

$$
A_{k}=\left(\begin{array}{ccc}
0 & 0 & 1 \\
0 & 1 & -1 \\
-1 & -1 & k
\end{array}\right)
$$

The characteristic polynomial of $A_{k}$ is

$$
p_{k}(x)=x^{3}-(k+1) x^{2}+k x-1 \text {. }
$$

Lemma 3.2.1. For all $k \geq 5, A_{k}$ has real eigenvalues $0<\lambda_{k}^{s}<\lambda_{k}^{c}<1<\lambda_{k}^{u}$ and

$$
\lambda_{k}^{s} \rightarrow 0, \lambda_{k}^{c} \rightarrow 1, \lambda_{k}^{u} \rightarrow \infty
$$

as $k \rightarrow \infty$.

Proof. First of all note that:

- $p_{k}(1 / 2)=\frac{k}{4}-\frac{9}{8}>0, \forall k \geq 5$;

- $p_{k}(1)=p_{k}(k)=-1, \forall k$;

- $p_{k}(k+1)=k(k+1)-1 \geq 1, \forall k \geq 1$.

So, for all $k \geq 5, p_{k}$ has a root $\lambda_{k}^{u} \in(k, k+1)$ and a root $\lambda_{k}^{c} \in(1 / 2,1)$. Denoting by $\lambda_{k}^{s}$ the other root we have:

$$
0<\lambda_{k}^{s}=\frac{1}{\lambda_{k}^{c} \cdot \lambda_{k}^{u}}<\lambda_{k}^{c}<1<k<\lambda_{k}^{u} .
$$


Now, given any $0<\varepsilon<1$ we have

$$
p_{k}(1-\varepsilon)=k(1-\varepsilon) \varepsilon-\varepsilon(1-\varepsilon)^{2}-1
$$

which is trivially positive for large values of $k$. That is, $\lambda_{k}^{c} \rightarrow 1$ when $k \rightarrow \infty$. Also, since $k<\lambda_{k}^{u}$ and $\lambda_{k}^{s} \cdot \lambda_{k}^{c} \cdot \lambda_{k}^{u}=1$ we conclude that

$$
\lambda_{k}^{c} \rightarrow 1, \lambda_{k}^{u} \rightarrow \infty, \text { and } \lambda_{k}^{s} \rightarrow 0
$$

as $k \rightarrow \infty$.

Next, we evaluate the stable, central and unstable directions of $f_{k}$.

$$
A_{k} \cdot\left(\begin{array}{c}
a \\
b \\
c
\end{array}\right)=\left(\begin{array}{c}
\lambda a \\
\lambda b \\
\lambda c
\end{array}\right) \Rightarrow\left(\begin{array}{c}
a \\
b \\
c
\end{array}\right)=\left(\begin{array}{c}
a \\
\frac{\lambda a}{1-\lambda} \\
\lambda a
\end{array}\right)
$$

So the directions are $v_{k}^{\tau}:=\left(1, \lambda_{k}^{\tau} /\left(1-\lambda_{k}^{\tau}\right), \lambda_{k}^{\tau}\right)$ where $\tau=s, c, u$. Let $e_{\tau}^{k}:=\frac{v_{k}^{\tau}}{\left\|v_{k}^{\tau}\right\|}$, $\tau=s, c, u$. Then we have:

$$
e_{s}^{k} \rightarrow\left(\begin{array}{c}
1 \\
0 \\
0
\end{array}\right), e_{c}^{k} \rightarrow\left(\begin{array}{l}
0 \\
1 \\
0
\end{array}\right), e_{u}^{k} \rightarrow\left(\begin{array}{l}
0 \\
0 \\
1
\end{array}\right)
$$

as $k \rightarrow \infty$.

The following step is to apply local perturbations to each $f_{k}$. The aim of the next section is to define a family of functions $h_{k}$ that we will use to do the perturbation.

\subsection{Proof of Theorems C and E}

For a linear partially hyperbolic automorphim with eigenvalues $\lambda^{s}<\lambda^{c}<\lambda^{u}$, Corollary 3.1.3 implies that the following quantities are relevant to the amount of change of the central Lyapunov exponent after local perturbation method of Baraviera-Bonatti:

- $\alpha=\lambda^{c} / \lambda^{u}$ 
- $C=\max _{x \in B_{r}} \frac{h_{r}^{c}}{h_{r}^{u}} \cdot \max _{x \in B_{r}}\left\|\operatorname{Proj}_{u}\left(e_{c}\right)\right\|$;

- $m\left(B_{r}\right)$ and the return time $n_{r}$;

- $I(h)=\int_{B_{1}(0)} \log h^{u}(p) d m(p)$.

We consider the family $f_{k}$ constructed in the previous section. Recall that for each $k$ there exists an adapted inner product (which gives an adapted metric) where $e_{k}^{s}, e_{k}^{c}, e_{k}^{u}$ form an orthonormal set. As these eigenspaces are converging to the canonical basis the adapted metrics are close to the euclidean metric when $k$ is large enough. By euclidean metric we mean the usual inner product coming from euclidean inner product of $\mathbb{R}^{3}$. We will take a non-fixed point $p\left(f_{k}(p) \neq p\right)$ and $0<r<1$. We take a local coordinate $\pi_{k}: B_{r}^{k}(p) \rightarrow B_{r}(0) \subset \mathbb{R}^{3}$ such that the adapted inner product is the pullback by $D \pi_{k}$ of the euclidean inner product. Here $B_{r}^{k}$ is the ball of radius $r$ with respect to the adapted metric.

Take an arbitrary point

$$
p \in F:=P((0,2 / 3) \times(0,1) \times(5 / 6,1))
$$

where $P: \mathbb{R}^{3} \rightarrow \mathbb{T}^{3}$ is the canonical projection. Take fixed small $r$ such that $B_{r}^{k}(p) \in$ $F$ for large $k$. It is possible to find such $r>0$, because for large $k$ all the adapted metrics are close to the euclidean metric. It is easy to see that $f_{k}^{-1}(F) \cap F=\emptyset$ so $f_{k}(F) \cap F=\emptyset$ and $f_{k}\left(B_{r_{0}}^{k}(p)\right) \cap B_{r_{0}}^{k}(p)=\emptyset, \forall k \geq k_{0}$.

We take $h: B_{1}(0) \rightarrow B_{1}(0)$ such that $0 \neq\|h-I d\|_{C^{1}}<\eta$ ( $\eta$ will be defined later). Let $\pi_{k}: B_{r}^{k}(p) \rightarrow B_{r}(0) \subset \mathbb{R}^{3}$ be a local coordinate which is isometry and the derivative of $\pi_{k}$ sends $e_{k}^{s}, e_{k}^{c}, e_{k}^{u}$ to the canonical basis of $\mathbb{R}^{3}$. Let $\xi: \mathbb{R}^{3} \rightarrow \mathbb{R}^{3}$, $\xi_{r}(x):=\frac{1}{r} x$ be the homothety of ratio $\frac{1}{r}$, then we define $\varphi_{k, r}(x):=\xi_{r} \circ \pi_{k}(x)$ and like in section 3.1 we construct $h_{k, r}: \mathbb{T}^{3} \rightarrow \mathbb{T}^{3}$ as follows:

$$
h_{k, r}(w)= \begin{cases}w & \text { if } w \notin B_{r}^{k}(p) \\ \varphi_{k, r}^{-1} \circ h \circ \varphi_{k, r}(w) & , \text { if } w \in B_{r}^{k}(p)\end{cases}
$$

Now the idea is to consider the arcs of diffeomorphisms $f_{k, r}:=f_{k} \circ h_{k, r}$ and show that for some positive $r$ and all large $k, h_{k, r}$ is partially hyperbolic with positive central Lyapunov exponent almost everywhere.

We need to guarantee that these arcs are composed of partially hyperbolic diffeomorphisms. 
Even knowing that the set of partially hyperbolic diffeomorphisms is open in $C^{1}$ topology, we do not know the "size" of this set. To construct our desired examples in Theorems C and E we need the sequence $\left\{h_{k}\right\}$ to be "far from" Id, so it is not obvious that the composition $f_{k, r}=f_{k} \circ h_{k, r}$ is partially hyperbolic. However, in our case this is not a serious issue. As $k$ grows, the domination between invariant sub bundles of $f_{k}$ is getting better and the expansion and contraction of respectively expanding and contracting bundles increase. We observe that when the domination between bundles is bigger, one can take wider invariant cones in the definition of partial hyperbolicity by cones (see section 3.3.1). So it is reasonable to expect that we can do bigger perturbations of $f_{k}$ and still remain in the partially hyperbolic diffeomorphisms set.

Lemma 3.3.1. Let $\left\{f_{k}\right\}$ be the sequence of linear partially hyperbolic automorphisms defined before and $0<r<1$. There exist $\eta>0$ and $K_{0}$ such that if $h: B_{1}(0) \rightarrow$ $B_{1}(0)$ is a diffeomorphism satisfying $\|h-\mathrm{Id}\|_{C^{1}}<\eta$ and equal to the identity on a neighborhood of the boundary of $B_{1}(0)$ then, for $k \geq k_{0}, f_{k} \circ h_{k, r}$ is absolutely partially hyperbolic.

Proof. To make the reading easier we do the technical part needed to prove this lemma in section 3.3.1. In that section for any linear partially hyperbolic diffeomorphism $f$ we estimate the size of the $C^{1}$-neighborhood of $f$ inside the set of absolutely partially hyperbolic diffeomorphisms. Here we are dealing with a sequence $f_{k}$ and the claim is that a same estimate for the size of neighborhood works for all large enough $k$.

Now by Remark 3.3.5 the size of permitted perturbation (i.e the number $\varepsilon$ ) in Lemma 3.3.4 depends increasingly on the ratio $\Theta_{k}:=\min \left\{\frac{\left|\lambda_{k}^{u}\right|}{\left|\lambda_{k}^{c}\right|}, \frac{\left|\lambda_{k}^{c}\right|}{\left|\lambda_{k}^{s}\right|}\right\}$. When $k$ grows this ratio also grows. So we take the same $\varepsilon$ for all $f_{k}$. We should emphasize that the size of permitted perturbation is measured in the distance corresponding to the adapted metric of $f_{k}$.

So let $\varepsilon$ be as above and take any $\eta \leq \varepsilon$. We have $\left\|\xi_{r}^{-1} \circ h \circ \xi_{r}-I d\right\|_{C^{1}} \leq$ $r\|h-I d\|_{C^{1}} \leq\|h-I d\|_{C^{1}} \leq \varepsilon$. By definition of adapted metric for each $f_{k}$ we have that $D \pi_{k}$ preserves norms and angles and consequently the distance (adapted norm corresponding to $\left.f_{k}\right)$ between $\left(\pi_{k} \circ \xi_{r}\right)^{-1} \circ h \circ\left(\pi_{k} \circ \xi_{r}\right)$ and the identity is also less 
than $\varepsilon$ and we can apply Lemma 3.3.4 taking $g:=h_{k, r}$.

By the lemma above it follows that $f_{k, r}$ is partially hyperbolic for large enough $k$. Also, since the same family of invariants cones works for both $f_{k, r}$ and $f_{k}$, the angle between the new center bundle and $E_{f_{k, r}}^{u}$ is uniformly bounded. That is, the norm of the projection of $E_{f_{k, r}}^{c}$ over $E_{f_{k, r}}^{u}$ parallel to the new center bundle is uniformly bounded.

Now let $n_{r}(k)$ be the least positive integer for which $\left(f_{k}\right)^{n_{r}(k)}\left(B_{r}^{k}(p)\right) \cap B_{r}^{k}(p) \neq \emptyset$. Then we have

$$
\sigma_{f_{k, r}}^{c}-\log \lambda_{k}^{c} \geq m\left(B_{r}^{k}(p)\right) \cdot\left(-I(h)-C_{k} \alpha_{k}^{n_{r}(k)}\right)
$$

where

$$
\alpha_{k}=\frac{\lambda^{c}}{\lambda^{u}}, \text { and } C_{k}=\max _{x \in B_{r}^{k}} \frac{h_{r, k}^{c}}{h_{r, k}^{u}} \cdot \max _{x \in B_{r}^{k}}\left\|\operatorname{Proj}_{u}\left(e_{c}\right)\right\| .
$$

As $\max _{x \in B_{r}^{k}}\left\|\operatorname{Proj}_{u}\left(e_{c}\right)\right\|$ is uniformly bounded, it follows that $C_{k}$ is uniformly bounded, say $C_{k}<D, \forall k$. Thus, since $n_{r}(k) \geq 2$ for all $k$, we get:

$$
\sigma_{f_{k, r}}^{c}-\log \lambda_{k}^{c} \geq m\left(B_{r}^{k}(p)\right) \cdot\left(-I(h)-D \alpha_{k}^{2}\right)
$$

Observe that $\alpha_{k} \rightarrow 0$ when $k \rightarrow \infty$. So, for large values of $k$ we have

$$
-I\left(h_{k}\right)-D \alpha_{k}^{2} \geq \frac{-I(h)}{2}
$$

which implies

$$
\sigma_{f_{k, r}}^{c}-\log \lambda_{k}^{c} \geq-m\left(B_{r}^{k}(p)\right) \cdot \frac{I(h)}{2} \rightarrow-m(B(r, p)) \frac{I(h)}{2}>0
$$

Observe that the volume appearing in the above equations is the euclidean volume on the torus and as $k$ is large enough the volume of $B_{r}^{k}(p)$ is close to $B_{r}(0)$. Thus since $\log \lambda_{k}^{c} \rightarrow 0$, for large values of $k$ we get

$$
\sigma_{f_{k, r}}^{c}-\log \lambda_{k}^{c}>-\log \lambda_{k}^{c} \Rightarrow \sigma_{f_{k, r}}^{c}>0
$$

Here we conclude the proof of Theorem C. We have obtained $f_{k, r}$ homotopic to Anosov such that the average of central Lyapunov exponent is positive. Indeed, $H_{k}:[0,1] \times \mathbb{T}^{3} \rightarrow \mathbb{T}^{3}, H_{k}(s, x)=f_{k} \circ h_{k, s r}$ is an isotopy between $f_{k}$ and $f_{k, r}$. 
By continuity we conclude that there exists an open subset of volume preserving diffeomorphisms $U$ containing $f_{k, r}$ such that for any $g \in U$ we have $\sigma_{g}^{c}>0$. By Theorem 2.1.6 we conclude that all $g \in U$ are ergodic and so the central Lyapunov exponent of almost every point is positive.

Now we prove Theorem E. Again from the continuity of $\sigma^{c}$, there is some $0<$ $r_{0}<r$ for which $\sigma_{f_{k, r_{0}}}^{c}=0$. That is, we found a partially hyperbolic diffeomorphism $g:=f_{k, r_{0}}: \mathbb{T}^{3} \rightarrow \mathbb{T}^{3}$, homotopic to an Anosov diffeomorphism and with $\sigma_{g}^{c}=0$. Using again Theorem 2.1.6 we obtain the following:

Corollary 3.3.2. The diffeomorphism g obtained above has zero central Lyapunov exponent almost everywhere.

Proof. Indeed, if $g$ is ergodic then the Lyapunov exponents are constant almost everywhere. So we have $\lambda_{g}^{c}=\sigma_{g}^{c}=0$ almost everywhere. In the other case, that is, if $g$ is not ergodic, then by Theorem 2.1.6 (third item) we have that $\lambda_{g}^{c}=0$ almost everywhere.

To finish the proof we note that, since $g$ is homotopic to a linear Anosov automorphism, it follows from [19] that all central leaves are homeomorphic to $\mathbb{R}$.

Remark 3.3.3. Until this point we have not proved that the diffeomorphism $g$ constructed above with zero center exponent is ergodic because we don't need to use ergodicity to obtain zero exponent almost everywhere due to Theorem 2.1.6. However, we will use the absolute continuity of the center-stable foliation to prove later that this example is indeed ergodic.

\subsubsection{Size of perturbation among absolutely partially hyper- bolic diffeomorphisms}

In this section we develop the technicalities used to prove Lemma 3.3.1. The idea is to find an estimative for the size of the $C^{1}$-neighborhood around a linear partially hyperbolic automorphism inside the set of absolutely partially hyperbolic diffeomorphisms. In order to do so, we use the characterization of absolutely partially hyperbolic diffeomorphisms by invariant cones families (see section 2.1 in the preliminaries). 
Given a partially hyperbolic diffeomorphism $f: \mathbb{T}^{3} \rightarrow \mathbb{T}^{3}$ with invariant splitting

$$
T M=E^{s} \oplus E^{c} \oplus E^{u}
$$

there is an adapted inner product (and then an adapted norm) with respect to which the splitting is orthogonal (see [64]). Thus, given $\beta>0$ we can define standard families of cones centered on the fiber bundles $E^{\tau}(x)$ with angle $\beta, C^{\tau}(x, \beta), \tau=$ $s, c, u, c s, c u$.

Consider $f: M \rightarrow M$ a partially hyperbolic diffeomorphism. By using the adapted norm $\|\cdot\|$, the invariant splitting

$$
T M=E^{s} \oplus E^{c} \oplus E^{u}
$$

is an orthogonal splitting, and there exist numbers

$$
0<\lambda_{1} \leq \mu_{1}<\lambda_{2} \leq \mu_{2}<\lambda_{3} \leq \mu_{3}, \quad \mu_{1}<1, \quad \lambda_{3}>1
$$

for which

$$
\begin{aligned}
& \lambda_{1} \leq\left\|D f(x) \mid E^{s}(x)\right\| \leq \mu_{1} \\
& \lambda_{2} \leq\left\|D f(x) \mid E^{c}(x)\right\| \leq \mu_{2} \\
& \lambda_{3} \leq\left\|D f(x) \mid E^{u}(x)\right\| \leq \mu_{3} .
\end{aligned}
$$

For the linear case, that is, if $f$ is linear, we can get some more precise and interesting conclusions regarding the relation of the constants of (2.1) and (2.2) and the Lyapunov exponents of the function. In what follows, for a linear partially hyperbolic diffeomorphism, we find an explicit relation between the angle of the invariant cones families and the ratio of domination between unstable, stable and central bundles.

Consider $f: \mathbb{T}^{3} \rightarrow \mathbb{T}^{3}$ a linear, volume preserving, partially hyperbolic diffeomorphism of $\mathbb{T}^{3}$. Denote by $\lambda^{s}, \lambda^{c}, \lambda^{u}$ its eigenvalues, where

$$
\left|\lambda^{s}\right|<\left|\lambda^{c}\right|<\left|\lambda^{u}\right|, \quad\left|\lambda^{s}\right|<1<\left|\lambda^{u}\right|
$$

Put

$$
\Theta:=\min \left\{\frac{\left|\lambda^{c}\right|}{\left|\lambda^{s}\right|}, \frac{\left|\lambda^{u}\right|}{\left|\lambda^{c}\right|}\right\}
$$


Then we can choose a constant $\beta>0$ such that

$$
1<(1+\beta)^{2}<\Theta
$$

Therefore, by the definition of $\beta$, we have

$$
(1+\beta)\left|\lambda^{s}\right|<\frac{\left|\lambda^{c}\right|}{1+\beta}<(1+\beta)\left|\lambda^{c}\right|, \quad(1+\beta)\left|\lambda^{s}\right|<1<\frac{\left|\lambda^{u}\right|}{1+\beta} .
$$

Consequently we can find constants $\mu_{1}, \lambda_{2}, \mu_{2}, \lambda_{3}$ such that

$$
(1+\beta)\left|\lambda^{s}\right|<\mu_{1}<\lambda_{2}<\frac{\left|\lambda^{c}\right|}{1+\beta}<(1+\beta)\left|\lambda^{c}\right|<\mu_{2}<\lambda_{3}<\frac{\left|\lambda^{u}\right|}{1+\beta}, \quad \mu_{1}<1<\lambda_{3}
$$

Now, it is straightforward to verify that with the constants defined by (3.6) and (3.7), the families of stable, unstable, center-stable and center-unstable cones satisfies (2.1) and (2.2). For example, if we take $v=v_{s}+v_{c u} \in C^{c u}(x, \beta)$ then

$$
\left\|D f(x) v_{s}||=\left|\lambda^{s}\right||| v_{s}|| \leq \beta\left|\lambda^{s}\right||| v_{c u}||<\beta\left|\lambda^{c}\right||| v_{c u}|| \leq \beta\right\| D f(x) v_{c u} \|
$$

that is

$$
D f(x)\left(C^{c u}(x, \beta)\right) \subset C^{c u}(f(x), \beta) .
$$

Furthermore,

$$
\|D f(x) v\|^{2} \geq\left\|D f(x) v_{c u}\right\|^{2} \geq\left|\lambda^{c}\right|^{2}\left\|v_{c u}\right\|^{2} .
$$

But by (3.7) we know that $\left|\lambda^{c}\right|>(1+\beta) \lambda_{2}$. So,

$$
\begin{gathered}
\|D f(x) v\|^{2}>(1+\beta)^{2}\left(\lambda_{2}\right)^{2}\left\|v_{c u}\right\|^{2} \geq\left(\lambda_{2}\right)^{2}\left(\left\|v_{c u}\right\|^{2}+\beta^{2}\left\|v_{c u}\right\|^{2}\right) \geq \lambda_{2}^{2}\left(\left\|v_{c u}\right\|^{2}+\left\|v_{s}\right\|^{2}\right)=\left(\lambda_{2}\|v\|\right)^{2} \\
\Rightarrow\|D f(x) v\|>\lambda_{2}\|v\| .
\end{gathered}
$$

The argument for the other cones is similar.

Lemma 3.3.4. Let $f: \mathbb{T}^{3} \rightarrow \mathbb{T}^{3}$ be a linear partially hyperbolic diffeomorphism, volume preserving, with eigenvalues $\lambda^{s}, \lambda^{c}, \lambda^{u}$, where $\left|\lambda^{s}\right|<\left|\lambda^{c}\right|<\left|\lambda^{u}\right|$. Then, there 
is a constant $\varepsilon>0, \varepsilon$ depending only on $\Theta:=\min \left\{\frac{\left|\lambda^{u}\right|}{\left|\lambda^{c}\right|}, \frac{\left|\lambda^{c}\right|}{\left|\lambda^{s}\right|}\right\}$, such that, for every diffeomorphism $g: \mathbb{T}^{3} \rightarrow \mathbb{T}^{3}$ with $\| g$ - Id $\|_{C^{1}}<\varepsilon$ (adapted norm corresponding to $f)$, the composition $f \circ g$ is an absolutely partially hyperbolic diffeomorphism.

Remark 3.3.5. It is easy to see from the proof that $\varepsilon$ is an increasing function of $\Theta$. Bigger $\Theta$ permits bigger perturbations. However we emphasize that the distance between $g$ and identity is considered in the adapted metric.

Proof. Let $f$ be as in the statement and denote the invariant splitting of $f$ by

$$
T_{x} M=E^{s}(x) \oplus E^{c}(x) \oplus E^{u}(x) .
$$

Consider the adapted norm $\|\cdot\|$ with respect to which the invariant splitting is orthogonal. Now, since $f$ is a linear partially hyperbolic diffeomorphisms we can choose constants

$$
\begin{gathered}
0<\lambda_{1} \leq \mu_{1}<\lambda_{2} \leq \mu_{2}<\lambda_{3} \leq \mu_{3} \\
\mu_{1}<1<\lambda_{3}
\end{gathered}
$$

and a real value $\beta>0$ as in (3.6) and (3.7).

For $v \in M$ we can write $v=v_{s}+v_{c}+v_{u}$ with $v_{\tau} \in E^{\tau}(x), \tau=s, c, u$.

- If $v \in C^{u}(x, \beta)$ then $\left\|v_{c s}\right\| \leq \beta\left\|v_{u}\right\|$, where $v_{c s}=v_{s}+v_{c}$. Thus,

$$
\begin{gathered}
\left\|D f(x) v_{c s}\right\|<\mu_{2}\left\|D f^{-1} \circ D f(x) v_{c s}\right\|=\mu_{2}\left\|v_{c s}\right\| \leq \mu_{2} \beta\left\|v_{u}\right\|<\mu_{2} \beta\left(\lambda_{3}\right)^{-1}\left\|D f(x) v_{u}\right\| . \\
\Rightarrow D f(x) v \in C^{u}\left(f(x),\left(\mu_{2} / \lambda_{3}\right) \cdot \beta\right) .
\end{gathered}
$$

- If $v \in C^{c u}(x, \beta)$ then $\left\|v_{s}\right\| \leq \beta\left\|v_{c u}\right\|$ where $v_{c u}=v_{c}+v_{u}$. Thus,

$$
\begin{gathered}
\left\|D f(x) v_{s}\right\|<\mu_{1}\left\|D f^{-1} \circ D f(x) v_{s}\right\|=\mu_{1}\left\|v_{s}\right\| \leq \mu_{1} \beta\left\|v_{c u}\right\|<\mu_{1} \beta\left(\lambda_{2}\right)^{-1}|| D f(x) v_{c u} \| \\
\Rightarrow D f(x) C^{c u}(x, \beta) \subset C^{u}\left(f(x),\left(\mu_{1} / \lambda_{2}\right) \cdot \beta\right) .
\end{gathered}
$$


- If $v \in E^{s}(x)$ then $\left\|v_{c u}\right\| \leq \beta\left\|v_{s}\right\|$. Thus,

$$
\begin{gathered}
\left\|D f^{-1}(x) v_{c u}\right\|<\lambda_{2}^{-1}\left\|D f \circ D f^{-1}(x) v_{c u}\right\|=\lambda_{2}^{-1}\left\|v_{c u}\right\| \leq \lambda_{2}^{-1} \beta\left\|v_{s}\right\|<\lambda_{2}^{-1} \beta \mu_{1}\left\|D f^{-1}(x) v_{s}\right\| \\
\Rightarrow D f^{-1}(x) C^{s}(x, \beta) \subset C^{s}\left(f^{-1}(x),\left(\mu_{1} / \lambda_{2}\right) \cdot \beta\right) .
\end{gathered}
$$

- If $v \in E^{c s}(x)$ then $\left\|v_{u}\right\| \leq \beta\left\|v_{c s}\right\|$. Thus,

$$
\begin{aligned}
\left\|D f^{-1}(x) v_{u}\right\| & <\lambda_{3}^{-1}|| D f \circ D f^{-1}(x) v_{u}\left\|=\lambda_{3}^{-1}\right\| v_{u}\left\|\leq \lambda_{3}^{-1} \beta\right\| v_{c s}\left\|<\lambda_{3}^{-1} \beta \mu_{2}|| D f^{-1}(x) v_{c s}\right\| \\
& \Rightarrow D f^{-1}(x) C^{c s}(x, \beta) \subset C^{c s}\left(f^{-1}(x),\left(\mu_{2} / \lambda_{3}\right) \cdot \beta\right) .
\end{aligned}
$$

Define

$$
\gamma:=\max \left\{\frac{\mu_{2}}{\lambda_{3}}, \frac{\mu_{1}}{\lambda_{2}}\right\}<1
$$

So we have

$$
\begin{aligned}
D f^{-1}(x)\left(\mathcal{C}^{\tau}(x, \beta)\right) & \subset \mathcal{C}^{\tau}\left(f^{-1}(x), \gamma \cdot \beta\right), \tau=s, c s \\
D f(x)\left(\mathcal{C}^{\Psi}(x, \beta)\right) & \subset \mathcal{C}^{\Psi}(f(x), \gamma \cdot \beta), \Psi=u, c u
\end{aligned}
$$

Observe that by (3.6) and (3.7), $\beta$ and $\gamma$ depend only on the ratios $\left|\lambda^{u}\right| /\left|\lambda^{c}\right|,\left|\lambda^{c}\right| /\left|\lambda^{s}\right|$.

Now, since the invariant splitting is constant, we can take an $\varepsilon>0$ depending only on the ratios $\left|\lambda^{u}\right| /\left|\lambda^{c}\right|,\left|\lambda^{c}\right| /\left|\lambda^{s}\right|$ such that, if $\|g-I d\|_{C^{1}}<\varepsilon$ then

$$
\begin{gathered}
D g(x) C^{\tau}(x, \gamma \cdot \beta) \subset C^{\tau}(g(x), \beta), \tau=u, c u \\
D g^{-1}(x) C^{\Psi}(x, \gamma \cdot \beta) \subset C^{\Psi}\left(g^{-1}(x), \beta\right), \Psi=s, c s
\end{gathered}
$$

and

$$
\frac{l}{L}>\gamma, \quad L<\frac{1}{\mu_{1}}, \quad \frac{1}{\lambda_{3}}<l
$$

with

$$
l\|v\| \leq\|D g(x) v\| \leq L\|v\| .
$$




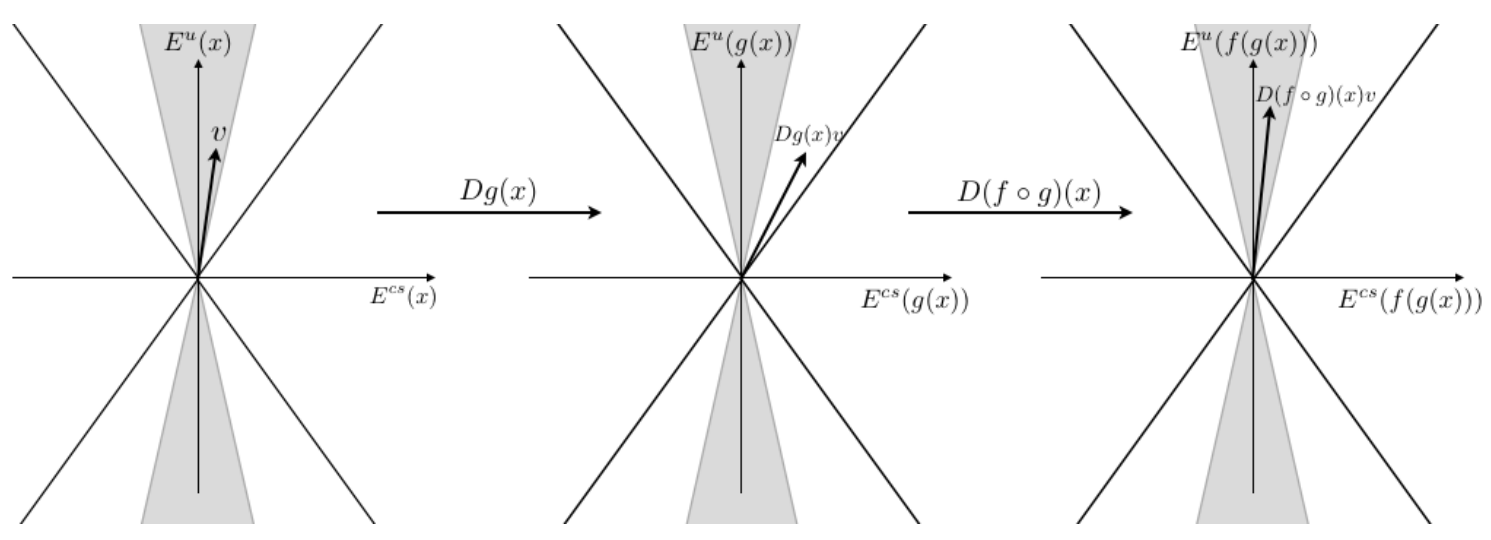

Figure 3.1: ( [44]) The grey cones denote the cones with angle $\gamma \cdot \beta$ and the wider ones are the cones with angle $\beta$.

Thus we have (see figure 4.1)

$$
\begin{gathered}
D(f \circ g)(x)\left(C^{\tau}(x, \gamma \cdot \beta)\right) \subset C^{\tau}(f(g(x)), \gamma \cdot \beta), \tau=u, c u \\
D(f \circ g)^{-1}(x)\left(C^{\Psi}(x, \beta)\right) \subset C^{\Psi}\left(g^{-1}\left(f^{-1}(x)\right), \beta\right), \Psi=s, c s .
\end{gathered}
$$

Now, we need to show uniform contraction and expansion on these families.

- If $v \in C^{u}(x, \gamma \cdot \beta)$ then

$$
\|D(f \circ g)(x) v\| \geq \lambda_{3}\|D g(x) v\| \geq \lambda_{3} \cdot l \cdot\|v\| .
$$

- If $v \in C^{c s}(x, \beta)$ then

$$
\left\|D(f \circ g)^{-1}(x) v\right\| \geq L^{-1}|| D f^{-1}(x) v\left\|>L^{-1} \cdot \mu_{2}^{-1}\right\| v \| .
$$

- If $v \in C^{c u}(x, \beta \cdot \gamma)$ then

$$
\|D(f \circ g)(x) v\| \geq \lambda_{2}\|D g(x) v\| \geq \lambda_{2} \cdot l \cdot\|v\| .
$$


- If $v \in C^{s}(x, \beta)$ then

$$
\left\|D(f \circ g)^{-1}(x) v\right\| \geq L^{-1}\left\|D f^{-1}(x) v\right\|>L^{-1} \cdot \mu_{1}^{-1}\|v\|
$$

Furthermore

$$
0<L \cdot \mu_{1}<l \cdot \lambda_{2} \leq L \cdot \mu_{2}<l \cdot \lambda_{3}
$$

and

$$
L \cdot \mu_{1}<1, l \cdot \lambda_{3}>1
$$

so that $f \circ g$ is absolutely partially hyperbolic as we claimed.

\subsection{Ergodicity of the pathological example}

To prove that the pathological example constructed in section 3.3 has zero center Lyapunov exponent almost everywhere we didn't use ergodicity as observed in remark 3.3.3 but in fact we can easily prove that the example is ergodic using a Hopf argument for the unstable and center-stable foliations.

The first observation is the following lemma.

Lemma 3.4.1. Let $f \in \mathcal{P} \mathcal{H}_{m}^{2}\left(\mathbb{T}^{3}\right)$ be a DA with linearization $A$ such that $\lambda_{A}^{c}<0$. Assume that the center stable foliation of $f$ is absolutely continuous. Then $f^{n}$ is ergodic for every $n \in \mathbb{Z}$.

Proof. Assume $f$ is not ergodic. Then by Theorem 2.1.6 there is $h: \mathbb{T}^{3} \rightarrow \mathbb{T}^{3}$ homeomorphism maping strong leaves to strong leaves and conjugating $f$ and $A$. Consider $\varphi$ any continuous function of $\mathbb{T}^{3}$.

Consider two points $x, y \in \mathbb{T}^{3}$ in the same center manifold of $f$. Then $h(x)$ and $h(y)$ are in the same center manifold of $A$. Since $h$ is a homeomophism $h(x)$ and $h(y)$ are different and, since the center manifold is contracting, they have the same 
forward Birkhoff limit for the continuous function $\varphi \circ h^{-1}$ with respect to $A$, that is

$$
\begin{aligned}
\left(\varphi \circ h^{-1}\right)_{A,+}(h(x)) & =\lim _{n \rightarrow+\infty} \frac{1}{n} \sum_{i=0}^{n-1} \varphi \circ h^{-1}\left(A^{i}(h(x))\right) \\
& =\lim _{n \rightarrow+\infty} \frac{1}{n} \sum_{i=0}^{n-1} \varphi \circ h^{-1}\left(A^{i}(h(y))\right)=\left(\varphi \circ h^{-1}\right)_{A,+}(h(y)) .
\end{aligned}
$$

By conjugacy we have $h^{-1} \circ A^{i} \circ h=f$ thus

$$
\left(\varphi \circ h^{-1}\right)_{f,+}(x)=\left(\varphi \circ h^{-1}\right)_{f,+}(y)
$$

Since the same happens for the stable manifold, i.e, two points in the same stable manifold have the same forward Birkhoff average, then every two points in the same center-stable manifold have the same $\varphi_{f,+}$.

Let $A$ be the set of points $x$ for which $\varphi_{f,+}(x)=\varphi_{f,-}(x)$. By Birkhoff's theorem, $m(A)=1$. By absolute continuity of the stable foliation for almost every $x$ we have that $A \cap F_{f}^{c s}(x)$ has total measure in the leaf. Every point $x \in A \cap F_{f}^{c s}(x) \subset A$ has the same forward and backward limit which we denote by $\Phi$. Let $A^{u}(x)$ be the $u$-saturation of $A \cap F_{f}^{c s}(x)$ and set

$$
B=A \cap A^{u}(x)
$$

Then $B$ has full measure and if $a \in B$ then $\varphi_{f,-}(a)=\varphi_{f,-}\left(\mathcal{F}^{u}(a) \cap A\right)=\Phi$, but $\varphi_{f,-}(a)=\varphi_{f,+}(a)$ so that for every $a \in B$ the forward and backward limits are equal to $\Phi$.

This implies that $f$ is ergodic contradicting the hypothesis.

Since $f^{n}$ satisfies the same conditions of the theorem for all $n \geq 0$ then $f^{n}$ is ergodic for every integer $n$.

Corollary 3.4.2. Let $f \in \mathcal{P H}_{m}^{\infty}\left(\mathbb{T}^{3}\right)$ be the example constructed in Theorem E. Then $f$ is ergodic.

Proof. By the construction of the example we know that for any $x \in \mathbb{T}^{3}, \mathcal{F}_{f}^{c s}(x)=$ 
$\mathcal{F}_{A}^{c s}(x)$, which is a foliation by parallel planes, thus absolutely continuous. Then by Lemma 3.4.1 $f$ is ergodic.

Question 3.4.3. Is $f$ essentially accessible? Is $f$ Kolmogorov?

Although we expect the answer to be positive, we could not solve these questions which remain open. 


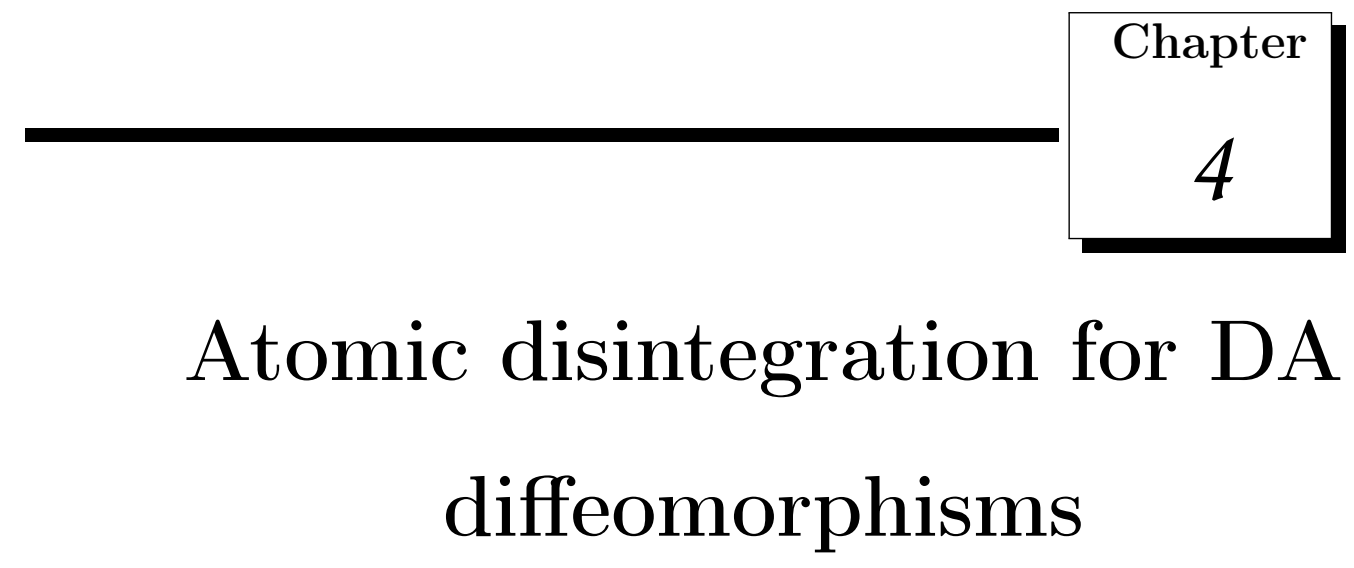

Let $(M, \mu, \mathcal{B})$ be a probability space, where $M$ is a compact metric space, $\mu$ is a probability measure and $\mathcal{B}$ is the Borelian $\sigma$-algebra. Given a partition $\mathcal{P}$ of $M$ by measurable subsets, the quotient space can be equipped with a natural measure and in general it may be a singular measure space, i.e the measurable subsets have just measure zero or one. For example, this is the case for the partition of $\mathbb{T}^{2}$ into orbits of irrational flow (Kronecker flow). There is an opposite situation which is the case of measurable partitions. The word measurable here should be understood as countably generated, as in Definition 2.2.4. In this case, the measure $\mu$ can be disintegrated into probability (conditional) measures. See Section 2.2 for details. A foliation is a particular case of (not necessarily measurable) partition. It is well worth recalling that the quotient space of a foliated space may not be Hausdorff. This is the case in the example of Sullivan [58] where all the leaves are compact however, their size goes to infinity. Indeed, any foliation with compact leaves is measurable (see Proposition 2.2.5).

In smooth ergodic theory, one of the natural partitions to be studied is the partition into leaves of invariant foliations of a dynamical system. Some celebrated examples are the stable and unstable foliations of (uniformly) hyperbolic dynamics. The partition into global stable or unstable leaves is non-measurable and there are deep results where techniques are developed to find measurable partitions "subordi- 
nate" to the global leaves (see for instance [33]).

Non-measurability "typically" comes with some non trivial topological properties of the leaves. For instance this is the case of stable and unstable leaves of a transitive Anosov diffeomorphism. These foliations are minimal, i.e every leaf is dense. Although the notion of minimality seems to be in the opposite direction to the measurability, in this chapter we find minimal yet measurable foliations.

The results of this chapter mainly focus on the understanding of disintegration of volume measure along the central invariant foliation of partially hyperbolic diffeomorphisms.

Theorem F. There exists an open subset $U$ of volume preserving partially hyperbolic diffeomorphisms on $\mathbb{T}^{3}$ such that for any $g \in U$ the central foliation is minimal yet measurable (with respect to Lebesgue measure) as a partition.

The proof of the above result is based on a careful study of disintegration of Lebesgue measure along the central foliation of diffeomorphisms in $U$ which were constructed in [44] (see Chapter 3). We consider the example of the above theorem as a nice application of a dynamical systems argument to topological and geometrical measure theoretic properties of foliations.

In fact Theorem $\mathrm{F}$ is a consequence of the construction made in Chapter 3 together with two general results (Theorem B and Theorem D) on the disintegration of Lebesgue measure along the central foliation of ergodic Derived from Anosov diffeomorphisms on $\mathbb{T}^{3}$ which we restate below.

Theorem B. ( [45]) Let $f \in \mathcal{P} \mathcal{H}_{m}^{2}\left(\mathbb{T}^{3}\right)$ be an ergodic DA diffeomorphism on $\mathbb{T}^{3}$. If the volume measure $m$ has atomic disintegration on the center leaves, then the disintegration is mono-atomic.

In principle the disintegration of the Lebesgue measure along central foliation of a DA diffeomorphism can be non-atomic even if it is singular with respect to Lebesgue (see [60]). This makes the study of Derived from Anosov (even Anosov, seen as partially hyperbolic systems) diffeomorphisms interesting from the point of view of geometric measure theory.

Another relevant comment is that for a general partially hyperbolic diffeomorphism (not Derived from Anosov), atomic disintegration along central foliation does not imply mono-atomicity. There exist partially hyperbolic diffeomorphisms where 
the disintegration of Lebesgue measure is atomic with finite (strictly larger than one) atoms. There are also systems with conditional measures with infinitely many atoms. See section 4.1 for some examples and discussion.

In the proof of Theorem $\mathrm{F}$ we take advantage of the fact that the sign of the central Lyapunov exponent for any $f \in U$ is opposite to the sign of the central Lyapunov exponent of its linearization (see section 2.1 for definition) $f_{*}$. Then, the following Theorem concludes the proof of Theorem F.

Theorem D. ( [45]) Let $f \in \mathcal{P H}_{m}^{2}\left(\mathbb{T}^{3}\right)$ be homotopic to an Anosov linear automorphism $A: \mathbb{T}^{3} \rightarrow \mathbb{T}^{3}$. If

$$
\lambda_{f}^{c}(x) \lambda_{A}^{c}<0
$$

for Lebesgue almost every point $x \in \mathbb{T}^{3}$, then volume has atomic disintegration on $\mathcal{F}_{f}^{c}$, in fact the disintegration is mono-atomic.

Remark 4.0.4. It is worth to note that the previous theorem gives complete information of the disintegration of volume along center foliation having only the information of opposite signs of the center exponent of $f$ and A. That is, alone the opposite asymptotic behavior of the center foliations of $f$ and $A$ implies atomicity and at the same time minimality of this foliation.

\subsection{Disintegration of volume along invariant folia- tions}

Let $f$ be a partially hyperbolic diffeomorphism with

$$
T M=E^{s} \oplus E^{c} \oplus E^{u} .
$$

It is well known that the stable and the unstable foliations of $f$ are absolutely continuous (see section 2.2). Absolute continuity of stable and unstable foliations imply that a set of full volume on $M$ must intersect almost every leaf of $\mathcal{F}^{s}$ (or $\mathcal{F}^{u}$ ) in a set of full Lebesgue measure of the leaf. Although the absolute continuity of $\mathcal{F}^{s}$ and $\mathcal{F}^{u}$ are mandatory for a (general) $C^{2}$ partially hyperbolic diffeomorphism, this is not the case for the center foliation $\mathcal{F}^{c}$ (it is not even true that there will exist such 
a foliation, but by [7] and [8] for all absolutely partially hyperbolic diffeomorphisms of $\mathbb{T}^{3}$ the center foliation exists as discussed in section 2.1). The center foliation might not be absolutely continuous, at least this is not expected to happen for diffeomorphisms which preserve volume in general (see [56], [27], [18], [57], [4]). For many examples (some of them described below) the center foliation has atomic disintegration. For a general partially hyperbolic diffeomorphism the geometric structure of the support of the conditional disintegrated measures is not clear.

There exist essentially three known categories of partially hyperbolic diffeomorphisms on three-dimensional manifolds (see conjecture of Pujals in [6] and new results of Hammerlindl-Potrie [21] for a better discussion), roughly speaking they are:

- $D A$ diffeomorphisms (see Definition 2.1.2);

- skew-product-type partially hyperbolic diffeomorphisms, and

- perturbations of time-one maps of Anosov flows.

We will briefly describe some disintegration results obtained for these three classes.

\subsubsection{Disintegration of volume along center foliation}

Let $B:=A \times I d$ where $A:=\left(\begin{array}{ll}2 & 1 \\ 1 & 1\end{array}\right)$. Then arbitrarily close to $B: \mathbb{T}^{3} \rightarrow \mathbb{T}^{3}$ there is an open set of partially hyperbolic diffeomorphisms $g$ such that $g$ is ergodic and there is an equivariant fibration $\pi: \mathbb{T}^{3} \rightarrow \mathbb{T}^{2}$ such that the fibers are circles, $\pi \circ g=B \circ \pi$. Moreover $g$ has positive central Lyapunov exponent, hence the central foliation is not absolutely continuous. In Ruelle and Wilkinson's paper [55], we see that there exist $S \subset \mathbb{T}^{3}$ of full volume and $k \in \mathbb{N}$ such that $S$ meets every leaf in exactly $k$ points. In Shub and Wilkinson's example [57] the fibers of the fibration are invariant under the action of a finite non-trivial group and consequently in their example the number of atoms cannot be one.

For the perturbation of a time-one map of the geodesic flow on a closed negatively curved surface (which is an Anosov flow), it was shown by A. Avila, M. Viana and A. Wilkinson [3] that $\mathcal{F}^{c}$ either has atomic disintegration or is absolutely continuous. We emphasize that the key property for the diffeomorphisms near time-one maps of 
Anosov flows is that they are partially hyperbolic and with all center leaves fixed by the dynamics. This implies that in the atomic case, the disintegrated measures do have countably (infinitely) many atoms. Indeed, if $f$ is such a partially hyperbolic diffeomorphism $f\left(\mathcal{F}^{c}(x)\right)=\mathcal{F}^{c}(x)$ and for any atom $a, f^{n}(a), n \in \mathbb{Z}$ are atoms of the disintegration of volume along unbounded leaves of $\mathcal{F}^{c}$.

For a large class of skew-product diffeomorphisms, Avila-Viana and Wilkinson announced that they can prove an analogous result, i.e atomicity versus absolute continuity.

It is interesting to emphasize that (conservative) Derived from Anosov (DA) diffeomorphisms on $\mathbb{T}^{3}$ show a feature that is not shared with any other known partially hyperbolic diffeomorphisms in dimension three, they admit all three disintegration of volume on the center leaf, namely: Lebesgue, atomic, and, by a recent result of $\mathrm{R}$. Varão [60], they can also have a disintegration which is neither Lebesgue nor atomic. More precisely, R. Varão [60] showed that there exist Anosov diffeomorphisms with non-absolutely continuous center foliation which do not have atomic disintegration.

Here we show a new behavior for DA diffeomorphisms (which are not Anosov) on $\mathbb{T}^{3}$, that is the existence of mono-atomic disintegration (Theorem D). This behavior can be verified for an open class of diffeomorphisms found by Ponce-Tahzibi in [44].

We mention that the examples of non-absolutely continuous weak foliation of Anosov diffeomorphisms was known by Saghin-Xia [56] and A. Gogolev [18]. BaravieraBonatti [4] also exhibited non-absolutely continuous central foliation for partially hyperbolic diffeomorphisms close to Anosov geodesic flows. We are introducing examples of non-Anosov diffeomorphisms with non-absolutely continuous central foliation and prove atomic disintegration. The novelty in our example is that, the sign of central Lyapunov exponent of a partially hyperbolic diffeomorphism is opposite to the sign of central Lyapunov exponent of its linearization. It is not known whether the disintegration of the Lebesgue measure can be atomic in the case of Anosov diffeomorphisms.

\subsubsection{Comments on the results of this chapter}

The diffeomorphisms in $U$ are constructed homotopic to Anosov linear diffeomorphisms on $\mathbb{T}^{3}$. By a result of A. Hammerlindl [19], partially hyperbolic diffeomorphisms on $\mathbb{T}^{3}$ are leaf-conjugated to their linearization and hence the central foliation of any $g \in U$ is minimal. 
As we mentioned before, any $g \in U$ satisfies the hypothesis of Theorem $\mathrm{D}$ and consequently the disintegration of Lebesgue measure along the central foliation is mono-atomic. The mono-atomicity implies that the partition into central leaves is equivalent to the partition into single points and hence it is measurable. So, we get minimal and measurable foliation.

We remark that the technical part of the proof of Theorem D is similar to the arguments of [55].

For the arguments we use in this chapter the one-dimensional center hypothesis is essential. Indeed, for the two-dimensional center case the disintegration of the volume measure is not well understood.

Question 4.1.1. Let $f: \mathbb{T}^{4} \rightarrow \mathbb{T}^{4}$ be a $C^{2}$ volume-preserving partially hyperbolic diffeomorphism with $\operatorname{dim}\left(E^{c}\right)=2$. What are the possibilities for the disintegration of $m$ along the center leaves?

\subsection{Proof of Theorem B}

Let $f$ be a partially hyperbolic DA diffeomorphism, then by Franks and Manning (see section 2.1.1) we know that $f$ is semi-conjugated to its linearization by a map $h: \mathbb{T}^{3} \rightarrow \mathbb{T}^{3}, h \circ f=A \circ h$. It follows from [59] (see section 2.1.1) that $\mathcal{F}^{c}(A)=$ $h\left(\mathcal{F}^{c}(f)\right)$. Moreover, there exists a constant $K \in \mathbb{R}$ such that if $\tilde{h}: \mathbb{R}^{3} \rightarrow \mathbb{R}^{3}$ denotes the lift of $h$ to $\mathbb{R}^{3}$ we have $\|\tilde{h}(x)-x\| \leq K$ for all $x \in \mathbb{R}^{3}$.

Definition 4.2.1. A foliation $\mathcal{F}$ defined on a Riemannian manifold $M$ is quasiisometric if the lift $\widetilde{\mathcal{F}}$ of $\mathcal{F}$ to the universal cover of $M$ has the following property: There exist positive constants $Q, Q^{\prime}$ such that for all $x, y$ in a common leaf of $\widetilde{\mathcal{F}}$ we have

$$
d_{\widetilde{\mathcal{F}}}(x, y) \leq Q\|x-y\|+Q^{\prime}
$$

where $d_{\widetilde{\mathcal{F}}}$ denotes the Riemannian metric on $\widetilde{\mathcal{F}}$ and $\|x-y\|$ is the distance on the universal cover.

Remark 4.2.2. The leaves of foliations under consideration are $C^{1}$ and tangent to a continuous subbundle and consequently after a change of constants we can assume $Q^{\prime}=0$ in the above definition. 
For absolutely partially hyperbolic diffeomorphisms on $\mathbb{T}^{3}$ the stable, unstable and central foliations are quasi-isometric in the universal covering $\mathbb{R}^{3}[8,19]$. First we prove Theorem B.

Proof of Theorem B. Let $h: \mathbb{T}^{3} \rightarrow \mathbb{T}^{3}$ be the semi-conjugacy between $f$ and its linearization $A$, hence $h \circ f=A \circ h$. We can assume that $A$ has two eigenvalues larger than one, otherwise we work with $f^{-1}$.

Let $\left\{R_{i}\right\}$ be a Markov partition for $A$, and define $\hat{R}_{i}:=h^{-1}\left(R_{i}\right)$. We claim that

$$
m\left(\bigcup_{i} \operatorname{int} \hat{R}_{i}\right)=1
$$

First look at the center direction of $A$. For simplicity we consider the center direction as a vertical foliation. This means that the rectangle $R_{i}$ has two types of boundaries, the one coming from the extremes of the center foliation and the lateral ones. We call $\partial_{c} R_{i}$ the boundary coming from these extremes of the center foliation, i.e

$$
\partial_{c} R_{i}=\bigcup_{x \in R_{i}} \partial\left(\mathcal{F}_{x}^{c} \cap R_{i}\right)
$$

Since $h$ takes center leaves to center leaves, we conclude that the respective boundary for the $\hat{R}_{i}$ sets is $\partial_{c} \hat{R}_{i}=h^{-1}\left(\partial_{c} R_{i}\right)$, and since $\bigcup_{i} \partial_{c} R_{i}$ is an $A$-invariant set, $\bigcup_{i} \partial_{c} \hat{R}_{i}$ is a forward $f$-invariant set. By the ergodicity of $f$ it follows that $\bigcup_{i} \partial_{c} \hat{R}_{i}$ has zero or full measure. Since the volume of the interior cannot be zero, then the volume of $\bigcup_{i} \partial_{c} \hat{R}_{i}$ cannot be one. Therefore it has zero measure. Since $f$ is dynamicaly coherent and $h$ sends each of the invariant foliations (center-stable and center-unstable) to the respective invariant foliations of $A$, that implies that the lateral boundaries of the $\hat{R}_{i}$ are inside a finite number of center-stable and center-unstable leaves of $f$, hence they also have zero volume. We have avoided the boundary points as there may exist ambiguity to which element of partition these points belong.

By (4.1) we can consider the partition $\hat{\mathcal{P}}=\left\{\mathcal{F}_{R}^{c}(x): x \in \hat{R}_{i}\right.$ for some $\left.i\right\}$ where $\mathcal{F}_{R}^{c}(x)$ denotes the connected component of $\mathcal{F}_{f}^{c}(x) \cap \hat{R}(x)$ which contains $x$ in its interior. Thus we can consider the Rokhlin disintegration of volume on the partition 
$\hat{\mathcal{P}}$. Denote this system of measures by $\left\{m_{x}\right\}$, so that each $m_{x}$ is supported in $\mathcal{F}_{f}^{c}(x)$.

Lemma 4.2.3. There is a natural number $\alpha_{0} \in \mathbb{N}$, such that for almost every point $x, \mathcal{F}_{R}^{c}(x)$ contains exactly $\alpha_{0}$ atoms.

Proof. The semi-conjugacy $h$ sends center leaves of $f$ to center leaves of $A$. Also, the points of the interior of the $\hat{R}_{i}$ satisfy that $f\left(\mathcal{F}_{R}^{c}(x)\right) \supset \mathcal{F}_{R}^{c}(f(x))$, which just comes from the Markov property of the rectangles $R_{i}$. We claim that

$$
f_{*} m_{x} \leq m_{f(x)}
$$

on $\mathcal{F}_{R}^{c}(f(x))$.

In fact, since $f$ preserves volume if we normalize the measures $f_{*} m_{x}$ on $f\left(\mathcal{F}_{R}^{c}(x)\right) \cap$ $\mathcal{F}_{R}^{c}(f(x))$ they become a disintegration of volume on $f\left(\mathcal{F}_{R}^{c}(x)\right) \cap \mathcal{F}_{R}^{c}(f(x))$, but $m_{f(x)}$ are also a disintegration of volume on $f\left(\mathcal{F}_{R}^{c}(x)\right) \cap \mathcal{F}_{R}^{c}(f(x))$. Hence, $f_{*} m_{x}=$ $f_{*} m_{x}\left(f\left(\mathcal{F}_{R}^{c}(x)\right) \cap \mathcal{F}_{R}^{c}(f(x))\right) m_{f(x)}$. And because the normalization constant $f_{*} m_{x}\left(f\left(\mathcal{F}_{R}^{c}(x)\right) \cap\right.$ $\left.\mathcal{F}_{R}^{c}(f(x))\right)$ is smaller or equal to one we get the above inequality.

Given any $\delta \geq 0$ consider the set $A_{\delta}=\left\{x \in \mathbb{T}^{3} \mid m_{x}(\{x\})>\delta\right\}$, that is, the set of atoms with weight at least $\delta$. If $x \in A_{\delta}$ then

$$
\delta<m_{x}(\{x\})=f_{*} m_{x}(\{f(x)\}) \leq m_{f(x)}(\{f(x)\}) .
$$

Thus $f\left(A_{\delta}\right) \subset A_{\delta}$, and by the ergodicity of $f$ we have that $m\left(A_{\delta}\right)$ is zero or one, for each $\delta \geq 0$. Note that $m\left(A_{0}\right)=1$ and $m\left(A_{1}\right)=0$. Let $\delta_{0}$ be the critical point for which $m\left(A_{\delta}\right)$ changes value, i.e, $\delta_{0}=\sup \left\{\delta: m\left(A_{\delta}\right)=1\right\}$. This means that all the atoms have weight $\delta_{0}$. And due to the assumption of atomic disintegration, the value of $\delta_{0}$ has to be a strictly positive number. Since $m_{x}$ is a probability we have an $\alpha_{0}:=1 / \delta_{0}$ number of atoms as claimed.

Lemma 4.2.4. There are only finitely many atoms on almost every center leaf.

Proof. Suppose we have infinitely many atoms on each center leaf. Let $\beta \in \mathbb{R}$ be a large number (for instance much bigger than $K Q$ where $K$ is the distance between $h$ 
and the identity map and $Q$ is the quasi isometric constant in the definition 4.2.1). Since we have a finite number of $\hat{R}_{i}$, from the previous Lemma, we know that there is a number $\tau \in \mathbb{R}$ for which every center segment of size smaller then $\beta$ must contain at most $\tau$ atoms. But, since on each center leaf there are infinity many atoms, take a segment of leaf big enough so that it contains more then $\tau$ atoms. Iterate this segment backwards and it will eventually be smaller than $\beta$ but containing more than $\tau$ atoms. Indeed,

$$
\begin{gathered}
h \circ f^{-n}=A^{-n} \circ h \\
\left\|h\left(f^{-n}(x)\right)-h\left(f^{-n}(y)\right)\right\|=\left\|A^{-n}(h(x))-A^{-n}(h(y))\right\| \\
\leq e^{-n \lambda^{w u}(A)}\|h(x)-h(y)\|
\end{gathered}
$$

As $h$ is at a distance $K$ to identity we have

$$
\left\|f^{-n}(x)-f^{-n}(y)\right\| \leq e^{-n \lambda^{w u}(A)}\|h(x)-h(y)\|+K \leq \frac{\beta}{Q}
$$

So, finally by quasi isometric property

$$
d_{c}\left(f^{-n}(x), f^{-n}(y)\right) \leq \beta .
$$

The contradiction above implies that the number of atoms can not be infinite and now we proceed as in the previous case.

Lemma 4.2.1. The disintegration of the Lebesgue measure along the central leaves is mono-atomic, i.e there is just one atom per leaf.

Proof. We have a finite number of atoms on each center leaf and since the center foliation is an oriented foliation we may talk about the first atom. If the function $f$ preserves orientation along the center direction then the set of first atoms of all generic leaves is an invariant set with positive measure, therefore it has full measure. If $f$ reverses orientation, then the set of first and last atoms of all generic leaves is an invariant set with positive measure, therefore it has full measure. This means that almost every leaf has exactly one atom or almost every leaf has exactly two 
atoms.

Assume that almost every center leaf has exactly two atoms. Since the set of first atoms is invariant by $f^{2}$ then $f^{2}$ is not ergodic. By Theorem 2.1.6 there exist a homeomorphism $h: \mathbb{T}^{3} \rightarrow \mathbb{T}^{3}$ mapping center (stable, unstable) leaves of $f^{2}$ onto center (stable, unstable) leaves of $A^{2}$ and conjugating $f^{2}$ and its linearization $A^{2}: \mathbb{T}^{3} \rightarrow \mathbb{T}^{3}$, that is

$$
A^{2} \circ h=h \circ f^{2}
$$

It is well known that if we require $h$ to be homotopic to identity then such conjugacy is unique. Thus the homeomorphism $h$ also conjugates $f$ and $A$,

$$
A \circ h=h \circ f .
$$

Consider $A_{k}$ the set of points $x$ such that the distance between two atoms on the central leaf passing through $x$ is less than $k$. for large $k$ the measure of $A_{k}$ is positive and almost every point of $A_{k}$ returns to it infinitely many time. Take such $x \in A_{k}$ and $a, b \in \mathcal{F}^{c}(x)$ the two atoms of the disintegration. By invariance $f^{n_{i}}(a), f^{n_{i}}(b)$ are atoms on $\mathcal{F}^{c}\left(f^{n_{i}}(x)\right)$. Taking $f^{n_{i}}(x) \in A_{k}$, on the one hand we have that $d\left(f^{n_{i}}(a), f^{n_{i}}(b)\right) \leq k$. On the other hand

$$
d\left(A^{n_{i}}(h(a)), A^{n_{i}}(h(b))\right)=d\left(h\left(f^{n_{i}}(a)\right), h\left(f^{n_{i}}(b)\right)\right) .
$$

As $h$ is injective and the left hand side of the above equation goes to infinity, we get a contradiction.

The above Lemma concludes the proof of the Theorem B.

Question 4.2.5. Is there any ergodic invariant measure $\mu$ with atomic disintegration and having more than one atom on leaves?

We note once again that since the work of Ponce-Tahzibi [44] (see chapter 3) assures that the set of DA satisfying the hypothesis of the next theorem is nonempty, we prove that these diffeomorphisms have atomic disintegration. 


\subsection{A Glimpse of Pesin Theory}

Before presenting the proof of Theorem $D$, we recall some basic notions of Pesin theory. Let $f: \mathbb{T}^{3} \rightarrow \mathbb{T}^{3}$ a partially hyperbolic diffeomorphism with splitting

$$
T M=E^{s} \oplus E^{c} \oplus E^{u} .
$$

Call $\Lambda$ the set of regular points (see [62], [5]) of $f$, that is, the set of points $x \in \mathbb{T}^{3}$ for which in particular the Lyapunov exponents are well defined. Then, for each $x \in \Lambda$ we define the Pesin-stable manifold of $f$ at $x$ as the set

$$
W^{s}(x)=\left\{y: \limsup _{n \rightarrow \infty} \frac{1}{n} \log d\left(f^{n}(x), f^{n}(y)\right)<0\right\}
$$

The Pesin-stable manifold is an immersed sub manifold of $\mathbb{T}^{3}$. Similarly we define the Pesin-unstable manifold at $x, W^{u}(x)$, using $f^{-1}$ instead of $f$ in the definition.

It is clear that for a partially hyperbolic diffeomorphism $W^{s}(x)$ contains the stable leaf $\mathcal{F}^{s}(x)$. In Theorem D we can assume that the central Lyapunov exponent of $f$ is negative (otherwise we work with $f^{-1}$ ) and consequently the Pesin-stable manifolds of $f$ are two-dimensional. By $\mathcal{W}^{c}(x)$ we denote the intersection of the Pesin stable manifold $W^{s}(x)$ of $x$ with the center leaf $\mathcal{F}_{f}^{c}(x)$ of $x$. These manifolds depend only measurably on the base point $x$, as it is proved in Pesin theory. However, there is a filtration of the set of regular points by Pesin blocks: $\Lambda=\bigcup_{l \in \mathbb{N}} \Lambda_{l}$ such that each $\Lambda_{l}$ is a closed (not necessarily invariant) subset and $x \rightarrow \mathcal{W}^{c}(x)$ varies continuously on each $\Lambda_{l}$ (see [5] for more properties and structure of these sets).

A key property used in the proof of Theorem D is the uniform contraction locally around points in a Pesin block:

Lemma 4.3.1. (see chapter 7 of [5]) There exists $C>0, \lambda<1$ and $r_{l}>0$ such that for any $x \in \Lambda_{l}$ (Pesin block)

$$
d\left(f^{n}(x), f^{n}(y)\right) \leq C \lambda^{n} d(x, y)
$$

for any $y \in W_{r_{l}}^{s}(x)$. Here $W^{s}$ stands for the Pesin stable manifold and $d$ is the induced distance. 


\subsection{Proof of Theorems D and F}

Proof of Theorem D. First of all, as remarked before, we can assume that the center Lyapunov exponent of the linearization $A$ is positive and the center Lyapunov exponent of $f$ is negative for Lebesgue almost every point. To begin, we prove that the size of the weak stable manifolds $\mathcal{W}^{c}(x)$ is uniformly bounded from above for $x$ belonging to the regular set. In particular this enables us to prove that the partition $(\bmod -0)$ by $\mathcal{W}^{c}(x)$ is a measurable partition.

Lemma 4.4.1. The size of $\left\{\mathcal{W}^{c}(x)\right\}_{\left\{x: \lambda^{c}(x)<0\right\}}$ is uniformly bounded from above for $x \in \Lambda$. More precisely, the image of $\mathcal{W}^{c}(x)$ by $h$ is a unique point.

Proof. Let $\tilde{f}: \mathbb{R}^{3} \rightarrow \mathbb{R}^{3}$ and $\tilde{A}: \mathbb{R}^{3} \rightarrow \mathbb{R}^{3}$ denote the lifts of $f$ and $A$ respectively and $\tilde{h}: \mathbb{R}^{3} \rightarrow \mathbb{R}^{3}$ the lift of the semi-conjugacy $h$ between $f$ and $A$. Consider $\gamma \subset \widetilde{\mathcal{W}}^{c}(x)$, where $\widetilde{\mathcal{W}}^{c}(x)$ is the lift of $\mathcal{W}^{c}(x)$. Thus, $\gamma$ is inside the intersection of the center manifold of $\tilde{f}$ and the Pesin-stable manifold of $\tilde{f}$ passing through $x$.

Let us show that $\tilde{h}$ collapses $\widetilde{\mathcal{W}}^{c}(x)$ to a single point. If we prove that, it clearly follows (from the bounded distance of $h$ to identity) that, the size of $\mathcal{W}^{c}(x)$ is uniformly bounded. Suppose by contradiction that $\tilde{h}(\gamma)$ has more than one point. By semi-conjugacy $\tilde{h}\left(\tilde{f}^{n}(\gamma)\right)=\tilde{A}^{n}(\tilde{h}(\gamma))$. As $\tilde{h}(\gamma)$ is a subset of weak unstable foliation of $\tilde{A}$ for large $n$ the size of $\tilde{A}^{n}(\tilde{h}(\gamma))$ is large. On the other hand, $\gamma$ is in the Pesin stable manifold of $f$ and consequently for large $n$, the size of $\tilde{f}^{n}(\gamma)$ is very small. As $\|\tilde{h}-\mathrm{Id}\| \leq K$ we conclude that for large $n$ the size of $\tilde{h}\left(\tilde{f}^{n}(\gamma)\right)$ can not be very big. This contradiction completes the proof.

Corollary 4.4.2. The family $\left\{\mathcal{W}^{c}(x)\right\}_{\left\{x: \lambda^{c}(x)<0\right\}}$ forms a measurable partition.

This corollary uses the same idea of the proof of Proposition 2.2.5. However, that proposition is proved for continuous foliations and we adapt the proof for the Pesin measurable lamination.

Proof. First of all we consider a new partition $\left\{\overline{\mathcal{W}^{c}(x)}\right\}$ whose elements are the closure of the elements $\mathcal{W}^{c}(x)$, that is, $\overline{\mathcal{W}^{c}(x)}$ is a bounded length center segment 
together with its extreme points. Since $h$ collapses the manifolds $\mathcal{W}^{c}(x)$ of $f$ into points, two different elements $\mathcal{W}^{c}(x)$ and $\mathcal{W}^{c}(y)$ cannot have a common endpoint, so that $\left\{\overline{\mathcal{W}^{c}(x)}\right\}$ is indeed a partition with compact elements. Let us prove that it is indeed a measurable partition.

Let $\left\{x_{j}\right\}_{j \in \mathbb{N}}$ be a countable dense set of $M=\mathbb{T}^{3}$. For each $x_{j}$ and $k, l \in \mathbb{N}$ we define $C_{l}\left(x_{j}, k\right)$ to be the union of $\mathcal{W}^{c}(y), y \in \Lambda_{l}$ such that $\mathcal{W}^{c}(y)$ intersects the closed ball $B\left(x_{j}, \frac{1}{k}\right)$. By continuity of $\mathcal{W}^{c}(\cdot)$ on $\Lambda_{l}$ we conclude that $C_{l}\left(x_{j}, k\right)$ is closed and consequently measurable. Indeed, if $y_{n} \in C_{l}\left(x_{j}, k\right)$ converges to $y$ then $y \in \Lambda_{l}$ and moreover $\mathcal{W}^{c}(y)$ intersects the closure of $B\left(x_{j}, \frac{1}{k}\right)$.

Now, we need to separate two weak stable manifolds by means of some $C_{l}\left(x_{j}, k\right)$. Taking two elements $\mathcal{W}^{c}(a)$ and $\mathcal{W}^{c}(b)$ there exists $l \in \mathbb{N}$ such that $a, b \in \Lambda_{l}$ and it is enough to take small enough $k$ and some $x_{j}$ such that $C_{l}\left(x_{j}, k\right)$ contains $\mathcal{W}^{c}(a)$ and not $\mathcal{W}^{c}(b)$. It is easy to see that for each $x \in \Lambda$ we have $\mathcal{W}^{c}(x)=\bigcap_{k, l, j} C_{l}\left(x_{j}, k\right)^{*}$ where $C_{l}\left(x_{j}, k\right)^{*}$ is either $C_{l}\left(x_{j}, k\right)$ or $\mathbb{T}^{3} \backslash C_{l}\left(x_{j}, k\right)$.

Now, observe that if $y$ is an extreme point of $\mathcal{W}^{c}(x)$ then $y$ cannot have negative center Lyapunov exponent, otherwise it would be in the interior of $\mathcal{W}^{c}(x)$. So, the set of extreme points of the elements $\overline{\mathcal{W}^{c}(x)}$ is inside the set of points with non negative center Lyapunov exponent, and therefore it has zero measure. Thus, removing such points, the measurability of the partition $\left\{\overline{\mathcal{W}^{c}(x)}\right\}$ implies the measurability of $\left\{\mathcal{W}^{c}(x)\right\}$, concluding the proof of the corollary.

Lemma 4.4.3. Disintegration of volume on the measurable partition $\left\{\mathcal{W}^{c}(x)\right\}$ is atomic.

Proof. Let $\mathcal{W}^{c}(x, r)$ denote a ball inside $\mathcal{W}^{c}(x)$ of radius $r$. By elementary Pesin theory we know that $\bigcup_{l \geq 1} \Lambda_{l}$ has full Lebesgue measure where $\Lambda_{l}$ are Pesin blocks. Here we use the fact that for $x \in \Lambda_{l}$ the size of Pesin invariant manifolds is bounded from below by $r_{l}>0$. For sufficiently large $l$ we have $m\left(\Lambda_{l}\right)>1 / 2$.

As $\mathcal{W}^{c}(x)$ is measurable we consider the disintegration of Lebesgue measure into conditional probability measures $m_{x}$. Since $\mathcal{W}^{c}$ is $f$-invariant and $f$ preserves 
volume, Corollary 2.2.3 implies

$$
m_{f(x)}=f_{*} m_{x}
$$

Let us define

$$
J:=\bigcup_{m_{x}\left(\mathcal{W}^{c}(x) \cap \Lambda_{l}\right) \geq 1 / 2} \mathcal{W}^{c}(x) .
$$

It is clear that $m(J)>0$. Consider the set $B \subset J$ that consists of points that return to $J$ infinitely many times. By Poincaré recurrence $m(B)=m(J)$. Let $F: B \rightarrow B$ be the first return map of $f$.

Observe that if $x \in B$ and $F(x)=f^{n}(x)$ then $m_{f^{n}(x)}\left(\mathcal{W}^{c}\left(f^{n}(x)\right) \cap \Lambda_{l}\right) \geq 1 / 2$. By invariance of $\mathcal{W}^{c}$ we have $f^{n}\left(\mathcal{W}^{c}(x)\right)=\mathcal{W}^{c}\left(f^{n}(x)\right)$. Using the definition of $J$, $F(y)=f^{n}(y)$ for any $y \in \mathcal{W}^{c}(x)$ and consequently $F\left(\mathcal{W}^{c}(x)\right)=\mathcal{W}^{c}(F(x))$. As $F$ preserves volume, Corollary 2.2.3 implies $F_{*} m_{x}=m_{F(x)}$.

As the size of $\mathcal{W}^{c}(x)$ is bounded from above for almost all $x$, we can take $k$ such that every $\mathcal{W}^{c}(x)$ can be covered by at most $k$ balls of radius $r_{l} / 2$. So for all $x \in B$ we can choose a ball $B_{x}^{c}$ of radius $r_{l} / 2$ such that $m_{x}\left(B_{x}^{c}\right) \geq \frac{1}{2 k}$ and $B_{x}^{c} \cap \Lambda_{l} \neq \emptyset$.

Now let $B_{n, x}^{c}=F^{n}\left(B_{F^{-n}(x)}^{c}\right)$ and observe that $m_{x}\left(B_{n, x}^{c}\right)=m_{F^{-n}(x)}\left(B_{F^{-n}(x)}^{c}\right) \geq$ $\frac{1}{2 k}$. As the size of the stable manifold of $x \in \Lambda_{l}$ is larger than $r_{l}$ we conclude that $B_{n, x}^{c}$ is completely inside the Pesin stable manifold and consequently the diameter of $B_{n, x}^{c}$ goes to zero with an uniform rate due to Lemma 4.3.1.

We have proved that for all $x \in B$ there is a sequence of sets whose diameters decrease uniformly and these sets have $m_{x}$-measure uniformly positive. By taking a subsequence if necessary we can assume that these sets accumulate on a point. Then, any neighborhood of such point has positive measure uniformly bounded from below, this implies that this point is an atom. Since the set of atoms is an invariant set with positive volume, the ergodicity of $f$ implies that this set has full volume, hence $m_{x}$ is a sum of Dirac measures.

Since the previous lemma gives us atomicity, we can apply Theorem B to get one atom per leaf. 
Finally let us use Theorems D and Theorem C (which we proved in chapter 3) to complete the proof of Theorem F.

Proof of Theorem $\boldsymbol{F}$. By Theorem $\mathrm{C}$ there exists an open set $U$ such that any $g \in$ $U$ is volume preserving partially hyperbolic with Anosov linearization $g_{*}$ satisfying the following property: The central Lyapunov exponent of $g$ is negative but the central bundle of $g_{*}$ is expanding. So, $g$ satisfies the hypothesis of Theorem D and consequently there is a subset of full Lebesgue measure which intersects almost all center leaves in a unique point. This means that the partition into central leaves is measure theoretically equivalent to the partition into single points of $\mathbb{T}^{3}$. This implies measurability of the foliation. The minimality of the central foliation follows from the minimality of central foliation of $g_{*}$ and the leaf conjugacy between $g$ and $g_{*}$, as we commented in Section 2.1.

Remark 4.4.4. We remark that all the examples of atomic disintegration known before had compact or at least bounded leaves. The famous paradoxical example given by Katok [37], is a foliation of $N=(0,1) \times[0,1]$ by bounded pieces of analytic curves which intersects a full measure subset of $N$ in exactly one point. Our example shows a higher degree of pathology since each leaf is dense in the whole manifold $M=\mathbb{T}^{3}$ but still it intersects a full measure set in exactly one point. 


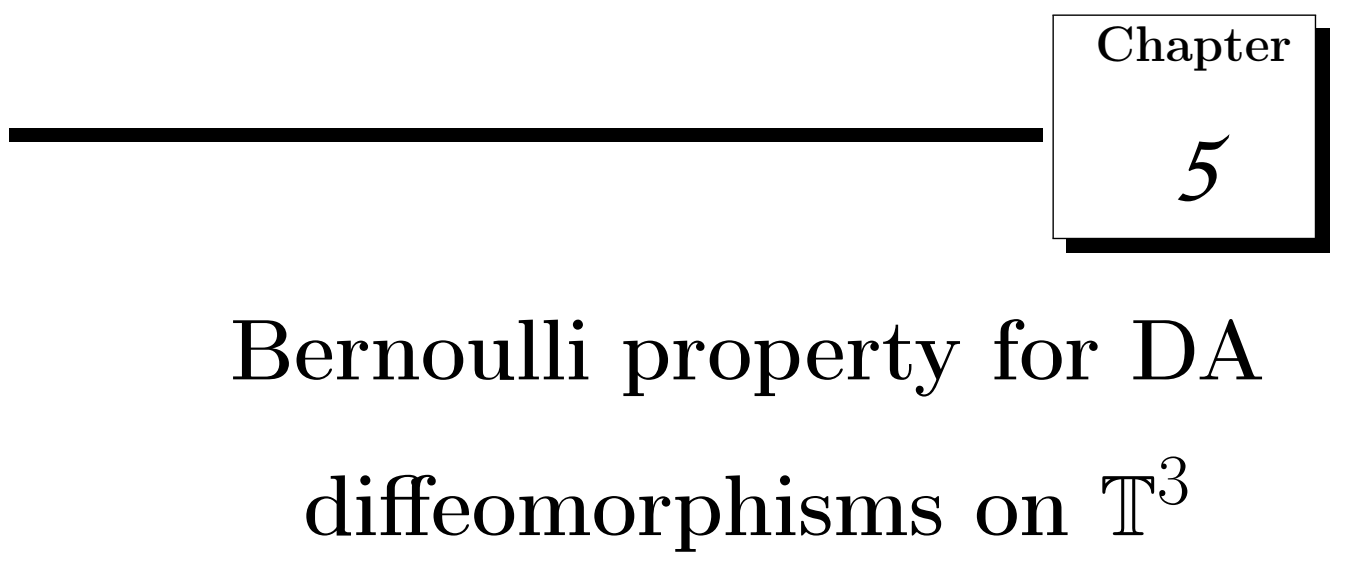

In section 2.3 we briefly contextualized the Bernoulli and the Kolmogorov properties as ergodic hierarchy classes of dynamical systems. Before going to the results we give a small historical context of the relationship between the equivalence of these two properties and the theory of smooth dynamical systems. We refer to [42] for a more complete account of how the theory has developed.

Bernoulli shifts are easy to define and constitute the most chaotic class of measure preserving transformations. One of the questions which interested ergodic theorists in the past was the classification of such systems. The first step in this direction was given by Kolmogorov [32] when he proved that not all Bernoulli shifts were equivalent. However, it was only in 1970 that a complete classification of Bernoulli systems was given by two remarkable works of D. Ornstein [38], [40], who showed that Bernoulli shifts are completely classified by their entropy. The proof of Ornstein's isomorphism theorem uses a property called finitely determined (which is equivalent to Very Weak Bernoulli property, see section 5.1) which is derived from independence. It was observed later that the hyperbolic structure of some smooth dynamical systems could be used to verify the finitely determined property (or the Very Weak Bernoulli property) and thus the Bernoulli property.

The chaotic nature of system with hyperbolic structure became more evident when D. Ornstein and B. Weiss [14] proved that geodesic flows on negatively curved 
surfaces were Bernoulli. The method developed in [14] sets the basis used in almost all the other results proving Bernoulli property from some hyperbolic structure. In this method, Ornstein and Weiss used in an essential way two properties satisfied by the geodesic flow: the Kolmogorov property and the absolute continuity of the unstable and stable foliations. With these two properties, they were able to show that partitions by elements with smooth boundaries had the property called Very Weak Bernoulli, which is the key tool to constructed Bernoulli partitions by "approximation".

At this point in the history, abstract ergodic theory and smooth ergodic theory were being quickly developed and the relationships between the structures of the smooth and the abstract setting were arising. For example, in [14] Ornstein and Weiss used in an essential way the Kolmogorov property to obtain Bernoulli property for the geodesic flow. However, the relations between the Kolmogorov and the Bernoulli property were still a little bit obscure. In the same year D. Ornstein [41] constructed a Kolmogorov but non Bernoullian automorphism. The automorphism constructed by D. Ornstein is not a natural one, and has no smooth structure. It was only in 1982 that $\mathrm{S}$. Kalikow proved that the $\left(T, T^{-1}\right)$-transformation (see section 2.3, example 2.3.7) was a Kolmogorov but not a Bernoullian system, and A. Katok [30] gave the first example of a $C^{\infty}$ transformation in a smooth manifold which is Kolmogorov but not Bernoulli. Katok's example can be constructed to be partially hyperbolic. A few years before the examples of Kalikow and Katok, Y. Pesin [62] showed that for non-uniformly hyperbolic smooth dynamical systems with non-zero Lyapunov exponents, the Kolmogorov and the Bernoulli property are equivalent (see section 2.3). The natural question at this point is what happens if the system admits zero center Lyapunov exponent?

Observe that if one wants to study the possible equivalence of Kolmogorov and Bernoulli properties for smooth dynamical systems on 3-manifolds this is an essential question.

Since the equivalence of Kolmogorov and Bernoulli properties is true for Anosov diffeomorphisms, the class of DA diffeomorphisms would be the natural starting point to try to extend the equivalence of Kolmogorov and Bernoulli properties. The presence of a center direction that is not necessarily expanding or contracting as the other directions, is clearly the main obstruction. Not only this direction has a different metric behavior, but also it is not necessarily absolutely continuous, as 
are the other directions. On the other hand, for DA diffeomorphism we have the Franks-Manning semi-conjugacy (see subsection 2.1.1) which allows us to compare the center behavior of the DA with the center behavior of the linearization for large subsets of $\mathbb{T}^{3}$.

We recall that all along the thesis we work with absolutely partially hyperbolic diffeomorphisms only, so that, for the sake of simplicity, we omit the term "absolutely".

In this chapter we prove that for DA diffeomorphisms in $\mathcal{P H}_{m}^{2}\left(\mathbb{T}^{3}\right)$ the Kolmogorov and the Bernoulli properties are equivalent.

Theorem A. ([46]) Let $f \in \mathcal{P H}_{m}^{2}\left(\mathbb{T}^{3}\right)$ be homotopic to a linear Anosov. If $f$ is Kolmogorov, then $f$ is Bernoulli.

The proof follows the same lines of [14] and [5], thus we made an effort to keep the same notation and names of some objects (such as rectangles, leafwise intersection, etc.) that we need to use in the proof.

We remark that the results of this section are true for higher dimensional cases provided quasi-isometry (see definition 4.2.1) of strong foliations and one dimensional center direction.

Theorem G. Let $f \in \mathcal{P} \mathcal{H}_{m}^{2}\left(\mathbb{T}^{n}\right)$ with one dimensional center bundle, homotopic to an Anosov automorphism A, and with quasi-isometric strong foliations. Then, if $f$ is Kolmogorov, $f$ is Bernoulli.

Theorem A answers, for some specific cases, the following question raised by K. Burns during the problems section at the congress Recent Progress in Dynamics 2006, held at the Clay Mathematics Institute (see [23] section 11).

Question 5.0.5 ( [23]). Let $M$ be a compact Riemannian manifold and let $f: M \rightarrow$ $M$ be a $C^{2}$ volume-preserving partially hyperbolic diffeomorphism. Assume $f$ is (essentially) accessible and center bunched. Is $f$ Bernoulli?

As remarked in [23] the expected answer was negative, but all known examples of Kolmogorov and not Bernoulli diffeomorphisms in the literature are not of this type. For $M=\mathbb{T}^{3}$ and $f$ with non-compact center leaves we show that the answer is positive. 


\subsection{The Very Weak Benoulli property}

Let $(X, \nu)$ be a Lebesgue space. We will always assume that the measures we consider are normalized, that is, if $A \subset X$ is a measurable subset then by the measure on $A$ we mean the measure $\nu(A)^{-1} \nu$.

Definition 5.1.1. Let $\xi$ be a finite partition of $X$. We say that a property holds for $\varepsilon$-almost every element of $\xi$ if the union of those elements for which the property fails has $\nu$-measure at most $\varepsilon$.

In order to define what is called a Very Weak Bernoulli (VWB) partition we need to introduce a metric in the space of finite partitions.

Let $\xi_{i}=\left\{A_{1}^{(i)}, \ldots, A_{m}^{(i)}\right\}$ and $\eta_{i}=\left\{B_{1}^{(i)}, \ldots, B_{m}^{(i)}\right\}, 1 \leq i \leq n$, be two sequences of partitions of Lebesgue spaces $(X, \nu)$ and $(Y, \mu)$ respectively. We write

$$
\left\{\xi_{i}\right\}_{1}^{n} \sim\left\{\eta_{i}\right\}_{1}^{n}
$$

if for any $1 \leq k_{i} \leq m$ and $1 \leq i \leq n$,

$$
\nu\left(\bigcap_{i=1}^{n} A_{k_{i}}^{(i)}\right)=\mu\left(\bigcap_{i=1}^{n} B_{k_{i}}^{(i)}\right)
$$

Definition 5.1.2. We say that the distance between $\left\{\xi_{i}\right\}_{1}^{n}$ and $\left\{\eta_{i}\right\}_{1}^{n}$ is less or equal $\varepsilon$, and denote it by

$$
d\left(\left\{\xi_{i}\right\}_{1}^{n},\left\{\eta_{i}\right\}_{1}^{n}\right) \leq \varepsilon
$$

if there exist sequences of partitions $\bar{\xi}_{i}=\left\{\bar{A}_{1}^{(i)}, \ldots, \bar{A}_{m}^{(i)}\right\}$ and $\bar{\eta}_{i}=\left\{\bar{B}_{1}^{(i)}, \ldots, \bar{B}_{m}^{(i)}\right\}$ of a Lebesgue space $(Z, \lambda)$ such that

$$
\left\{\xi_{i}\right\}_{1}^{n} \sim\left\{\bar{\xi}_{i}\right\}_{1}^{n} \text { and }\left\{\eta_{i}\right\}_{1}^{n} \sim\left\{\bar{\eta}_{i}\right\}_{1}^{n}
$$

and

$$
\frac{1}{n} \sum_{i=1}^{n} \sum_{j=1}^{m} \lambda\left(\bar{A}_{j}^{(i)} \triangle \bar{B}_{j}^{(i)}\right) \leq \varepsilon .
$$


Definition 5.1.3. A partition $\xi$ of a Lebesgue space $(X, \nu)$ is called VWB if for any $\varepsilon>0$ there exists $N_{0}=N_{0}(\varepsilon)$ such that for any $N^{\prime}>N \geq N_{0}, n \geq 0$, and $\varepsilon$-almost every element $A \in \bigvee_{k=N}^{N^{\prime}} f^{k} \xi$, we have

$$
d\left(\left\{f^{-i} \xi\right\}_{1}^{n},\left\{f^{-i} \xi \mid A\right\}_{1}^{n}\right) \leq \varepsilon
$$

Let us emphasize that the partition $\xi \mid A$ is considered with respect to the normalized measure.

D. Ornstein proved that the VWB property can be used to obtain Bernoulli property for a system.

Theorem 5.1.4 ([40]). If $\alpha$ is a VWB partition, then the system $\left(X, \bigvee_{-\infty}^{+\infty} f^{-i} \alpha, \mu, f\right)$ is Bernoulli.

Theorem 5.1.5 (see [14], [39]). Let $f$ be an automorphism of a Lebesgue space $(X, \nu)$. Take $\epsilon$ to be the partition of $X$ by points. Assume that there exists a sequence of VWB partitions $\xi_{1} \leq \xi_{2} \leq \ldots$ such that $\xi_{n} \rightarrow \epsilon$. Then $f$ is a Bernoulli automorphism.

Given a sequence of partitions $\left\{\xi_{i}\right\}_{1}^{n}$ of a Lebesgue space $(X, \nu)$, we define the sequence of integer-valued functions $l_{i}(x)$ by the condition $x \in A_{l_{i}(x)}^{(i)}$. We call the sequence of functions $l_{i}(x)$ the name of the sequence of partitions $\left\{\xi_{i}\right\}_{1}^{n}$.

Definition 5.1.6. We say that a transformation $\theta: X \rightarrow Y$ of Lebesgue spaces $(X, \nu)$ and $(Y, \mu)$ is $\varepsilon$-measure preserving if there exists a set $E \subset X$ such that $\nu(E) \leq \varepsilon$ and for every measurable set $A \subset X \backslash E$,

$$
\left|\frac{\mu(\theta(A))}{\nu(A)}-1\right| \leq \varepsilon .
$$

Define the function $e: \mathbb{N} \rightarrow\{0,1\}$ by $e(0)=0$ and $e(n)=1$ for all $n>0$.

The following lemma proved by D. Ornstein and B. Weiss in [14] makes a geometric approach possible to prove the Very Weak Bernoulli property. 
Lemma 5.1.7 (Ornstein-Weiss, [14]). Let $\left\{\xi_{i}\right\}_{1}^{n}$ and $\left\{\eta_{i}\right\}_{1}^{n}$ be two sequences of partitions of Lebesgue spaces $(X, \nu)$ and $(Y, \mu)$ with name functions $l_{i}(x)$ and $m_{i}(x)$ respectively. Assume that there exists an $\varepsilon$-measure preserving transformation $\theta$ : $X \rightarrow Y$, satisfying

$$
\frac{1}{n} \sum_{i=1}^{n} e\left(l_{i}(x)-m_{i}(\theta(x))\right) \leq \varepsilon
$$

for all $x \in X$ with possible exception for a set $E \subset X$ with $\nu(E) \leq \varepsilon$. Then

$$
d\left(\left\{\xi_{i}\right\}_{1}^{n},\left\{\eta_{i}\right\}_{1}^{n}\right) \leq 16 \varepsilon
$$

The proof of Theorem A is based on the construction of a function $\theta$ satisfying the previous lemma. The approach used in [14] and [62] was to construct $\theta$ preserving stable manifolds, so it guarantees that the $f$-orbits of $x$ and $\theta(x)$ cannot be far from each other. In our case, due to the presence of the center manifold, it is not possible to construct $\theta$ preserving the stable manifold, thus we have to work a little bit with the semi-conjugacy to obtain good non-divergence along the center for at least large sets.

\subsection{Kolmogorov property}

As in [14] and in [62] the following consequence of $K$-property (see section 2.3) is used to prove the Bernoulli property.

Lemma 5.2.1 (Ornstein-Weiss, [14]). Let $f$ be a K-automorphism of a Lebesgue space $(Y, \mu), \xi$ a finite partition of $Y$, and $B \subset Y$ a measurable set. Given $\delta>0$, there exists $N_{0}>0$ such that for any $N^{\prime}>N \geq N_{0}$ and $\delta$-almost every element $A \in \bigvee_{k=N}^{N^{\prime}} f^{k} \xi$,

$$
\left|\frac{\mu(A \cap B)}{\mu(A)}-\mu(B)\right| \leq \delta .
$$

This lemma allows us to find the desired almost measure-preserving function $\theta$ (necessary to apply Lemma 5.1.7) just locally and then to extend the function $\theta$ to the whole manifold while keeping it almost measure-preserving. 


\subsection{Proof of Theorem A}

The proof can be divided in a few steps which we list below:

1. Use the Franks-Manning semi-conjugacy to obtain an invariant set of collapsed $\operatorname{arcs} \mathcal{C}$;

2. Prove that either the volume of $\mathcal{C}$ is zero or the disintegration is atomic with 1-atom per leaf;

3. We restrict to $X=\{$ atoms $\}$, or $X=\mathbb{T}^{3} \backslash \mathcal{C}$ and note that in this set, $h$ is a bijection, which allow us to take an arbitrarily large compact set where $h$ is a homeomorphism. This helps us to control divergence of points in the same center foliation when restricted to $X$;

4. Construct the partition $\alpha$ that we want to show that is VWB and the "controlling" partition of rectangles;

5. Use Ornstein-Pesin's method to construct $\theta$ and restrict this $\theta$ to the basin of the volume measure inside a huge compact set;

6. Conclude that the partition is VWB.

\section{Proof of Theorem A.}

Let $f: \mathbb{T}^{3} \rightarrow \mathbb{T}^{3}$ be a $C^{2}$ Kolmogorov partially hyperbolic diffeomorphism homotopic to a linear Anosov diffeomorphism $A: \mathbb{T}^{3} \rightarrow \mathbb{T}^{3}$.

Without loss of generality we can assume that the center Lyapunov exponent $\lambda_{A}^{c}$ of $A$ is negative: $\lambda_{A}^{c}<0$ (otherwise we work with $f^{-1}$ which is homotopic to $A^{-1}$ ). Again by results of J. Franks [16] and A. Manning [34], we have a semi-conjugacy $h: \mathbb{T}^{3} \rightarrow \mathbb{T}^{3}$ between $A$ and $f$ that is:

$$
A \circ h=h \circ f
$$

and that the $C^{0}$ - distance $\|\tilde{h}-\mathrm{Id}\|<K$ is bounded by a finite constant $K>0$, where $\tilde{h}: \mathbb{R}^{3} \rightarrow \mathbb{R}^{3}$ is a lift of $h$. Thus, given any point $x_{0} \in \mathbb{T}^{3}$ the set $h^{-1}\left(\left\{x_{0}\right\}\right)$ is uniformly bounded inside the center foliation. 
By results of R. Ures [59] we know that $h$ takes center leaves of $f$ to center leaves of $A$, and that each set $h^{-1}\left(\left\{x_{0}\right\}\right)$ is a point or a connected arc of center manifold. Given a point $x \in \mathbb{T}^{3}$ define the set $c(x) \subset \mathcal{F}^{c}(x)$ by:

$$
c(x)=h^{-1}(\{h(x)\}) .
$$

By the above discussion, the diameter of $c(x)$ is uniformly bounded in $x$.

Take

$$
\mathcal{C}:=\bigcup_{y \in\left\{x \in \mathbb{T}^{3} \mid c(x) \neq\{x\}\right\}} c(y) .
$$

It is easy to see that $f(\mathcal{C})=\mathcal{C}$, for if $h(a)=h(b)$ then by $(5.1)$ we have

$$
h(f(a))=A(h(a))=A(h(b))=h(f(b))
$$

and if $h(a) \neq h(b)$ then by $(5.1)$ we have

$$
h(f(a))=A(h(a)) \neq A(h(b))=h(f(b)) .
$$

By ergodicity of $f$ it follows that $\mathcal{C}$ has either full or zero volume.

Lemma 5.3.1. If $m(\mathcal{C})=1$ then the partition $\{c(x): x \in \mathcal{C}\}$ of $\mathbb{T}^{3}$ by collapsed arcs of center manifolds is a measurable partition.

Proof. Consider $\left\{B_{i}\right\}_{i \in \mathbb{N}}$ a countable basis of open sets on $\mathbb{T}^{3}$. Then, we know that each point $x_{0} \in \mathbb{T}^{3}$ can be written as an intersection

$$
\left\{x_{0}\right\}=\bigcap B_{i}^{*}
$$

where $B_{i}^{*}=B_{i}$ or $B_{i}^{c}$. Thus,

$$
h^{-1}\left(\left\{x_{0}\right\}\right)=\bigcap h^{-1}\left(B_{i}^{*}\right)
$$

Since $h$ is continuous, $h^{-1}\left(B_{i}^{*}\right)$ is Lebesgue measurable (since it is an open or a closed set). The countable family $\left\{h^{-1}\left(B_{i}^{*}\right)\right\}$ separates the sets $h^{-1}\left(\left\{x_{0}\right\}\right)$. Thus $\mathcal{C}$ is a measurable partition. 
Even though the proof of the next lemma is simple, we remark that it is the key lemma to start the proof of the equivalence of Kolmogorov and Bernoulli properties in our setting. As we discussed before, an understanding of the behavior of volume measure along the center foliation is necessary. The next lemma relates the set of collapsed pieces with mono-atomicity of volume measure along center leaves. Intuitively speaking, it says that if $h$ is very good (the set of collapsed pieces has measure zero) then, for arbitrarily large compact sets we can control the distance of orbits of two points inside the same stable direction just comparing with the linear diffeomorphism. On the other hand, if $h$ is very bad (the set of collapsed pieces has full volume) then each center leaf can be seen as just one point! Thus, the direction of non-hyperbolic behavior (the center direction) does not disturbe the Bernoulli behavior generated by the hyperbolic directions, and this implies that $f$ is indeed Bernoulli.

Lemma 5.3.2 (Key Lemma). If

$$
m(\mathcal{C})=1
$$

then the disintegration along the center foliation is atomic with one atom per leaf.

Proof. By contradiction suppose that $\mathcal{C}$ has full volume measure and the disintegration is not atomic. Since $f$ and $f^{2}$ are Kolmogorov (in particular ergodic), we can assume without loss of generality that $f$ preserves the orientation of $\mathcal{F}^{c}$ (otherwise we work with $f^{2}$ ).

The segments from the family $\mathcal{C}$ form a measurable partition by Lemma 5.3.1. Let $\left\{m_{c(x)}\right\}_{c(x) \in \mathcal{C}}$ be the Rokhlin disintegration of volume on the partition $\mathcal{C}$, since $\mathcal{F}_{f}^{c}$ is not atomic, these conditional measures are not atomic on the leaf segment $c(x)$. Since the center foliation is orientable and $f$ preserves the orientation of $\mathcal{F}^{c}$, consider the following set $H_{\alpha}=\left\{y:\left[0_{x}, y\right] \subset c(x) \mid m_{c(x)}\left(\left[0_{x}, y\right]\right) \leq \alpha\right\}$, where $\left[0_{x}, y\right]$ is the segment from the inferior extreme $0_{x}$ of $c(x)$ to $y$. Because volume measure is invariant and

$$
f(c(x))=c(f(x))
$$


these conditional measures are invariant by $f$, i.e.,

$$
f_{*} m_{c(x)}=m_{c(f(x))}
$$

hence $H_{\alpha}$ is an invariant set. Notice that $m\left(H_{\alpha}\right) \leq \alpha$ and because we assume nonatomicity there must be an $\alpha \in(0,1)$ for which $0<m\left(H_{\alpha}\right) \leq \alpha$. By ergodicity we would have $\alpha \geq m\left(H_{\alpha}\right)=1$, absurd.

Thus, the disintegration is indeed atomic, and by Theorem B we conclude that we have exactly one atom per (global) leaf.

Remark 5.3.3. We remark that in the atomic case, even though each center leaf can be in a measure sense represented by one point, the situation is not the same as a 1-point extension of a Bernoulli transformation. This is because the leaves of the center foliation are non-compact, so we don't know exactly what is the space $\mathbb{T}^{3} / \mathcal{F}^{c}$, thus we cannot talk about a Bernoulli dynamics in a certain basis.

Definition 5.3.4. If $m(C)=0$ define

$$
X:=\mathbb{T}^{3} \backslash \mathcal{C}
$$

Otherwise, define

$$
X=\text { set of atoms. }
$$

\subsubsection{Partition by rectangles}

Take $\epsilon$ to be the partition of $\mathbb{T}^{3}$ by points. Let $\alpha$ be a partition of $\mathbb{T}^{3}$ by measurable sets such that the boundary of any element in $\alpha$ is piecewise smooth and such that each atom $D \in \alpha$ is an open set with boundary of zero measure. It is easy to construct such a partition on the 3-torus.

We will prove that $\alpha$ is VWB. Then we will take a sequence of such partitions $\alpha_{n}$ with: $\alpha_{1} \leq \alpha_{2} \leq \ldots$ such that $\alpha_{n} \rightarrow \epsilon$, concluding that $f$ is indeed Bernoulli by Theorems 5.1.4 and 5.1.5.

In order to do so, we follow the lines of Ornstein-Weiss [14] and Pesin [62] and we shall consider some specific partitions with dynamical meaning which will help 
us to control the behavior of $f^{i} \alpha, i \in \mathbb{N}$.

Given two points $y$ and $z$ close enough to each other, we know that $\mathcal{F}^{c s}(y)$ and $\mathcal{F}^{u}(z)$ will intersect each other and that the intersection is, locally, a single point. We denote this point by $[y, z]$. Sometimes along this section we also write $W^{c s}(y) \cap W^{u}(z)$ to mean the point $[y, z]$.

Definition 5.3.5. A measurable set $\Pi$ is called a $\delta$-rectangle at a point $w$ if $\Pi \subset$ $B(w, \delta)$ and for any $y, z \in \Pi$ the local intersection belongs to $\Pi$, that is

$$
[y, z] \in \Pi \text {. }
$$

The definition of rectangle here and in [62] is basically the same, the only difference being that in Pesin's case the measure is hyperbolic, so he works with stable foliation and we replace it by center-stable foliation. Also, since Pesin deals with non-hyperbolicity, he needs to work inside regular level sets (see [5] for definition), while do not need to do such restrictions here since we are working with absolutely partially hyperbolic diffeomorphisms.

Definition 5.3.6. Let $x \in \mathbb{T}^{3}$. The su-pseudoleaf at the point $x$ is the set

$$
\mathcal{F}^{s u}(x)=\bigcup_{y \in \mathcal{F}^{s}(x)} \mathcal{F}^{u}(x)
$$

Analogously, define the su-local pseudoleaf at $x$ as

$$
\mathcal{F}_{\text {loc }}^{s u}(x)=\bigcup_{y \in \mathcal{F}_{\text {loc }}^{s}(x)} \mathcal{F}_{\text {loc }}^{u}(x)
$$

The lift of a su-pseudo leaf at a point $x$ is a properly embedded topological hyperplane by Proposition 2.8 of [20], thus $\mathcal{F}^{s u}(x)$ is locally an embedded topological manifold.

Lemma 5.3.7. For any sufficiently small $\delta>0$, and any $x \in \mathbb{T}^{3}$ there is an open $\delta$-rectangle $R_{x}$ containing $x$.

Proof. Given $x \in \mathbb{T}^{3}$ and $\delta>0$. We can take $\delta_{2}>0$ small enough such that for any $y \in B\left(x, \delta_{2}\right)$ we have $[x, y],[y, x] \in B(x, \delta)$. Now take $\{a, b\}$ be the endpoints of the 
connected component of $\mathcal{F}^{u}(x) \cap \overline{B\left(x, \delta_{2}\right)}$ containing $x$.

Similarly, take $\{c, d\}$ to be the endpoints of the connected component of $\mathcal{F}^{s}(x) \cap$ $\overline{B\left(x, \delta_{2}\right)}$ containing $x$. Now, consider the region $\mathfrak{R}$ containing $x$, delimited by the leaves $\mathcal{F}_{\text {loc }}^{c s}(a), \mathcal{F}_{\text {loc }}^{c s}(b), \mathcal{F}_{\text {loc }}^{c u}(c)$ and $\mathcal{F}_{\text {loc }}^{c u}(d)$.

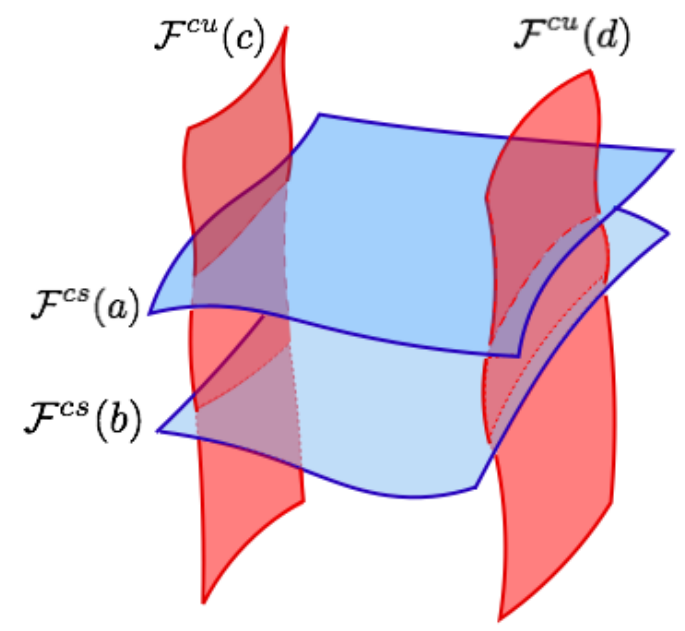

Figure 5.1: The "interior" region $\mathfrak{R}$ can be closed in a bounded region by adding two pseudoleaves to this figure.

To close this region in a rectangle format we need to add two "faces". Take $p, q \in \mathcal{F}_{\text {loc }}^{c s}(a) \cap B(x, \delta)$ and $q \in \mathcal{F}^{c}(p)$. Consider the pseudoleaves $\mathcal{F}_{\text {loc }}^{\text {su }}(p)$ and $\mathcal{F}_{\text {loc }}^{\text {su }}(q)$. These two pseudoleaves delimit a bounded open region $R$ inside $\mathfrak{R}$, and by a good choice of $q$ we can assume that $x \in R$. By taking $p, q$ close enough to $x$ we have that the diameter of $R$ is smaller then $\delta$ and it is easy to see that $R$ is indeed a rectangle.

Lemma 5.3.8. Given any $\delta>0$, one can find disjoint $\delta$-rectangles $\Pi_{1}, \ldots, \Pi_{m}$ each of them having dense interior and such that

$$
m\left(\mathbb{T}^{3} \backslash \bigcup_{i=1}^{m} \Pi_{i}\right)=0
$$

Proof. First of all, for each $x \in \mathbb{T}^{3}$ we construct a $\delta$-rectangle $R_{x}$, as done in Lemma 5.3.7 (that is, four faces are $c s$ and $c u$ leaves, and two faces are su-pseudoleaves) 
containing $x$ such that $R_{x}$ is open. Since $\mathbb{T}^{3}$ is compact we can take a finite number of these open rectangles $\left\{R_{1}, \ldots, R_{k}\right\}$ covering all the space.

Now, as done in [5], if $R_{i} \cap R_{j}$ is not empty, we can write this intersection as the union of five disjoint rectangles. Given $y \in R_{i} \cap R_{j}$, denote by $V^{u}(y)$ the connected component of $\mathcal{F}^{u}(y) \cap\left(R_{i} \cup R_{j}\right)$ containing $y$. If $R_{i} \cap R_{j} \neq \emptyset$ we can write $R_{i} \cup R_{j}$ as the union of:

$$
\begin{gathered}
\Pi_{1}:=R_{i} \cap R_{j}, \Pi_{2}=R_{i} \backslash \tilde{R}, \Pi_{3}=R_{j} \backslash \tilde{R}, \\
\Pi_{4}=\left(\tilde{R} \cap R_{i}\right) \backslash \Pi_{1} \text { and } \Pi_{5}=\left(\tilde{R} \cap R_{j}\right) \backslash \Pi_{1},
\end{gathered}
$$

where $\tilde{R}=\bigcup_{y \in R_{i} \cap R_{j}} \mathcal{V}^{u}(y)$. The proof that $\Pi_{i}$ is a rectangle for $1 \leq i \leq 5$ is identical to the one in Lemma 9.5.6 of [5].

It is easy to see that if $\Pi_{l}$ is not an open set for some $l=2, \ldots, 5$ then we can take $\operatorname{int}\left(\Pi_{l}\right)$ which is an open rectangle. Also, it is not hard to prove that $m\left(\partial \Pi_{l}\right)=0$ for $l=1,2,3,4,5$, because the boundaries are all small pieces of topological manifolds of dimension two. That is, $\left\{\Pi_{1}, \operatorname{int}\left(P_{2}\right), \operatorname{int}\left(P_{3}\right), \operatorname{int}\left(P_{4}\right), \operatorname{int}\left(P_{5}\right)\right\}$ are disjoint rectangles whose union is equal to $R_{i} \cup R_{j}$ modulo a zero measure set. This concludes the proof of the lemma.

Remark 5.3.9. In [62], Pesin didn't need to worry about the interior of the chosen rectangles, but in our case this subtle choice is crucial to overcome a possible absence of absolute continuity of the center foliation.

Definition 5.3.10. We say that a measurable set $A$ intersects a rectangle $\Pi$, leafwise if

$$
\mathcal{F}^{u}(w) \cap \Pi \subset A \cap \Pi, \text { for any } w \in A \cap \Pi \text {. }
$$

Lemma 5.3.11. If $E$ is a set intersecting a rectangle $\Pi$ leafwise then the intersection $E \cap \Pi$ is a rectangle.

Proof. Let $x, y \in E \cap \Pi$ and set $z=W^{u}(x) \cap W^{c s}(y)$. Since $x, y \in \Pi$ and $\Pi$ is a rectangle we have $z \in \Pi$. Now, because the intersection is leafwise we have

$$
z \in \mathcal{F}^{u}(x) \cap \Pi \subset \mathcal{F}^{u}(x) \cap E
$$


Lemma 5.3.12. If $\mathcal{C}$ is the set of segments of center manifolds that collapses under $h$, then given any point $x \in \mathbb{T}^{3} \backslash \mathcal{C}$ we have that

$$
\mathcal{F}^{u}(x) \subset \mathbb{T}^{3} \backslash \mathcal{C}
$$

In particular, given any rectangle $\Pi$, if $X=\mathbb{T}^{3} \backslash \mathcal{C}$ then $X$ intersects $\Pi$ leafwise.

Proof. Let $x \in \mathbb{T}^{3} \backslash \mathcal{C}$ and assume that we can find $y \in \mathcal{F}^{u}(x) \cap \mathcal{C}$. Then, there exists a closed segment $\gamma \subset \mathcal{F}^{c}(y)$ with $y \in \gamma$ and $\gamma \subset \mathcal{C}$. Take any $w \in \gamma \backslash\{y\}$ and consider $z=W^{u}(w) \cap W^{c}(x)$.

Since we assumed that the center Lyapunov exponent of the linearization $A$ of $f$ is positive, the semi-conjugacy $h$ sends strong unstable leaves of $f$ to unstable leaves of $A$. Note that stable leaves of $f$ are not necessarily mapped to stable leaves of $A$.

If $h(y)=h(w)$, then

$$
\mathcal{F}^{u}(h(x))=\mathcal{F}^{u}(h(y))=\mathcal{F}^{u}(h(w))=\mathcal{F}^{u}(h(z))
$$

Thus we conclude that $h(z) \in \mathcal{F}^{u}(h(x)) \cap \mathcal{F}^{c}(h(x))$. Since $W^{u}(h(x)) \cap W^{c}(h(x))=$ $\{h(x)\}$ we can take $z$ close enough to $y$ and then we have $h(x)=h(z)$, contradicting the hypothesis that $x \notin \mathcal{C}$.

Lemma 5.3.13. Given a rectangle $\Pi$ and $\beta>0$, one can find $N_{1}>0$ such that for any $N^{\prime} \geq N \geq N_{1}$ and $\beta$-almost every element $A \in \bigvee_{N}^{N^{\prime}} f^{k} \alpha$, there exists a subset $E \subset A$, intersecting $\Pi$ leafwise, for which:

$$
\frac{m(E)}{m(A)} \geq 1-\beta
$$

and E has non-empty interior.

Proof. The argument here is equal to Pesin's argument to prove Lemma 9.5.8 from [5], but since we want to prove non-empty interior (which is not pointed out in 
Pesin's proof) we repeat the argument. Given an element $D \in \alpha$ and $k>0$, denote by $B_{D}^{k} \subset f^{k}(D)$ the subset that intersects $\Pi$ leafwise.

Consider any point $x \in\left(f^{-k}(\Pi) \backslash f^{-k}\left(B_{D}^{k}\right)\right) \cap D$. Then we have for some constants $C>0$ and $0<a<1$ :

$$
\begin{aligned}
d(x, \partial D) \leq d(x, z) & =d\left(f^{k}\left(f^{-k}(x)\right), f^{k}\left(f^{-k}(z)\right)\right) \\
& \leq C a^{k} d\left(f^{-k}(x), f^{-k}(z)\right) \leq C \cdot \operatorname{diam}(\Pi) a^{k}
\end{aligned}
$$

Take $C_{1}=C \cdot \operatorname{diam}(\Pi)$, then

$$
d(x, \partial D) \leq C_{1} a^{k}
$$

Consequently, if we take

$$
C_{D}^{k}:=f^{k}\left\{x \in D \cap f^{-k}(\Pi): d(x, \partial D)>C_{1} a^{k}\right\}
$$

the set $C_{D}^{k} \subset f^{k}(D) \cap \Pi$ intersects $\Pi$ leafwise. Also $C_{D}^{k}$ is open.

Now, for any $N_{1}>0$ and $N^{\prime}>N_{1}$ set,

$$
G_{k}=\bigcup_{D \in \alpha}\left(f^{k}(D) \backslash C_{D}^{k}\right), G=\bigcup_{N_{1} \leq k \leq N^{\prime}} G_{k}
$$

Since the boundary of the set $D$ is piecewise smooth and has zero volume, it follows that

$$
m\left(G_{k}\right) \leq C_{2} a^{k}
$$

where $C_{2}>0$ is a constant. We now choose $N_{1}$ large enough to have

$$
m(G) \leq \sum_{k=N_{1}}^{\infty} m\left(G_{k}\right) \leq \beta^{2}
$$

Then, for $\beta$-almost every element $A \in \bigvee_{k=N}^{N^{\prime}} f^{k} \alpha$ we have that $m(A \cap G) \leq \beta \cdot m(A)$. It suffices to set

$$
E=A \cap G^{c} .
$$


Now,

$$
G^{c}=\bigcap_{N_{1} \leq k \leq N^{\prime}} \bigcap_{D \in \alpha}\left(f^{k}(D)^{c} \cup C_{D}^{k}\right)=\bigcap_{N_{1} \leq k \leq N^{\prime}} \bigcap_{D \in \alpha}\left(\operatorname{int}\left(f^{k}(D)^{c}\right) \cup \partial\left(f^{k}(D)^{c}\right) \cup C_{D}^{k}\right) .
$$

Since $D$ is an open set with zero measure boundary we have that $m\left(\partial f^{k}(D)^{c}\right)=0$, which implies

$$
m\left(G^{c}\right)=m\left(\bigcap_{N_{1} \leq k \leq N^{\prime}} \bigcap_{D \in \alpha}\left(\operatorname{int}\left(f^{k}(D)^{c}\right) \cup C_{D}^{k}\right)\right) .
$$

Since $C_{D}^{k}$ is open, the previous equality implies that $G^{c}$ is a set with dense interior. Thus, $E=A \cap G^{c} \subset A$ has dense interior as we wanted to show.

Lemma 5.3.14. Let $\Pi$ be a rectangle and $E \subset \Pi$ be a sub-rectangle intersecting $\Pi$ leafwise and with non-empty interior. Then, given any $x \in E$ we have

$$
m_{x}^{c s}\left(\mathcal{F}^{c s}(x) \cap E\right)>0 .
$$

Proof. Take any $x \in E$ and consider $y \in \operatorname{int}(E)$. Thus, we can take $r>0$ with $B_{r}(y) \subset E$. Consider the set $B_{r}^{c s}(y):=B_{r}(y) \cap \mathcal{F}^{c s}(y)$. Since $B_{r}^{c s}(y)$ is open in $\mathcal{F}^{c s}(y)$ we have $m_{y}^{c s}\left(B_{r}^{c s}(y)\right)>0$. Now, take the unstable holonomy $\pi_{y, x}^{u}$ and set $B^{c s}(x):=\pi^{u}\left(B_{r}^{c s}(y)\right)$. The unstable holonomy is a homeomorphism, thus we have $m_{x}^{c s}\left(B^{c s}(x)\right)>0$, and by the leafwise intersection we have that $B^{c s}(x) \subset E$, therefore

$$
m_{x}^{c s}\left(\mathcal{F}^{c s}(x) \cap E\right)>0
$$

\subsubsection{Construction of the function $\theta$}

We now proceed to the most important part of the proof: the construction of the function $\theta$ satisfying the hypothesis of Lemma 5.1.7. Such function $\theta$ should have the property that for most of the points $x$, the orbit of $x$ and of $\theta(x)$ has almost the same information (asymptotically) with respect to the partition in question. In 
particular, if we get good control on the distance $d\left(f^{n}(x), f^{n}(\theta(x))\right)$ we can expect that for most points $x, f^{n}(x)$ and $f^{n}(\theta(x))$ belong to the same partition element; this will be much clearer below. In order to get this control, we need to restrict to a compact set (where $h$ is uniformly continuous) and then use the fact that the center manifold of $A$ is uniformly contracting and $h$ sends center manifolds to center manifolds. This is what we do in the next lemma.

Lemma 5.3.15. Given any $\varepsilon>0$ and any compact set $K \subset X$ (see definition 5.3.4) there exists $n_{0} \in \mathbb{N}$ such that for any two points $a \in K, b \in \mathcal{F}^{c s}(a) \cap K$, with $d(a, b)<\frac{1}{2}$ we have

$$
d\left(f^{n}(a), f^{n}(b)\right)<\varepsilon, \text { whenever } f^{n}(a), f^{n}(b) \in K \text { and } n \geq n_{0} .
$$

Proof. We split the proof in two cases.

First case: $X=\mathbb{T}^{3} \backslash \mathcal{C}$.

Consider the lifts to the universal cover $\tilde{A}, \tilde{f}: \mathbb{R}^{3} \rightarrow \mathbb{R}^{3}$ and $\tilde{h}: \mathbb{R}^{3} \rightarrow \mathbb{R}^{3}$ the lift of the conjugacy, such that $\tilde{h}(0)=0$. We know that

$$
\tilde{A}^{n} \circ \tilde{h}=\tilde{h} \circ \tilde{f}^{n}
$$

for all $n$. Thus

$$
\left(e^{\lambda_{A}^{c}}\right)^{n} d(\tilde{h}(a), \tilde{h}(b)) \geq d\left(\tilde{A}^{n} \circ \tilde{h}(a), \tilde{A}^{n} \circ \tilde{h}(b)\right)=d\left(\tilde{h} \circ \tilde{f}^{n}(a), \tilde{h} \circ \tilde{f}^{n}(b)\right) .
$$

Since $h$ is a bounded distance from the identity, then if $d(a, b)<\delta$ we have:

$$
d\left(\tilde{A}^{n} \circ \tilde{h}(a), \tilde{A}^{n} \circ \tilde{h}(b)\right) \leq\left(e^{\lambda_{A}^{c}}\right)^{n} \cdot D
$$

for a certain constant $D>0$. By the uniform continuity of $h^{-1}$ inside $h(K)$ we can take $n_{0}$ big enough so that $n \geq n_{0}$ implies:

$$
d\left(f^{n}(a), f^{n}(b)\right)<\varepsilon
$$


Second case: $X=$ set of atoms.

In this case, we know by Lemma 5.3.2 that each leaf has only one atom, that is, for almost every $x \in \mathbb{T}^{3}$ we have $X \cap \mathcal{F}^{c}(x)=\left\{a_{x}\right\}$. Thus, given any two points $a, b \in X, a$ and $b$ are not collapsed by $h$ (since they do not belong to the same central leaf). Thus the proof of the first case works for this case as well.

Lemma 5.3.16. For any $\delta>0$, there exists $0<\delta_{1}<\delta$ with the following property. Let $\Pi$ be a $\delta_{1}$-rectangle and $E$ a set intersecting $\Pi$ leafwise and such that $E \cap \Pi$ has non-empty interior. Then we can construct a bijective function $\theta: E \cap \Pi \rightarrow \Pi$ such that

$$
|\operatorname{Jac}(\theta)(y)-1| \leq \delta
$$

and such that for every $x \in E \cap \Pi$ we have

$$
\theta(x) \in \mathcal{F}^{c s}(x)
$$

Proof. Given $\delta>0$, by Theorem 2.2.10 we can take $\delta_{1}>0$ small enough so that if $d\left(w, w_{1}\right)<\delta_{1}$ then the holonomy map $\pi^{u}=\pi_{w, w_{1}}^{u}: W^{c s}(w) \rightarrow W^{c s}\left(w_{1}\right)$ satisfies

$$
\left|\operatorname{Jac}\left(\pi^{u}\right)(x)-1\right| \leq \frac{1}{3} \delta
$$

Since $E$ intersects $\Pi$ leafwise by Lemma 5.3 .11 we know that $E \cap \Pi$ is a sub-rectangle, and since it has non-empty interior we conclude by Lemma 5.3.14 that for $x \in \Pi$,

$$
m_{x}^{c s}\left(\mathcal{F}^{c s}(x) \cap E \cap \Pi\right)>0 .
$$

Because $\mathcal{F}^{c s}(x) \cap E \cap \Pi$ and $\mathcal{F}^{c s}(x) \cap \Pi$ are both Lebesgue spaces (with the normalized measures) we can construct a bijection $\theta_{0}: \mathcal{F}^{c s}(x) \cap E \cap \Pi \rightarrow \mathcal{F}^{c s}(x) \cap \Pi$ preserving the normalized measures.

Now, given any $y \in E \cap \Pi$ we define (using that the intersection is leafwise and inside the rectangle)

$$
\theta(y):=\left(\pi_{y, x}^{u}\right)^{-1} \circ \theta_{0} \circ \pi_{y, x}^{u}(y)
$$




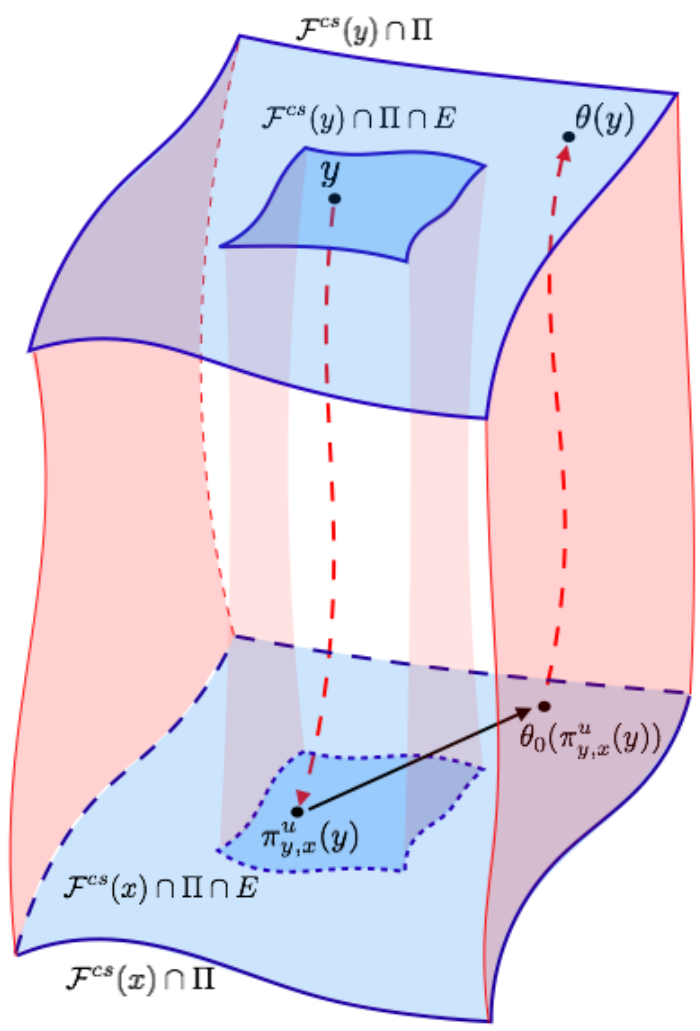

Figure 5.2: The larger rectangle is $\Pi$ and the smaller one is $E \cap \Pi$. The red lines represent unstable manifolds.

This $\theta: E \cap \Pi \rightarrow \Pi$ is well defined and $\theta(y) \in \mathcal{F}^{c s}(y) \cap \Pi$. Furthermore, by (5.2) we have

$$
|\operatorname{Jac}(\theta)(y)-1| \leq \delta
$$

as we wanted.

The proof of the following lemma is equal to the proof of Lemma 9.5.10 of Barreira-Pesin ( [5, pg.295]), with the small difference that in Pesin's case $\theta(y) \in$ $\mathcal{F}^{s}(y) \cap E$, but in our case $\theta(y) \in \mathcal{F}^{c s}(y) \cap E$. For the sake of completeness we reproduce the proof here.

Lemma 5.3.17. For any $0<\varepsilon^{\prime} \leq \varepsilon$, one can find $N_{2}>0$ such that for any $N^{\prime} \geq N \geq N_{2}$ and E-almost every element $A \in \bigvee_{k=N}^{N^{\prime}} f^{k} \alpha$, there exist a subset $E \subset A$ and a bijective and onto map $\theta: E \rightarrow M$ for which 
1.

$$
\frac{m(E)}{m(A)} \geq 1-2 \varepsilon
$$

2. the map $\theta$ is $23 \varepsilon$-measure preserving;

3. for any $y \in E$,

$$
\theta(y) \in \mathcal{F}^{c s}(y) \cap E
$$

Proof. Given $\varepsilon^{\prime}>0$, choose $\delta_{1}>0$ (and $\delta_{1}<\varepsilon$ ) as in Lemma 5.3.16. Let $\eta=$ $\left\{\Pi_{1}, \ldots, \Pi_{b}\right\}$ be a partition of $\mathbb{T}^{3}$ where $\Pi_{1}, \ldots, \Pi_{b}$ are open and disjoint $\delta_{1}$-rectangles constructed in Lemma 5.3.8 . Set

$$
\delta:=\varepsilon \cdot \min \left\{m\left(\Pi_{i}\right): 1 \leq i \leq b\right\}
$$

By Lemma 5.2.1 we can take $N_{0}>0$ such that for any $N^{\prime}>N \geq N_{0}$ and $\delta$-almost every element $A \in \bigvee_{k=N}^{N^{\prime}} f^{k} \alpha$ we have

$$
\left|\frac{m\left(A \cap \Pi_{i}\right)}{m(A)}-m\left(\Pi_{i}\right)\right| \leq \delta,
$$

for all $1 \leq i \leq b$.

Take

$$
\beta:=b^{-1} \varepsilon \min _{1 \leq i \leq b}\left\{m\left(\Pi_{i}\right)\right\} .
$$

For each rectangle $\Pi_{i}$, by Lemma 5.3.13, $\exists N_{1 i}>0$ such that $\forall N^{\prime} \geq N \geq N_{1 i}$ and $\beta$-almost every $A \in \bigvee_{k=N}^{N^{\prime}} f^{k} \alpha, \exists F_{i} \subset A$ intersecting $\Pi_{i}$ leafwise such that

$$
\frac{m\left(F_{i}\right)}{m(A)} \geq 1-\beta
$$

Set $N_{2}:=\max \left\{N_{0}, \max _{1 \leq i \leq m} N_{1 i}\right\}$. It follows that for $\varepsilon$-almost every $A \in \bigvee_{k=N}^{N^{\prime}} f^{k} \alpha$, there exists a subset

$$
E:=\bigcup_{1 \leq i \leq b}\left(F_{i} \cap \Pi_{i}\right) \subset A
$$


such that $E$ intersects each $\Pi_{i}$ leafwise, $1 \leq i \leq b$. Furthermore, note that

$$
\frac{m\left(F_{i}\right)}{m(A)} \leq \frac{m\left(F_{i} \cap \Pi_{i}\right)}{m(A)}+\frac{m\left(A \cap \Pi_{i}^{c}\right)}{m(A)}=\frac{m\left(F_{i} \cap \Pi_{i}\right)}{m(A)}+1-\frac{m\left(A \cap \Pi_{i}\right)}{m(A)}
$$

Thus,

$$
\begin{aligned}
\frac{m(E)}{m(A)}=\frac{\sum_{i=1}^{b} m\left(F_{i} \cap \Pi_{i}\right)}{m(A)} & \geq \sum_{i=1}^{b}\left(\frac{m\left(F_{i}\right)}{m(A)}-1+\frac{m\left(A \cap \Pi_{i}\right)}{m(A)}\right) \\
& \geq b(1-\beta)-b+1=1-b \beta>1-2 \varepsilon
\end{aligned}
$$

Claim 1: $\quad m\left((A \backslash E) \cap \Pi_{i}\right) \leq \varepsilon \cdot m(E) \cdot m\left(\Pi_{i}\right) \cdot(1+3 \varepsilon)$. Proof: Indeed since $m(E) / m(A) \geq 1-\beta$ then $m\left(A \cap E^{c}\right) \leq \beta m(A)$. Thus, for any $1 \leq i \leq b$ we have

$$
\begin{aligned}
m\left(A \cap E^{c} \cap \Pi_{i}\right) \leq m\left(A \cap E^{c}\right) & \leq \beta \cdot m(A) \leq b^{-1} \cdot \varepsilon \cdot m(A) m\left(\Pi_{i}\right) \\
& \leq \frac{\varepsilon}{b(1-\beta)} \cdot m(E) m\left(\Pi_{i}\right) \\
& \leq \varepsilon(1+3 \varepsilon) \cdot m(E) m\left(\Pi_{i}\right) .
\end{aligned}
$$

Consider $E_{i}:=F_{i} \cap \Pi_{i}=E \cap \Pi_{i}$.

\section{Claim 2:}

$$
\left|\frac{m\left(E_{i}\right)}{m(E)}-m\left(\Pi_{i}\right)\right| \leq 11 \cdot \varepsilon \cdot m\left(\Pi_{i}\right)
$$

Proof: First of all, we write

$$
\begin{aligned}
\left|\frac{m\left(E_{i}\right)}{m(E)}-m\left(\Pi_{i}\right)\right| & =\left|\frac{m(A)}{m(E)} \cdot \frac{m\left(A \cap \Pi_{i}\right)}{m(A)}-\frac{m\left(A \cap E^{c} \cap \Pi_{i}\right)}{m(E)}-m\left(\Pi_{i}\right)\right| \\
& =\left|\frac{m(A)}{m(E)} \cdot\left[\frac{m\left(A \cap \Pi_{i}\right)}{m(A)}-m\left(\Pi_{i}\right)\right]-\frac{m\left(A \cap E^{c} \cap \Pi_{i}\right)}{m(E)}+\left[\frac{m(A)}{m(E)}-1\right] \cdot m\left(\Pi_{i}\right)\right|
\end{aligned}
$$

Recall that

$$
\begin{gathered}
m(E) / m(A)>1-2 \varepsilon, \\
\delta \leq \varepsilon \cdot m\left(\Pi_{i}\right)
\end{gathered}
$$


and that by the first claim we have

$$
\frac{m\left((A \backslash E) \cap \Pi_{i}\right)}{m(E)} \leq \varepsilon \cdot m\left(\Pi_{i}\right) \cdot(1+3 \varepsilon) .
$$

Thus, by (5.3), applying the triangular inequality to the last equation we obtain

$$
\begin{aligned}
\left|\frac{m\left(E_{i}\right)}{m(E)}-m\left(\Pi_{i}\right)\right| & \leq\left|\frac{m(A)}{m(E)}\right|\left|\frac{m\left(A \cap \Pi_{i}\right)}{m(A)}-m\left(\Pi_{i}\right)\right|+\left|\frac{m\left(A \cap E^{c} \cap \Pi_{i}\right)}{m(E)}\right|+\left|\frac{m(A)}{m(E)}-1\right| m\left(\Pi_{i}\right) \\
& \leq \frac{\varepsilon}{1-2 \varepsilon} \cdot m\left(\Pi_{i}\right)+\varepsilon(1+3 \varepsilon) m\left(\Pi_{i}\right)+\frac{2 \varepsilon}{1-2 \varepsilon} \cdot m\left(\Pi_{i}\right) \\
& =\left(\frac{3 \varepsilon}{1-2 \varepsilon}+\varepsilon(1+3 \varepsilon)\right) \cdot m\left(\Pi_{i}\right)
\end{aligned}
$$

For $\varepsilon<1 / 3$ we have

$$
\frac{3 \varepsilon}{1-2 \varepsilon}+\varepsilon(1+3 \varepsilon) \leq 11 \varepsilon
$$

which implies

$$
\left|\frac{m\left(E_{i}\right)}{m(E)}-m\left(\Pi_{i}\right)\right| \leq 11 \varepsilon \cdot m\left(\Pi_{i}\right),
$$

as we wanted to show.

Let $\theta_{i}: E \cap \Pi_{i} \rightarrow \Pi_{i}, i=1, \ldots, b$ be the bijective maps constructed in Lemma 5.3.16. Define the map

$$
\theta: E \rightarrow M, \theta(y)=\theta_{i}(y) \text { if } y \in E_{i}
$$

Because the Jacobian of $\theta_{i}$ is controlled, for a measurable set $B \subset E$ we have:

$$
\left|\frac{m\left(\theta_{i}\left(B_{i}\right)\right)}{m\left(\Pi_{i}\right)} \cdot \frac{m\left(E_{i}\right)}{m\left(B_{i}\right)}-1\right| \leq \varepsilon^{\prime},
$$

where $B_{i}:=B \cap \Pi_{i}$. Then,

$$
\begin{gathered}
\left|\frac{m\left(\theta_{i}\left(B_{i}\right)\right) m(E)}{m\left(B_{i}\right)}-1\right| \leq\left|\frac{m\left(\theta_{i}\left(B_{i}\right)\right) m\left(E_{i}\right)}{m\left(B_{i}\right) m\left(\Pi_{i}\right)}-1\right|+\left|\frac{m\left(\theta_{i}\left(B_{i}\right)\right) m\left(E_{i}\right)}{m\left(B_{i}\right) m\left(\Pi_{i}\right)} \cdot\left(\frac{m(E) m\left(\Pi_{i}\right)}{m\left(E_{i}\right)}-1\right)\right| \leq \\
\leq \varepsilon^{\prime}+\left(1+\varepsilon^{\prime}\right)\left|\frac{m(E) m\left(\Pi_{i}\right)}{m\left(E_{i}\right)}-1\right|
\end{gathered}
$$


Note that, for $\varepsilon<1 / 22$,

$$
\begin{gathered}
\left|1-\frac{m\left(\Pi_{i}\right) m(E)}{m\left(E_{i}\right)}\right| \leq \frac{11 \varepsilon m\left(\Pi_{i}\right) m(E)}{m\left(E_{i}\right)} \Rightarrow \\
-\frac{11 \varepsilon}{1+11 \varepsilon} \leq \frac{m\left(\Pi_{i}\right) m(E)}{m\left(E_{i}\right)}-1 \leq \frac{11 \varepsilon}{1-11 \varepsilon} \Rightarrow\left|1-\frac{m(E) m\left(\Pi_{i}\right)}{m\left(E_{i}\right)}\right| \leq \frac{11 \varepsilon}{1-11 \varepsilon} .
\end{gathered}
$$

Thus

$$
\left|\frac{m\left(\theta_{i}\left(B_{i}\right)\right) m(E)}{m\left(B_{i}\right)}-1\right| \leq \varepsilon^{\prime}+\left(1+\varepsilon^{\prime}\right) \frac{11 \varepsilon}{1-11 \varepsilon} \leq \varepsilon^{\prime}+\left(1+\varepsilon^{\prime}\right) 22 \varepsilon \leq 23 \varepsilon
$$

Finally, observe that

$$
\left|\frac{m(\theta(B)) m(E)}{m(B)}-1\right|=\left|\frac{\sum_{i=1}^{b} m\left(\theta_{i}\left(B_{i}\right)\right) m(E)}{m(B)}-1\right|=\left|\sum_{i=1}^{b}\left[\frac{m\left(\theta_{i}\left(B_{i}\right)\right) m(E)}{m\left(B_{i}\right)}-1\right] \cdot \frac{m\left(B_{i}\right)}{m(B)}\right| .
$$

Then, by (5.4) we have

$$
\left|\frac{m(\theta(B)) m(E)}{m(B)}-1\right| \leq \sum_{i=1}^{b} 23 \cdot \varepsilon \cdot \frac{m\left(B_{i}\right)}{m(B)}=23 \cdot \varepsilon
$$

concluding that $\theta$ is indeed $23 \cdot \varepsilon$-measure preserving. The third item is an immediate consequence of the construction of $\theta$.

\subsubsection{Conclusion of the proof of Theorem A}

Lemma 5.3.18. The partition $\alpha$ is VWB.

Proof. Fix $\varepsilon>0,0<\varepsilon^{\prime}<\varepsilon$ and choose $N_{2}$ as in Lemma 5.3.17 and $Y$ the exception set obtained in Lemma 5.3.17, that is,

$$
m(Y) \leq 23 \varepsilon, Y=\text { union of some elements of } \bigvee_{k=N}^{N^{\prime}} f^{k} \alpha,\left(N^{\prime}>N \geq N_{2}\right)
$$

Consider $A \cap Y=\emptyset, A \in \bigvee_{k=N}^{N^{\prime}} f^{k} \alpha$ and consider $E \subset A$ and $\theta: E \rightarrow M$ constructed in the previous lemma. Take the sequence of partitions $\left\{\xi_{i}\right\}_{1}^{n}=\left\{f^{-i} \alpha\right\}_{1}^{n}$ 
and $\left\{\eta_{i}\right\}_{1}^{n}=\left\{f^{-i} \alpha \mid A\right\}_{1}^{n}$, that is, each $\xi_{i}$ is a partition of $M$ and each $\eta_{i}$ is a partition of $A$. Now take $\bar{\theta}: A \rightarrow M$ given by

$$
\bar{\theta}(x):=\theta(x), \text { for } x \in E
$$

and

$$
\bar{\theta}(x)=x_{0} \text { for } x \in A \backslash E, \quad(m(A \backslash E)<2 \varepsilon),
$$

where $x_{0}$ is an arbitrary point.

Take an arbitrary $\zeta<1$ and consider $K \subset E \cap X$ (see definition 5.3.4) a compact set with

$$
m(K)>\zeta \cdot m(E)
$$

Take $\kappa$ the set of points of $K$ such that the past and future Birkhoff averages coincide and converge to $m(K)$, that is: $x \in \kappa$ if $x \in K$ and

$$
\lim _{n \rightarrow-\infty} \frac{1}{|n|} \sum_{j=0}^{n-1} \chi_{K}\left(f^{j}(x)\right)=\lim _{n \rightarrow \infty} \frac{1}{n} \sum_{j=0}^{n-1} \chi_{K}\left(f^{j}(x)\right)=m(K)
$$

By Birkhoff's Theorem we know that $m(\kappa)=m(K)$. Take $F:=\kappa \cap \theta^{-1}(\kappa)$. Since $\theta$ is $23 \varepsilon$-measure preservig, taking $\zeta$ close enough to one we have that

$$
m(E \backslash F) \leq 20 \cdot \varepsilon
$$

Now, observe that by Lemma 5.3 .15 we can take $n_{0} \geq 0$ such that for any $x \in \kappa$ we have

$$
d\left(f^{n}(x), f^{n}(\theta(x))\right)<\varepsilon^{\prime}
$$

for all $n \geq n_{0}$ with $f^{n}(x), f^{n}(\theta(x)) \in \kappa$.

Now we observe that if $x \in \kappa, i \geq n_{0}$ and $e\left(l_{i}(x)-m_{i}(\theta(x))\right)=1$ then either

- $f^{i}(x) \notin \kappa$ or $f^{i}(\theta(x)) \notin \kappa ;$ or 
- $d\left(f^{i}(x), f^{i}(\theta(x))\right)<\varepsilon^{\prime}$ and then,

$$
d\left(f^{i}(x), \partial A_{l_{i}(x)}\right)<\varepsilon^{\prime} \Rightarrow f^{i}(x) \in O_{\varepsilon^{\prime}}\left(A_{l_{i}(x)}\right) .
$$

Set $O_{\varepsilon^{\prime}}:=\bigcup_{i=1}^{k} O_{\varepsilon^{\prime}}\left(A_{i}\right)$ and call $J=\left\{j \in \mathbb{N}\right.$ such that $f^{j}(x) \notin \kappa$ or $f^{j}(\theta(x)) \notin$ $\kappa\}, J_{n}:=J \cap[1, n]$.

By the definition of the function $e$ we have

$$
\frac{1}{n} \sum_{i=1}^{n} e\left(l_{i}(x)-m_{i}(\theta(x))\right) \leq \frac{1}{n} \sum_{j=1}^{n} \chi_{O_{\varepsilon^{\prime}}}\left(f^{j}(x)\right)+\frac{1}{n} \# J_{n}
$$

By ergodicity the right side converges to $\left[m\left(O_{\varepsilon^{\prime}}\right)+\operatorname{dens}(J)\right]$ for almost every $x$. Thus, it follows from Lemma 5.1.7 that

$$
d\left(\left\{\xi_{i}\right\}_{1}^{n},\left\{\eta_{i}\right\}_{1}^{n}\right) \leq 10000 \varepsilon
$$

Since $\varepsilon>0$ is arbitrary it follows that $\alpha$ is VWB.

Corollary 5.3.19. $f$ is Bernoulli. 


\section{Bibliography}

[1] T. Adams. Smorodinsky's conjecture on rank one mixing. Proc. Amer. Math. Soc., 126:739-744, 1998.

[2] A. Avila and M. Viana. Extremal lyapunov exponents: an invariance principle and applications. Inventiones Mathematicae, 181:115-174, 2010.

[3] A. Avila, M. Viana, and A. Wilkinson. Absolute continuity, lyapunov exponents and rigidity i: geodesic flows. Journal of European Math. Soc., to appear.

[4] A. Baraviera and C. Bonatti. Removing zero lyapunov exponents. Ergodic Theory and Dynamical Systems, pages 1655-1670, 2003.

[5] L. Barreira and Y. Pesin. Dynamics of Systems with Nonzero Lyapunov Exponents. Cambridge University Press, 2007.

[6] C. Bonatti and A. Wilkinson. Transitive partially hyperbolic diffeomorphisms on 3-manifolds. Topology, 2005.

[7] M. Brin, D. Burago, and D. Ivanov. On partially hyperbolic diffeomorphisms of 3-manifolds with commutative fundamental group. Modern dynamical systems and applications, pages 307-312, 2004.

[8] M. Brin, D. Burago, and D. Ivanov. Dynamical coherence of partially hyperbolic diffeomorphisms of the 3-torus. J. Mod. Dyn, pages 1-11, 2009.

[9] M. Brin and Y. Pesin. Partially hyperbolic dynamical systems. Math. USSRIzv., (8):177-218, 1974.

[10] K. Burns, C. Pugh, M. Shub, and A. Wilkinson. Recent results about stable ergodicity. Proc. Symposia A.M.S., pages 327-366, 2001. 
[11] K. Burns, C. Pugh, and A. Wilkinson. Stable ergodicity and anosov flows. Topology, 39(149-159), 2000.

[12] K. Burns and A. Wilkinson. On the ergodicity of partially hyperbolic systems. Annals of Mathematics, 171(1):451-489, 2010.

[13] D. Dolgopyat. On mixing properties of compact group extensions of hyperbolic systems. Israel Journal of Mathematics, 130(157-205), 2002.

[14] D.Ornstein and B.Weiss. Geodesic flows are bernoullian. Israel Journal of Mathematics, 14(2):Hebrew University Magnes Press-198, 1973.

[15] Manfred Einsiedler and Thomas Ward. Ergodic theory with a view towards number theory, volume 259 of Graduate Texts in Mathematics. Springer-Verlag London, Ltd., London, 2011.

[16] J. Franks. Anosov diffeomorphisms. Global Analysis (Proc. Sympos. Pure Math., Vol. XIV, Berkeley, Calif., 1968) pp. 61-93 Amer. Math. Soc., 1970.

[17] G. Gallavotti and D. Ornstein. Billiards and bernoulli schemes. Comm. Math. Phys., 38:83-101, 1974.

[18] A. Gogolev. How typical are pathological foliations in partially hyperbolic dynamics: an example. Israel Journal of Mathematics, 187(1):493-502, 2012.

[19] A. Hammerlindl. Leaf conjugacies on the torus. Ergodic Theory and Dynamical Systems, to appear, 2009.

[20] A. Hammerlindl. Leaf conjugacies on the torus. PhD Thesis, University of Toronto, 2009.

[21] A. Hammerlindl and R. Potrie. Pointwise partial hyperbolicity in 3-dimensional nilmanifolds. Journal of the London Mathematical Society, to appear.

[22] A. Hammerlindl and R. Ures. Ergodicity and partial hyperbolicity on the 3torus. Communications in Contemporary Mathematics, to appear.

[23] B. Hasselblatt. Problems in dynamical systems and related topics raised in connection with the clay mathematics institute/mathematical sciences research institute workshop on "recent progress in dynamics". 
[24] F. Rodriguez Hertz, M. A. Rodriguez Hertz, and R. Ures. A survey about partially hyperbolic dynamics. Fields Institute Communications, 51:35-88, 2007.

[25] F. Rodriguez Hertz, M. A. Rodriguez Hertz, and R. Ures. Accessibility and stable ergodicity for partially hyperbolic diffeomorphisms with 1d-center bundle. Inventiones Mathematicae, 172:353-381, 2008.

[26] F. Rodriguez Hertz, M. A. Rodriguez Hertz, and R. Ures. A non-dynamically coherent example in $t^{3}$. in preparation, 2009.

[27] M. Hirayama and Y. Pesin. Non-absolutely continuous foliations. Israel Journal of Mathematics, 160:173-187, 2007.

[28] S. Kalikow. T,t-1 transformation is not loosely bernoulli. Annals of Mathematics, 115(2):393-409, 1982.

[29] S. Kalikow and R. Mccutcheon. An Outline of Ergodic Theory. Cambridge University Press, 2010.

[30] A. Katok. Smooth non-bernoulli k-automorphisms. Inventiones Mathematicae, 61:291-300, 1980 .

[31] A. Katok and B. Hasselblatt. Introduction to the modern theory of dynamical systems. Cambridge University Press, 1999.

[32] A. N. Kolmogorov. A new metric invariant of transient dynamical systems and automorphisms in Lebesgue spaces. Dokl. Akad. Nauk SSSR (N.S.), 119:861864, 1958.

[33] F. Ledrappier and L. S. Young. The metric entropy of diffeomorphisms: Part i: Characterization of measures satisfying pesin's entropy formula. Annals of Mathematics, 122(3):509-539, 1985.

[34] A. Manning. There are no new anosov diffeomorphisms on tori. American Journal of Mathematics, 96(3):422-429, 1974.

[35] D. Meyer and F. Strömberg. Symbolic dynamics for the geodesic flow on hecke surfaces. Journal of Modern Dynamics, 2(4):581-627, 2008.

[36] F. Micena and A. Tahzibi. Regularity of foliations and lyapunov exponents for partially hyperbolic dynamics. Nonlinearity, 23:1071-1082, 2013. 
[37] J. Milnor. Fubini foiled: Katok's paradoxical example in measure theory. The Mathematical Intelligencer, 19(2):30-32, 1997.

[38] D. Ornstein. Bernoulli shifts with the same entropy are isomorphic. Advances in Math., (4):337-352, 1970.

[39] D. Ornstein. Imbedding bernoulli shifts in flows, contributions to ergodic theory and probability. Lecture Notes in Math, Springer Berlin, pages 178-218, 1970.

[40] D. Ornstein. Two bernoulli shifts with infinite entropy are isomorphic. Advances in Mathematics, (5):339-348, 1970.

[41] D. Ornstein. A kolmogorov automorphism that is not a bernoulli. Advances in Mathematics, 10:49-62, 1973.

[42] D. Ornstein and B. Weiss. Statistical properties of chaotic systems. Bulletin of the American Mathematical Society, 24(1), 1991.

[43] D. Ornstein and B. Weiss. On the bernoulli nature of systems with some hyperbolic structure. Ergodic Theory and Dynamical Systems, 18(441-456), 1998.

[44] G. Ponce and A. Tahzibi. Central lyapunov exponents of partially hyperbolic diffeomorphisms on $\mathbb{T}^{3}$. Proc. Amer. Math. Soc., 142:3193-3205, 2014.

[45] G. Ponce, A. Tahzibi, and R. Varão. Minimal yet measurable foliations. Journal of Modern Dynamics, 8(1):93-107, 2014.

[46] G. Ponce, A. Tahzibi, and R. Varão. Bernoulli property for partially hyperbolic diffeomorphisms on the 3-torus. in preparation.

[47] C. Pugh and M. Shub. Ergodic attractors. Trans. Amer. Math. Soc., 312(1):154, 1989.

[48] C. Pugh and M. Shub. Stably ergodic dynamical systems and partial hyperbolicity. J. Complexity, 13(1):125-179, 1997.

[49] M. Ratner. Anosov flows with gibbs measures are also bernoullian. Israel Journal of Mathematics, 17:380-391, 1974. 
[50] M. Ratner. Bernoulli flows over maps of the interval. Israel Journal of Mathematics, 31:380-391, 1978.

[51] V. A. Rohlin. Lectures on the entropy theory of transformations with invariant measure. Uspehi Mat. Nauk, $22(5$ (137)):3-56, 1967.

[52] V. A. Rohlin and Y. Sinai. Construction and properties of invariant measurable partitions. Doklady Akademii Nauk SSSR, 141:pp. 1038-1041, 1961 (Russian); Soviet Mathematics, vol. 2, pp. 1611-1614, 1961 (English)., 1971.

[53] D. J. Rudolph. Classifying the isometric extensions of a bernoulli shift. Journal d'Analyse Mathématique, 34(36-60), 1978.

[54] D. J. Rudolph. If a two-point extension of a bernoulli shift has an ergodic square, then it is bernoulli. Israel Journal of Mathematics, 30(159-180), 1978.

[55] D. Ruelle and A. Wilkinson. Absolutely singular dynamical foliations. Comm. Math. Phys., 219:481-487, 2001.

[56] R. Saghin and Z. Xia. Geometric expansion, Lyapunov exponents and foliations. Ann. Inst. H. Poincaré Anal. Non Linéaire, 26(2):689-704, 2009.

[57] M. Shub and A. Wilkinson. Pathological foliations and removable zero exponents. Inventiones Mathematicae, 2000.

[58] D. Sullivan. A counterexample to the periodic orbit conjecture. Inst. Hautes Études Sci. Publ. Math., (46):5-14, 1976.

[59] R. Ures. Intrinsic ergodicity of partially hyperbolic diffeomorphisms with a hyperbolic linear part. Proc. Amer. Math. Soc., 140(6), 2012.

[60] R. Varão. Center foliation: absolute continuity, disintegration and rigidity. Ergodic Theory and Dynamical Systems, to appear.

[61] Y.Katznelson. Ergodic automorphisms of $t^{n}$ are bernoulli shifts. Israel Journal of Mathematics, 10(186-195), 1971.

[62] Y.Pesin. Characteristic lyapunov exponents, and smooth ergodic theory. Russ. Math. Surv., 32:55-114, 1977. 
[63] Y.Pesin. A description of the $\pi$-partition of a diffeomorphism with an invariant measure. Math. Notes, 22:506-515, 1977.

[64] Y.Pesin. Lectures on partial hyperbolicity and stable ergodicity. Zurich Lectures in Advanced Mathematics, 2004. 